\title{
Dynamic Pricing and Matching for Two-Sided Queues
}

\author{
Sushil Mahavir Varma, Pornpawee Bumpensanti, Siva Theja Maguluri, He Wang \\ School of Industrial and Systems Engineering, Georgia Institute of Technology, Atlanta, GA 30332 \\ sushil@gatech.edu, pornpawee@gatech.edu, siva.theja@gatech.edu, he.wang@isye.gatech.edu
}

\begin{abstract}
Motivated by diverse applications in sharing economy and online marketplaces, we consider optimal pricing and matching control in a two-sided queueing system. We assume that heterogeneous customers and servers arrive to the system with price-dependent arrival rates. The compatibility between servers and customers is specified by a bipartite graph. Once a pair of customer and server are matched, they depart from the system instantaneously. The objective is to maximize long-run average profits of the system while minimizing average waiting time. We first propose a static pricing and max-weight matching policy, which achieves $O(\sqrt{\eta})$ optimality rate when all of the arrival rates are scaled by $\eta$. We further show that a dynamic pricing and modified max-weight matching policy achieves an improved $O\left(\eta^{1 / 3}\right)$ optimality rate. Under a broad class of pricing policies, we prove that any matching policy has an optimality rate that is lower bounded by $\Omega\left(\eta^{1 / 3}\right)$. Thus, the dynamic pricing policy and modified max-weight matching policy achieves the optimal rate. In addition, we propose a constraint generation algorithm that solves an approximation of the MDP and demonstrate strong numerical performance of this algorithm.
\end{abstract}

Key words: queueing, dynamic pricing, max-weight matching, Markov decision process

\section{Introduction}

In two-sided queues, customers and servers both arrive to the system and then wait to be matched. Many emerging applications and rapidly growing marketplaces can be modeled as such systems: e.g., Uber and Lyft where passengers are matched with drivers, Grubhub and DoorDash where customers are matched with meal delivery couriers, and crowdsouring platforms where tasks are matched to contributors. Most of these platforms use both dynamic pricing and dynamic matching as levers to facilitate market profitability and efficiency.

In this paper, we consider a canonical model of two-sided queues with multiple types of servers and multiple types of customers. Each type of customer is allowed to be matched with only a subset of server types. For example, in the case of ride-hailing marketplace, the types of servers (drivers) and customers are determined by their respective geographical locations - only customers and servers which are nearby will be compatible to be matched to each other - as well as other factors such as number of seats needed, vehicle type, and vehicle capacity. In the case of crowdsouring platforms, the types are determined by the nature of tasks and the expertise of contributors.

We assume that at each point in time, the system operator sets a price for each type of customer and server. Then, customers who are willing to pay and servers who are willing to serve for the 
quoted prices enter the system. They each wait in separate queues, until they are matched to a compatible counterpart. Once a pair is matched to each other, they will leave the system immediately in order to complete the service. The system operator earns a profit, which is equal to the difference between the price charged to the customer and the price quoted to the server.

We formulate the above system as an Markov decision process (MDP) in infinite horizon. The operator can vary the price for each type of customer and server, as well as decide when to match and which customer-server pair to match. The objective is to maximize the profits obtained by the system operator, while accounting for the queuing delay experienced by the customers and servers. We are especially interested in the behavior of systems with large traffic, where all of the arrival rates are scaled by a factor $\eta \rightarrow \infty$. Under this scaling regime, any policy that is within $o(\eta)$ of the optimal objective is asymptotically optimal.

The main challenge in this problem is the curse of dimensionality in solving the MDP. As the number of customer or server types increases, the dimension of the state space increases exponentially, even when the buffer size of the queue for each type is bounded by a constant. Thus, it is intractable to solve the exact MDP for large scale systems with multiple types, which are often encountered in practice. In this paper, we propose approximation technique to obtain near optimal solutions for the MDP.

The contributions of the paper are the following. We model a bipartite two-sided queuing network with stochastic arrivals as a Markov decision process (Section 2). In some special cases, we are able to show structural properties of the optimal dynamic pricing policy (Section 3). We then analyze a fluid model (Section 4). The revenue obtained by solving the fluid model is an upper bound on the achievable revenue under any policy. We propose a fluid pricing and max-weight matching policy, and show that the profit loss from the fluid revenue is $O(\sqrt{\eta})$; thus, it is asymptotically optimal. A noticeable feature of this policy is that it does not use the static optimal matching solution from the fluid model. Instead, this policy applies max-weight matching (motivated by Tassiulas and Ephremides (1992)) which is adaptive to queue lengths. We also propose a two-price and modified max-weight matching policy, and show that the profit loss from the fluid revenue is reduced to $O\left(\eta^{1 / 3}\right)$ (Section 5). Under a broad class of pricing policies, we further show that any matching policy will have a profit loss from the fluid revenue is lower bounded by $\Omega\left(\eta^{1 / 3}\right)$ (Section 6). Finally, we present an LP-based approximation technique and a constraint generation algorithm to solve the MDP efficiently (Section 7).

\subsection{Literature Review}

Dynamic Matching. Dynamic matching markets have widespread applications, for exam-

ple, in ride sharing (Banerjee et al. 2017), online marketplaces like Amazon.com or Ebay, kidney 
exchange (Roth et al. 2007, Anderson et al. 2017), payment processing networks (Sivaraman et al. 2018) among others. Now, we will discuss few previous work involving dynamic matching in the context of two sided queues.

Caldentey et al. (2009) and Adan and Weiss (2012) consider bipartite matching for two-sided queues on a first-come-first-served basis: each arriving customer is matched to a compatible server who has the earliest arrival time and has not been matched. Under this matching rule, they analyzed limiting results of matching rates between certain customer and server types. Furthermore, they deduced the necessary conditions on the frequency of arrivals for stability of the system and also derived the stationary distribution. Gurvich and Ward (2014) analyze a general multi-sided queuing system, where more than two sides may be present. The objective is to minimize finite time cost incurred due to queuing. They presented a matching algorithm and showed asymptotic optimality with rate of convergence $O(\sqrt{\eta})$ where arrivals are scaled by $\eta$.

$\mathrm{Hu}$ and Zhou (2018) studied a two-sided matching system similar to ours. The goal is to maximize the discounted reward obtained by matching customers and servers in a finite horizon, while accounting for the holding costs. They study conditions such that a priority rule is optimal. In addition, they present a matching algorithm based on fluid approximation and show that it is asymptotically optimal. The main distinction of $\mathrm{Hu}$ and Zhou (2018) with our paper is that they do not consider dynamic pricing. In addition, while they use fluid approximation to generate static matching decisions, we use max-weight algorithm to generate matching decisions that are adaptive to queue lengths (see discussion on max-weight algorithm below). Chen and Hu (2019) study dynamic pricing and matching for a single product/service with strategic customers and servers. Different than ours, their matching policy does not necessarily comply with first-come-first-served basis. Our model also allows different types of service.

Dynamic matching problems were also studied in the context of kidney exchanges albeit in a non-two-sided setting in Anderson et al. (2017), Akbarpour et al. (2019). Due to existing laws, pricing is usually not allowed in kidney exchanges. These papers study the value of "batching", i.e., holding compatible matching pairs in hope that better matching will arrive in future. However, both papers find that batching in general does not provide significant benefit.

Dynamic Pricing for Queues. Dynamic pricing is a common mechanism for increasing revenue. First we discuss the literature involving dynamic pricing in the context of single sided queue and later also present some papers involving two sided queues.

Low (1974a) is one of the earlier works studying dynamic pricing in a single sided queue. The paper considered price dependent customer arrivals with a finite buffer; the rewards include the payment by customers and holding costs incurred by the operator. Monotonicity of the optimal pricing 
policy is showed. It was later extended to infinite buffer capacity in Low (1974b). Chen and Frank (2001) considered a queuing model with customers who are sensitive to both waiting time and price.

They presented structural properties on optimal pricing decisions and monotonicity of optimal bias function. In the context of network services like call centers, Paschalidis and Tsitsiklis (2000) considered a system with finite total resource. They consider different types of price dependent customers arrivals which requests for a fraction of the resource. The objective is to find a pricing policy to maximize the revenue. They show multiple structural properties like concavity of value function and monotonicity of optimal policy.

Kim and Randhawa (2017) considers a single server queuing system and studies the benefit of dynamic pricing over static pricing. They consider that the customers are delay sensitive and consider similar objective as ours, which is to maximize the revenue. They present asymptotically optimal dynamic pricing policy and a two-price policy and also provide the rate of convergence of these policies. The main distinction of Kim and Randhawa (2017) with our paper is that they consider a single server queue, whereas we consider a network of two-sided queues where matching decisions are required.

A two-sided queueing model with both customer and server arrivals is studied by Nguyen and Stolyar (2018). They consider server arrival by invitation which is analogous to pricing for the servers. The customers and servers are allowed to abandon, which ensures stability. However, the focus in Nguven and Stolyar (2018) was to establish process level convergence, while the objective in our model is to maximize the profit of the system.

Several recent papers have studied dynamic pricing in the context of ride hailing systems (Korolko et al. 2018, Besbes et al. 2018, Hu et al. 2019). Baneriee et al. (2017, 2018) study a closed queuing network, where the number of cars in the system is a constant and the customers abandon the system if they are not matched immediately. Baneriee et al. (2017) provides a stateindependent pricing policy and prove the approximation ratio with respect to optimal pricing policy. (Banerjee et al. 2016) provides a state-dependent pricing policy and argue that the benefit of dynamic pricing is in the robustness of the performance of the system.

Dynamic pricing mechanisms have also been extensively studied in revenue management literature (Talluri and Van Ryzin 2006). In our model, we use dynamic pricing as a lever to increase system operator's profit while making the system stable. Note that dynamic pricing subsumes external control like admission control of customers and on-demand servers (rejection is equivalent to increasing prices to infinity).

Max-Weight Algorithm. In this work, we propose a max-weight matching algorithm for a two-sided queuing problem. This algorithm was first proposed by Tassiulas and Ephremides (1992) 
in the context of communication networks. After that, the max-weight algorithm and the backpressure algorithm, which is a generalization of the max-weight algorithm, are studied intensively in the literature. The book by Srikant and Ying (2014) provides an excellent summary. The performance of max-weight algorithm in the context of a switch operating in heavy traffic has been studied by Maguluri and Srikant (2016). The backpressure algorithm was used in the context of online ad matching in (Tan and Srikant 2012) and in the context of ride hailing in (Kanoria and Qian 2019).

\subsection{Notation}

Throughout the paper, vectors are boldfaced. We use $\langle.,$.$\rangle to denote dot product between two$ vectors. All the functions applied on a vector is component wise, e.g. $F(\boldsymbol{\lambda})$ is defined to be $\left(F\left(\lambda_{1}\right), \ldots, F\left(\lambda_{m}\right)\right)$. Moreover, $\mathbf{a} \leq \mathbf{b}$ means that all the components of $\mathbf{a}$ is less than or equal to corresponding components of $\mathbf{b}$. We use $i$ and superscript (1) to denote a server type and $j$ and superscript (2) to denote a customer type. In addition, we define the vector $\mathbf{e}_{j}^{(2)}$ and $\mathbf{e}_{i}^{(1)}$ as a vector with all zeros except a one for type $j$ customer and type $i$ server respectively.

We define $\mathbb{E}[$.$] for expected value of a random variable and the conditional expectation \mathbb{E}[. \mid x]$ is written as $\mathbb{E}_{x}[$.$] for the ease of notation. We denote the set \{1,2, \ldots, n\}$ by $[n]$. We denote the vector with all 1's as $\mathbf{1}_{n}$ with size $n$; we will sometimes omit the subscript if the size of the vector is clear from the context wherever it is used. In addition, we define $\mathbf{0}_{n}$ as the vector with all 0's of size $n$ and we omit the subscript if the size of the vector is clear from the context. For the vectors $\mathbf{x} \in \mathbb{R}^{n}$ and $\mathbf{y} \in \mathbb{R}^{m}$, we denote the concatenated vector of $n+m$ dimension by $(\mathbf{x}, \mathbf{y})$. If $\mathbf{x}$ and $\mathbf{y}$ are of the same dimension, we denote the component wise product by $\mathbf{x} \circ \mathbf{y}$.

\section{Model}

We model the multiple types of customers and servers by a bipartite graph $G\left(N_{1} \cup N_{2}, E\right)$, where $N_{1}$ is the set of server types with $\left|N_{1}\right|=n$ and $N_{2}$ is the set of customers type with $\left|N_{2}\right|=m$. Here, $E$ is the set of edges in the bipartite graph between customer and server types which are compatible with each other. In other words, $(i, j) \in E$ if type $j$ customer can be served by type $i$ server, as illustrated in Figure 1, Each node in the bipartite graph is a queue of customers or servers waiting to be matched with any one of the compatible counterpart. Our convention is to refer the incoming customers as demand and incoming servers as supply.

At each point in time, the system operator posts a price for each type of customers and servers. Customers willing to pay the quoted price, as well as servers who are willing to provide their service for the quoted price, are admitted to the system. Thus, the system operator can vary the prices of the customers and servers to vary their arrival rate. Once they are in the system, they wait in queues until they are picked and matched. First-come-first-serve (FCFS) discipline is employed 
Figure 1 A bipartite graph for two-sided queues.

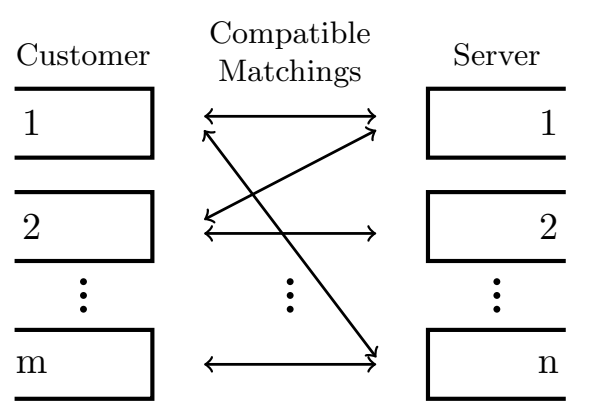

for each queue separately. Note that, FCFS may not hold among different types of customers and servers (unlike Adan and Weiss 2012, Caldentev et al. 2009); it will rather depend on the matching policy used. Once the customer is matched with a compatible server, we assume that they depart from the system instantaneously to complete the transaction. Our objective is to find a joint pricing and matching policy under which the system is stable (positive recurrent) and the long-run average profit is maximized.

Next, we present some notation and assumptions on the pricing decisions and the matching decisions. We assume that customers and servers arrive according to Poisson processes with rate $\boldsymbol{\lambda} \in \mathbb{R}_{+}^{m}$ and $\boldsymbol{\mu} \in \mathbb{R}_{+}^{n}$ respectively. For each customer type $j \in[m]$, there exists a demand curve $F_{j}: \lambda_{j} \rightarrow p_{j}^{(2)}$, such that if the system operator sets a price $p_{j}^{(2)}$, the resulting arrival rate is $\lambda_{j}$. Similarly, for each server type $i \in[n]$, the system operator can set a price denoted by $p_{i}^{(1)}$, which results in a server arrival rate $\mu_{i}$ determined by the respective supply curve $G_{i}: \mu_{i} \rightarrow p_{i}^{(1)}$. We make the following assumption on the supply and demand curve.

Assumption 1. The supply curves, $G_{i}, \forall i \in[n]$ are strictly increasing and twice continuously differentiable. The demand curves, $F_{j}, \forall j \in[m]$ are strictly decreasing and twice continuously differentiable.

The above assumption states that offering higher price to the servers will result in higher server arrival rate, offering the service for lower prices will result in higher customer arrival rate, and all curves are smooth.

In addition, we define the revenue and cost functions as $r_{j}^{(2)}\left(\lambda_{j}\right) \triangleq \lambda_{j} F_{j}\left(\lambda_{j}\right)$ for all $j \in[m]$ and $r_{i}^{(1)}\left(\mu_{i}\right) \triangleq \mu_{i} G_{i}\left(\mu_{i}\right)$ for all $i \in[n]$. We make the following assumption on the revenue and cost functions.

Assumption 2. The revenue function $r_{j}^{(2)}\left(\lambda_{j}\right)$ is concave $\forall j \in[m]$. The cost function $r_{i}^{(1)}\left(\mu_{i}\right)$ is convex $\forall i \in[n]$.

The assumption on revenue function follows from the law of diminishing marginal return: as we increase the customer arrival rate, the marginal revenue $d r_{j}^{(2)}\left(\lambda_{j}\right) / d \lambda_{j}$ decreases, which implies 
that the revenue function $r_{j}^{(2)}\left(\lambda_{j}\right)$ is concave. Similar assumption is often assumed in the revenue management and queueing literature (see e.g. (Kim and Randhawa 2017)). As for the servers, we assume the marginal cost $d r_{i}^{(1)}\left(\mu_{i}\right) / d \mu_{i}$ increases with $\mu_{i}$, since it becomes harder to recruit servers when we try to increase server arrival rate. This implies that the cost function $r_{i}^{(1)}$ is convex.

Besides setting prices, the system operator also uses matching to govern the process of the queueing system. At any given time, suppose $q_{j}^{(2)}$ is the number of type $j$ customers waiting in the queue and $q_{i}^{(1)}$ is the number of type $i$ servers. We denote by $x_{i j}$ the number of type $i$ servers to be matched to type $j$ customers. The number of matchings should be bounded by both the number servers and the number of customers currently waiting in the queue, so we have

$$
\begin{aligned}
x_{j}^{(2)} & =\sum_{i=1}^{n} x_{i j} \leq q_{j}^{(2)}, \forall j \in[m] \\
x_{i}^{(1)} & =\sum_{j=1}^{m} x_{i j} \leq q_{i}^{(1)}, \forall i \in[n] \\
x_{i j} & =0, \forall(i, j) \notin E .
\end{aligned}
$$

The set of matches that satisfies (1a) -(1c) is denoted by $X(\mathbf{q})$. When a pair of customer and server is matched by the system, they both depart from the system. Since a customer is only compatible to a subset of server types, the system operator may have an incentive for batching, i.e., holding some customers or servers in queue in order to achieve better matches in future.

Example: Ride Hailing. An application of the two-sided queueing model is the ride hailing system. In this system, the customer and server (drivers) types, as well as the matching compatibility graph, are determined by their geographical location. A simple example with three regions is shown in Figure 2, (Here, we ignore issues such as vehicle capacity and number of seats requested by customers, which may lead to additional customer and server types.) Based on the price quoted to customers, only a fraction of them who open the app will book a ride, which determines the customer arrival rate. Similarly, based on the price quoted to the drivers, they will choose whether or not to provide service. Thus, the arrival rate of customer and drivers are price dependent and is governed by the demand and supply curve of each region. Once a customer confirms the price and books a ride, the system operator can determine which driver (from what region) should be matched to the customer. If the driver accepts the ride request, then it immediately becomes unavailable for any other ride requests (departing from the system). After the ride is complete, the car becomes available again, possibly in a different region. A simplifying assumption in our model is that we treat a driver who completes the service and re-enters the system the same as a new arrival. 
Figure 2 A ride hailing system with three regions.

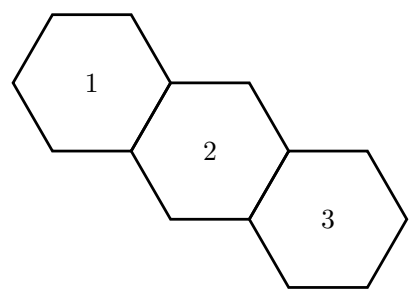

(a) Geographical locations

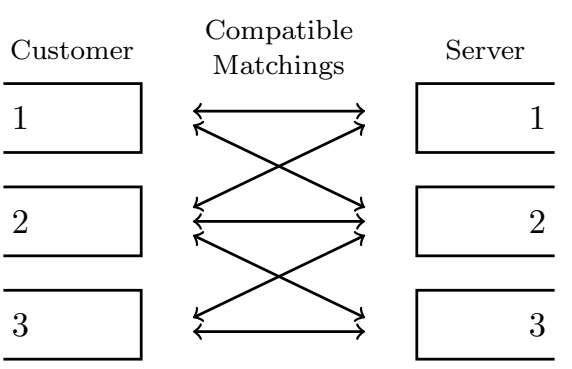

(b) Bipartite graph

We assume that riders can only be matched to cars in their own region or any neighboring regions. The two sided system generated from the map is shown in subfigure (b).

\subsection{CTMDP Formulation}

We now formally define the system operator's decision process as a continuous time Markov decision process (CTMDP), including its states, actions, rewards, and objective.

With every customer arrival, the system operator earns a reward equal to the price set for that customer type; similarly, the system operator pays a cost with each server arrival equal to the price set for that server type. Additionally, the system operator incurs a holding cost proportional to the number of customers and servers waiting in the system per unit time. The state is represented by the queue lengths of all customer and server types, $\mathbf{q} \in \mathbb{Z}_{+}^{n+m}$. We denote the state space by $S$. By Assumption 1, prices and arrival rates have a one-one correspondence. Thus, rather than using price as the action, we equivalently use arrival rates $\boldsymbol{\lambda}(\mathbf{q}), \boldsymbol{\mu}(\mathbf{q})$ for all $\mathbf{q} \in S$ as the action. The arrival rates much satisfy $\lambda_{j} \in \operatorname{dom}\left(F_{j}\right)$ for all $j \in[m]$ and $\mu_{i} \in \operatorname{dom}\left(G_{i}\right)$ for all $i \in[n]$. Here, $\operatorname{dom}(F)$ is the domain of the function $F$. In addition, the matching decisions $\mathbf{x}(\mathbf{q}) \in X(\mathbf{q})$ are also in the action space (see Eq (1a $)-(1 \mathrm{c})$ ). Thus, action is a tuple $(\boldsymbol{\lambda}, \boldsymbol{\mu}, \mathbf{x}) \in \mathbb{R}^{2(m+n)}$. The objective is to find a pricing and matching policy such that the long run average profit earned by the system operator is maximized. We are only interested in the pricing policies under which make the system stable in the long run. The following definition of stability will be used.

Definition 1. A joint pricing and matching policy is said to be stable, if the continuous-time Markov chain (CTMC) induced from this policy has a positive recurrent communicating class that contains the state $\mathbf{q}=\mathbf{0}$.

The MDP advances as follows: Upon each customer or server arrival, we match customers and servers by setting $\mathbf{x} \in X(q)$. We allow $\mathbf{x}=\mathbf{0}_{m+n}$, i.e., none of the customer-server pairs are matched, even when there exists feasible matching. Immediately after the arrival, we may change the prices for any customer and server type. Based on the prices set, future type $j$ customers arrive following $\operatorname{Poisson}\left(\lambda_{j}\right)$ for all $j \in[m]$, and type $i$ servers arrive following $\operatorname{Poisson}\left(\mu_{i}\right)$ for all $i \in[n]$. 
REMARK 1 (Discussion Of WAITING COSTS). Our model assumes all customers and servers are patient, and the system operator pays a penalty cost proportional to their waiting time. As an alternative approach, Kim and Randhawa (2017) considered a queuing model without such waiting cost penalty - instead, they assume customers are strategic and will not joint the queue if their valuation of the service is less than their expected waiting cost. Compared to our model, the assumption by Kim and Randhawa (2017) may be more appropriate for some applications of twosided queues such as ride hailing. Nevertheless, Kim and Randhawa (2017) point out that their assumption of strategic customers is essentially equivalent to a model with patient customers by explicitly accounting for waiting cost, i.e., our approach. We refer readers to Kim and Randhawa (2017), Section 6.1 for more details.

\subsection{Uniformization}

Instead of working with the CTMDP, we use the well-known uniformization technique (Puterman 1994) to obtain an equivalent discrete-time Markov Decision Process chain (DTMDP), which will simplify our analysis. The uniformized process is as follows. We first choose a uniformization parameter $c$ defined below.

Definition 2. Suppose there exists $\boldsymbol{\lambda}_{\max }$ and $\boldsymbol{\mu}_{\max }$ such that for any price vector $\mathbf{p}$ we have,

$$
\begin{aligned}
& \boldsymbol{\lambda}(\mathbf{p}) \leq \boldsymbol{\lambda}_{\max } \\
& \boldsymbol{\mu}(\mathbf{p}) \leq \boldsymbol{\mu}_{\max }
\end{aligned}
$$

Let $c$ be any constant such that

$$
c \geq\left\langle\mathbf{1}_{m}, \boldsymbol{\lambda}_{\max }\right\rangle+\left\langle\mathbf{1}_{n}, \boldsymbol{\mu}_{\max }\right\rangle .
$$

The uniformized DTMDP is endowed with the same states and actions as the CTMDP defined in Section 2.1. All the rewards and costs will be scaled by $1 / c$. In each period, there is at most one customer or server arrival. Let $\mathbf{p}$ be price vector. The probability that a type $j$ customer arrives in one period is $\lambda_{j}(\mathbf{p}) / c$. The probability that a type $i$ server arrives in one period is $\mu_{i}(\mathbf{p}) / c$. Otherwise, no arrival happens in this period. The decision maker is allowed to make any number of matchings as long as they satisfy constraints (1a $)-(1 \mathrm{c})$.

Bellman equation. To find the optimal pricing and matching policy, we start by writing the Bellman equation for the uniformized DTMDP. Let $Z(\mathbf{q})=\left[0, \boldsymbol{\lambda}_{\max }\right] \cup\left[0, \boldsymbol{\mu}_{\max }\right] \cup \mathbf{x}(\mathbf{q})$ be the set of feasible prices and matchings for queue length $\mathbf{q}$. We use $\mathbf{z}$ to denote a joint action vector, which includes pricing decisions $\boldsymbol{\lambda}, \boldsymbol{\mu}$ and matching decisions $\mathbf{x} \in X(\mathbf{q})$. 
The Bellman equation can be written as

$$
h(\mathbf{q})+\frac{\gamma}{c}=\max _{\mathbf{z} \in Z(\mathbf{q})}\left\{\frac{\mathcal{R}(\mathbf{q}, \mathbf{z})}{c}+\mathbb{E}_{\mathbf{q}, \mathbf{z}}[V(h, \mathbf{q}, \mathbf{z})]\right\}, \quad \forall \mathbf{q} \in S
$$

where

$$
\begin{aligned}
\mathcal{R}(\mathbf{q}, \mathbf{z})= & \langle F(\boldsymbol{\lambda}), \boldsymbol{\lambda}\rangle-\langle G(\boldsymbol{\mu}), \boldsymbol{\mu}\rangle-s\langle\mathbf{1}, \mathbf{q}\rangle, \\
\mathbb{E}_{\mathbf{q}, \mathbf{z}}[V(h, \mathbf{q}, \mathbf{z})]= & \sum_{j=1}^{m} \frac{\lambda_{j}}{c} h\left(\mathbf{q}+\mathbf{e}_{j}^{(2)}-\mathbf{x}\right)+\sum_{i=1}^{n} \frac{\mu_{i}}{c} h\left(\mathbf{q}+\mathbf{e}_{i}^{(1)}-\mathbf{x}\right) \\
& +\left(1-\sum_{j=1}^{m} \frac{\lambda_{j}}{c}-\sum_{i=1}^{n} \frac{\mu_{i}}{c}\right) h(\mathbf{q}),
\end{aligned}
$$

The solution $\gamma$ to the above equations is the optimal average profit in the infinite horizon. The value $h(\mathbf{q})$ is the bias function, which can be interpreted as a bias function associated with state $\mathbf{q}$ (Bertsekas 2007). Note that the Bellman equation holds because our assumption of stable policies (see Definition 1).

In the Bellman equation, $\mathcal{R}(\mathbf{q}, \mathbf{z})$ is the expected revenue for the state $\mathbf{q}$ and action $\mathbf{z}$. The revenue term is the net of what the customer pays and server gets along with a penalty proportional to the sum of the queue lengths. The term $\mathbb{E}_{\mathbf{q}, \mathbf{z}}[V(h, \mathbf{q}, \mathbf{z})]$ is the expectation of the bias function function $h$ after one transition in the uniformized process where $\mathbf{q}$ is the queue length, $\mathbf{z}$ is the chosen action at the current decision epoch. The expectation is taken with respect to arrival probabilities of the uniformized DTMDP.

\section{Monotonicity of the Optimal Prices (Single Link Two-Sided Queue)}

In this section, we consider the special case of our model with $n=1$ and $m=1$, i.e., a single link two-sided queue given in Fig. 3. The goal of this section is to establish structural properties of the optimal pricing policy by analyzing this simple system, which will motivate our pricing policies for more complex systems.

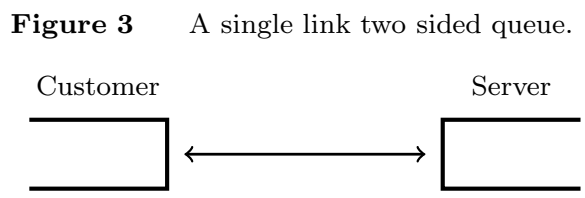

In a single link system, there is no incentive for the system operator to hold customers or servers: Whenever possible, we should match the incoming arrival immediately. Thus, at any point of time, there can only be either customers or servers waiting in the system. This enables up to reduce the state space by letting $q=q^{(2)}-q^{(1)}$, the difference between the number of customers and servers 
waiting in the system. Note that, $q$ can be either positive or negative. Using $q$ as the system state, the Bellman equation (3) becomes

$$
h(q)=\max _{\mu \geq 0, \lambda \geq 0}\left[\frac{F(\lambda) \lambda-G(\mu) \mu}{c}-\frac{s|q|}{c}-\frac{\gamma}{c}+\frac{\lambda}{c} h(q+1)+\left(1-\frac{\mu+\lambda}{c}\right) h(q)+\frac{\mu}{c} h(q-1)\right], \forall q \in S
$$

where $c$ is a uniformization parameter (see Definition 21). In the equation above, as we have $n=$ $m=1$, we omit the subscripts for different type of customers and servers for the ease of notation. We now present the monotonicity result below.

Proposition 1. For a single link two-sided queue, there exists an optimal pricing policy $\mathbf{p}(q)=$ $\left(p^{(1)}(q), p^{(2)}(q)\right)$, where both the server price $p^{(1)}(q)$ and the customer price $p^{(2)}(q)$ increases monotonically with the system state $q$.

To prove this result, we first show that the difference of bias functions $h(q)-h(q-1)$ is monotonically decreasing in $q$ by an inductive argument on the iterates of the relative value iteration algorithm. Then we use the optimality conditions on the Bellman equation to show that the optimal pricing policy is monotonic. The complete proof can be found in Appendix B, This result motivates us to search for the optimal pricing policy in the restricted space of monotonic pricing policies, which will be presented in Section 7 .

\section{Asymptotic Optimality of Fluid Solution}

In this section, we consider fluid approximation of the queueing system where random arrivals are replaced by their expectations. Based on the fluid model, we propose a static pricing and max-weight matching policy and show that it is asymptotically optimal.

\subsection{Fluid Model}

We consider a deterministic optimization problem to maximize the average long run revenue defined as follows. Let $\tilde{\boldsymbol{\lambda}}=\mathbb{E}[\boldsymbol{\lambda}(\mathbf{q})]$ and $\tilde{\boldsymbol{\mu}}=\mathbb{E}[\boldsymbol{\mu}(\mathbf{q})]$ be the average arrival rate of the customers and servers, respectively, where the expectation is taken with respect to the stationary distribution under any policy. Similarly, let $\chi_{i j}=\mathbb{E}\left[x_{i j}(\mathbf{q})\right]$ be the average rate of type $i$ server matched to the type $j$ customer for all $(i, j) \in E$. The fluid model is defined as

$$
\begin{array}{ll}
\pi^{*}=\max _{(\tilde{\boldsymbol{\lambda}}, \tilde{\boldsymbol{\mu}}, \boldsymbol{\chi})} & \langle F(\tilde{\boldsymbol{\lambda}}), \tilde{\boldsymbol{\lambda}}\rangle-\langle G(\tilde{\boldsymbol{\mu}}), \tilde{\boldsymbol{\mu}}\rangle \\
\text { subject to } & \tilde{\lambda}_{j}=\sum_{i=1}^{n} \chi_{i j}, \forall j \in[m], \\
& \tilde{\mu}_{i}=\sum_{j=1}^{m} \chi_{i j}, \forall i \in[n], \\
& \chi_{i j}=0, \forall(i, j) \notin E, \chi_{i j} \geq 0, \forall(i, j) \in E .
\end{array}
$$


Here, Eqs (7b) and (7c) are the balance equations for the number of customers and servers matched. Eq (7d) specifies that matching is only allowed among compatible customer-server pairs. Intuitively, it is easy to see that these constraints are necessary, because if Eqs (7b)-(7dd) do not hold, then some customer or server types will keep accumulating over time. Thus, if we solve the optimization program (17) to maximize the revenue, we will get an upper bound on the achievable revenue under any pricing and matching policy which makes the system stable. This is formally shown in the following proposition.

Proposition 2. The optimal value of the fluid problem (7) is an upper bound on the long run expected profit rate under any pricing and matching policy that makes the system stable.

Sketch Proof. We first show that under any pricing and matching policy, the constraints in the fluid problem are necessary but not sufficient conditions. Intuitively, the average arrival rates of customers and servers should be able to balance each other out. Next, we show that the average revenue obtained under any pricing and matching policy is less than equal to the fluid objective function. These two conditions together implies that fluid problem will give us an upper bound on the revenue obtained under any pricing and matching policy. The complete proof can be found in Appendix C.

\subsection{Fluid Pricing Policy}

In this section, we study the system in an asymptotic regime where the arrival rates of all customer and server types are scaled by a factor of $\eta$.

Definition 3 (Asymptotic Regime). Consider a family of two-sided queueing systems associated with the same bipartitle graph $G\left(N_{1} \cup N_{2}, E\right)$ parametrized by $\eta \in \mathbb{N}$. For the $\eta^{\text {th }}$ system, the demand and supply curves satisfy $F^{\eta}(\eta \boldsymbol{\lambda})=F(\boldsymbol{\lambda})$ for all $\boldsymbol{\lambda} \in \mathbb{R}_{+}^{m}$ and $G^{\eta}(\eta \boldsymbol{\mu})=G(\boldsymbol{\mu})$ for all $\boldsymbol{\mu} \in \mathbb{R}_{+}^{n}$. The asymptotic regime is defined as $\eta \rightarrow \infty$.

Definition 4 (Profit Loss). The profit loss of a policy, denoted by $L^{\eta}$, is the difference between the optimal value of the fluid model, denoted by $\pi_{*}^{\eta}$, and the long run average profit (including the penalty incurred due to waiting) under that policy.

According to Definition [3, it is easily verified that the fluid solution to the $\eta^{\text {th }}$ system is given by $\eta \boldsymbol{\lambda}^{*}$ and $\eta \boldsymbol{\mu}^{*}$, where $\boldsymbol{\lambda}^{*}$ and $\boldsymbol{\mu}^{*}$ is the optimal solution of the fluid model (7). The optimal value of the $\eta^{\text {th }}$ fluid model is $\pi_{*}^{\eta}=\eta \pi_{*}$. Therefore, if the profit loss of a policy is sublinear in $\eta$, namely $L^{\eta}=o(\eta)$, we say the policy is asymptotically optimal in our asymptotic regime. 
Now, we use the fluid model to define a fluid pricing policy, which we will prove to be asymptotically optimal. Recall that the optimal solution of (7) by $\boldsymbol{\lambda}^{*}, \boldsymbol{\mu}^{*}$. We consider the following pricing policy:

$$
\begin{aligned}
& \lambda_{j}(\mathbf{q})=\left\{\begin{array}{l}
\lambda_{j}^{*}, \text { if } q_{j}^{(2)}<q_{\max }^{\eta}, \\
0 \text { otherwise, }
\end{array}\right. \\
& \mu_{i}(\mathbf{q})=\left\{\begin{array}{l}
\mu_{i}^{*}, \text { if } q_{i}^{(1)}<q_{\max }^{\eta}, \\
0 \text { otherwise, }
\end{array} \quad \forall i \in[n] .\right.
\end{aligned}
$$

Here, $q_{\max }^{\eta}$ denotes the maximum queue length; it is a parameter that depends on $\eta$, which will be specified later.

We now define the max-weight matching policy (Algorithm 1). Whenever there is either a customer or a server arrival, if any of the compatible counterparts of the arrival is waiting in the queue, then we match it to the type with most number of customers/servers waiting in that queue. On the other hand, if all the compatible counterparts' queues are empty, then that arrival will then wait in the queue. More specifically, let $k$ be the index of arrivals to the system, which is our decision epoch. We denote the arrival vector by $\mathbf{a}(k) \in\{0,1\}^{m+n}$. That is, $\mathbf{a}(k)$ has one component equal to one, which corresponds to the arrival type, and all the other components are zero. We define $y_{i j}^{(1)}(k) \in\{0,1\}$ to be the decision of matching a type $j$ customer to a type $i$ server at the arrival of a type $i$ server. Similarly, we also define $y_{i j}^{(2)}(k) \in\{0,1\}$ to be decision of matching a type $i$ server to type $j$ customer at the arrival of type $j$ customer.

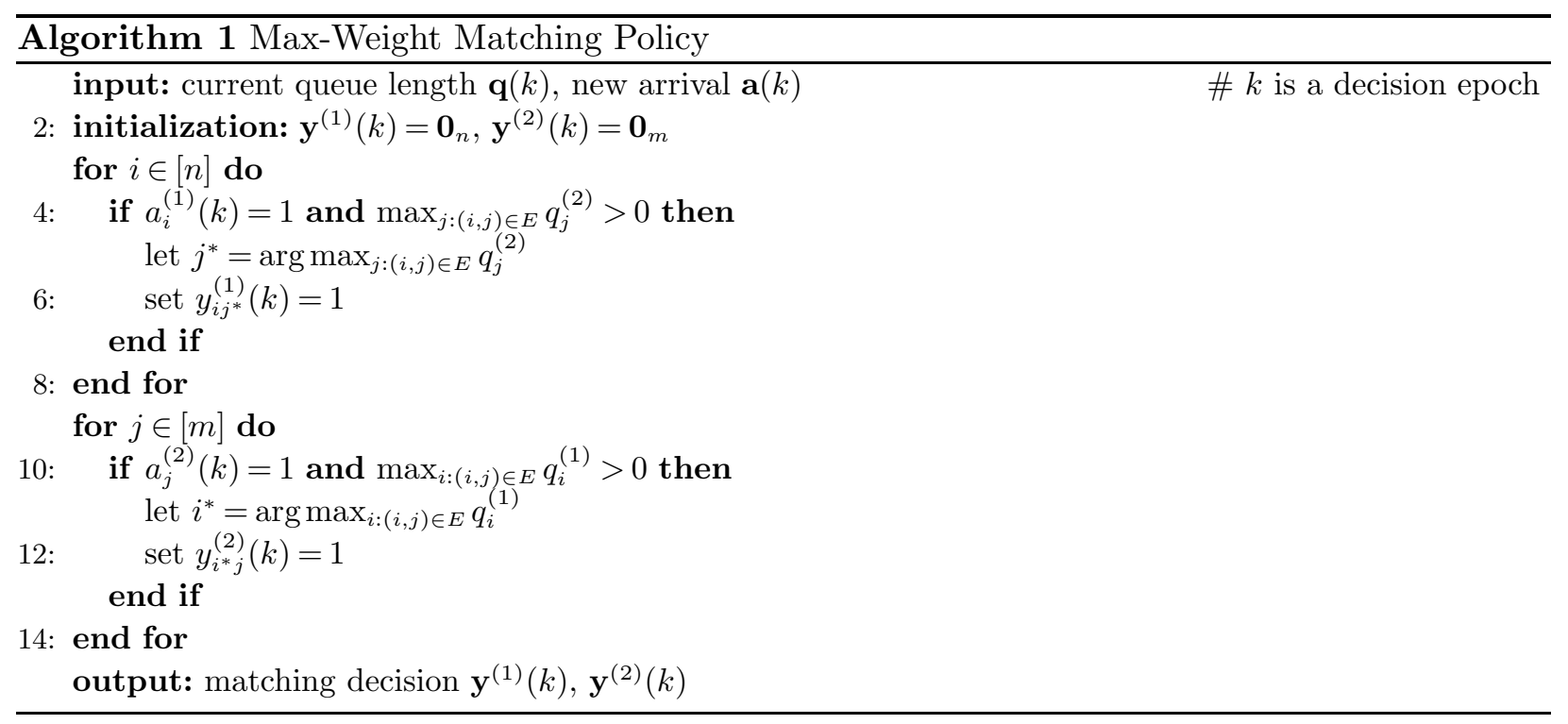

The main intuition of the fluid pricing policy is the following. We note that the profit rate of the fluid pricing policy is equal to $\eta \pi^{*}$ when all queues are below their maximum buffer capacity $\mathbf{q}^{\eta}$. 
If the capacity of any customer queue is full, namely $q_{j}^{(2)}=q_{\max }^{\eta}$, then all future arrivals to queue $j$ will be rejected until at least one customer waiting in queue $j$ is matched. Thus, we will lose a fraction of revenue due to rejections. More specially, let $\pi^{\eta}$ be the expected profit of the fluid pricing policy. Let $\mathbf{I}^{(1)}\left(q_{\max }^{\eta}\right)$ be a (vector) indicator function representing whether server queues are at the maximum capacity, and let $\mathbf{I}^{(2)}\left(q_{\max }^{\eta}\right)$ be a (vector) indicator function representing whether customer queues are at the maximum capacity. Then, it holds that

$$
\begin{aligned}
L^{\eta}= & \pi_{*}^{\eta}-\left(\pi^{\eta}-s\left\langle\mathbf{1}_{m+n}, \mathbb{E}[\mathbf{q}]\right\rangle\right) \\
= & \eta\left(\left\langle F\left(\boldsymbol{\lambda}^{*}\right), \boldsymbol{\lambda}^{*}\right\rangle-\left\langle G\left(\boldsymbol{\mu}^{*}\right), \boldsymbol{\mu}^{*}\right\rangle\right)-\left\langle F\left(\boldsymbol{\lambda}^{*}\right), \eta \boldsymbol{\lambda}^{*} \circ\left(\mathbf{1}-\mathbb{E}\left[\mathbf{I}^{(2)}\left(q_{\max }^{\eta}\right)\right]\right)\right\rangle \\
& \quad-\eta\left\langle G\left(\boldsymbol{\mu}^{*}\right), \boldsymbol{\mu}^{*} \circ\left(\mathbf{1}-\mathbb{E}\left[\mathbf{I}^{(1)}\left(q_{\max }^{\eta}\right)\right]\right)\right\rangle+s\left\langle\mathbf{1}_{m+n}, \mathbb{E}[\mathbf{q}]\right\rangle \\
= & \eta\left(\left\langle F\left(\boldsymbol{\lambda}^{*}\right),\left(\boldsymbol{\lambda}^{*} \circ \mathbb{E}\left[\mathbf{I}^{(2)}\left(q_{\max }^{\eta}\right)\right]\right)\right\rangle-\left\langle G\left(\boldsymbol{\mu}^{*}\right),\left(\boldsymbol{\mu}^{*} \circ \mathbb{E}\left[\mathbf{I}^{(1)}\left(q_{\max }^{\eta}\right)\right]\right\rangle\right)+s\left\langle\mathbf{1}_{m+n}, \mathbb{E}[\mathbf{q}]\right\rangle,\right.
\end{aligned}
$$

where the first equality follows from Definition 4, and the second equality uses the definition of the fluid pricing policy. As a result, Eq (9) shows that the profit loss of the fluid pricing policy depends on critically the design parameter $q_{\max }^{\eta}$. If we increase the buffer capacity $q_{\max }^{\eta}$, then the probability of dropping customers/servers will reduce, i.e. $\mathbb{E}\left[\mathbf{I}\left(\mathbf{q}_{\max }^{\eta}\right)\right]$ will decrease. However, increasing the buffer capacity will lead to increasing in the expected queue lengths, which will increase the penalty incurred due to waiting. Thus, we choose buffer capacity to balance the tradeoff in order to minimize the overall profit loss. Precisely, we will see that choosing $q_{\max }^{\eta} \sim \sqrt{\eta}$ will result in $\mathbb{E}\left[\mathbf{I}\left(\mathbf{q}_{\max }^{\eta}\right)\right] \sim \eta^{-1 / 2}$ and $\mathbb{E}\left[\left\langle\mathbf{1}_{m+n}, \mathbf{q}\right\rangle\right] \sim \sqrt{\eta}$, which attains the optimal profit loss.

Theorem 1. Suppose a family of two-sided queues is given by the bipartite graph $G\left(N_{1} \bigcup N_{2}, E\right)$ parameterized by $\eta$. The profit loss $L^{\eta}$ under the fluid pricing (Eq (8) ) and max-weight matching (Algorithm 1) is $O(\sqrt{\eta})$, where $q_{\max }^{\eta}=\gamma \sqrt{\eta}$ for any positive constant $\gamma$.

Sketch Proof. We apply Eq (9) to bound of the profit loss $L^{\eta}$. By our choice of $q_{\max }^{\eta}$ and the definition of the fluid pricing policy defined in (8), it is easily verified that the expected queue length $\mathbb{E}\left[\left\langle\mathbf{1}_{m+n}, \mathbf{q}\right\rangle\right]=O(\sqrt{\eta})$. In order to bound the probability of dropping a customer or a server, we consider a quadratic Lyapunov function on the queue length vector q. Using the moment bound theorem (Hajek 2006), we can show that the probability of rejection is $O(1 / \sqrt{\eta})$. The proof details are deferred to Appendix D.

In addition, it can be shown that the $O(\sqrt{\eta})$ profit loss rate cannot be improved using the fluid pricing policy, even for a single link two-sided queue (see Figure 3). More specifically, we have the following result.

Proposition 3. For a family of single link two-sided queue parametrized by $\eta$, any fluid pricing policy will have a profit loss $L^{\eta}$ that is at least $\Omega(\sqrt{\eta})$. The choice of $q_{\max }^{\eta}=\gamma \sqrt{\eta}$ for any positive constant $\gamma$ provides the optimal profit loss rate $\Theta(\sqrt{\eta})$.

The proof of the proposition is presented in Appendix E. 


\section{Asymptotic Optimality of Two-Price Policy}

A main drawback of the fluid pricing policy is that the prices are fixed at those given by the fluid solution. This motivates us to consider a generalization of the fluid pricing policy, which leads to a "two-price" policy. Here, we introduce additional parameters $\boldsymbol{\theta} \in \mathbb{R}_{+}^{m}, \boldsymbol{\phi} \in \mathbb{R}_{+}^{n}$ and $\sigma^{\eta}$, which governs the arrival rates of the customers and servers respectively when the queue length is greater than a certain threshold $\tau_{\max }^{\eta}$. The two-price policy is defined as follows:

$$
\begin{aligned}
\lambda_{j}^{\eta} & =\left\{\begin{array}{ll}
\eta \lambda_{j}^{*} & \text { if } q_{j}^{(2)} \leq \tau_{\max }^{\eta}, \\
\eta \lambda_{j}^{*}-\theta_{j} \sigma^{\eta} & \text { otherwise }
\end{array} \forall j \in[m],\right. \\
\mu_{i}^{\eta} & =\left\{\begin{array}{ll}
\eta \mu_{i}^{*} & \text { if } q_{i}^{(1)} \leq \tau_{\max }^{\eta}, \\
\eta \mu_{i}^{*}-\phi_{i} \sigma^{\eta} & \text { otherwise }
\end{array} \forall i \in[n],\right.
\end{aligned}
$$

where we assume $\sigma^{\eta} / \eta \rightarrow 0$ as $\eta \rightarrow \infty$ as it is favourable to get closer to the fluid solution. In this policy, we set a threshold $\tau_{\max }^{\eta}$ for all customer and server types; we use the fluid arrival rates until this threshold, and then reduce the arrival rates by $\theta_{j} \sigma^{\eta}$ outside this threshold for type $j$ customer. Similarly, we reduce the server arrival rates outside a threshold by $\phi_{i} \sigma^{\eta}$ for type $i$ server. Here, $\tau_{\max }^{\eta}, \sigma^{\eta}, \boldsymbol{\theta}$ and $\phi$ are parameters that will be specified later. Our convention is to use superscript $\eta$ to denote any parameter or quantity that is associated with the $\eta^{\text {th }}$ system. Intuitively, for any type of customer/server, if we increase $\sigma^{\eta}$, the queue length will have a larger negative drift when it exceeds the threshold $\tau_{\max }^{\eta}$, so the expected queue length $\mathbb{E}\left[\left\langle\mathbf{1}_{m+n}, \mathbf{q}\right\rangle\right]$ will be smaller. However, if $\sigma^{\eta}$ are too large, the arrival rates outside the threshold $\tau_{\max }^{\eta}$ will be far from the optimal fluid arrival rates, which will result in a larger profit loss. Thus, there is a trade-off between the expected queue length and profit loss. For the matching algorithm associated with the two-price policy, we propose a modification on the max-weight policy. Let $\left(\boldsymbol{\lambda}^{*}, \boldsymbol{\mu}^{*}, \boldsymbol{\chi}^{*}\right)$ be the optimal solution to the fluid problem (7). Let $E^{*}=\left\{(i, j) \in E: \chi_{i j}^{*}>0\right\}$ be the support of $\chi^{*}$. We consider a max-weight algorithm analogous to Algorithm 1, except that we restrict matchings to the subset of edges $E^{*}$. This matching algorithm is defined in Algorithm 2, which will be referred to as the modified max-weight policy.

The following theorem provides a bound on the asymptotic performance of the two-price policy as $\eta$ tends to infinity.

TheOREm 2. Suppose a family of two-sided queues is given by the bipartite graph $G\left(N_{1} \bigcup N_{2}, E\right)$ parametrized by $\eta$. The profit loss $L^{\eta}$ under the two-price policy (Eq (10),(11)) and the modified

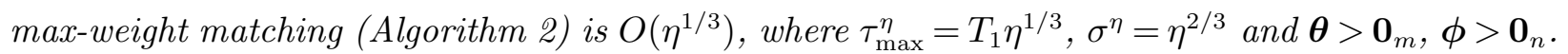

The profit loss for the two-price policy is $O\left(\eta^{1 / 3}\right)$, which is better than the $O(\sqrt{\eta})$ loss in the fluid pricing policy in Section 4 . 


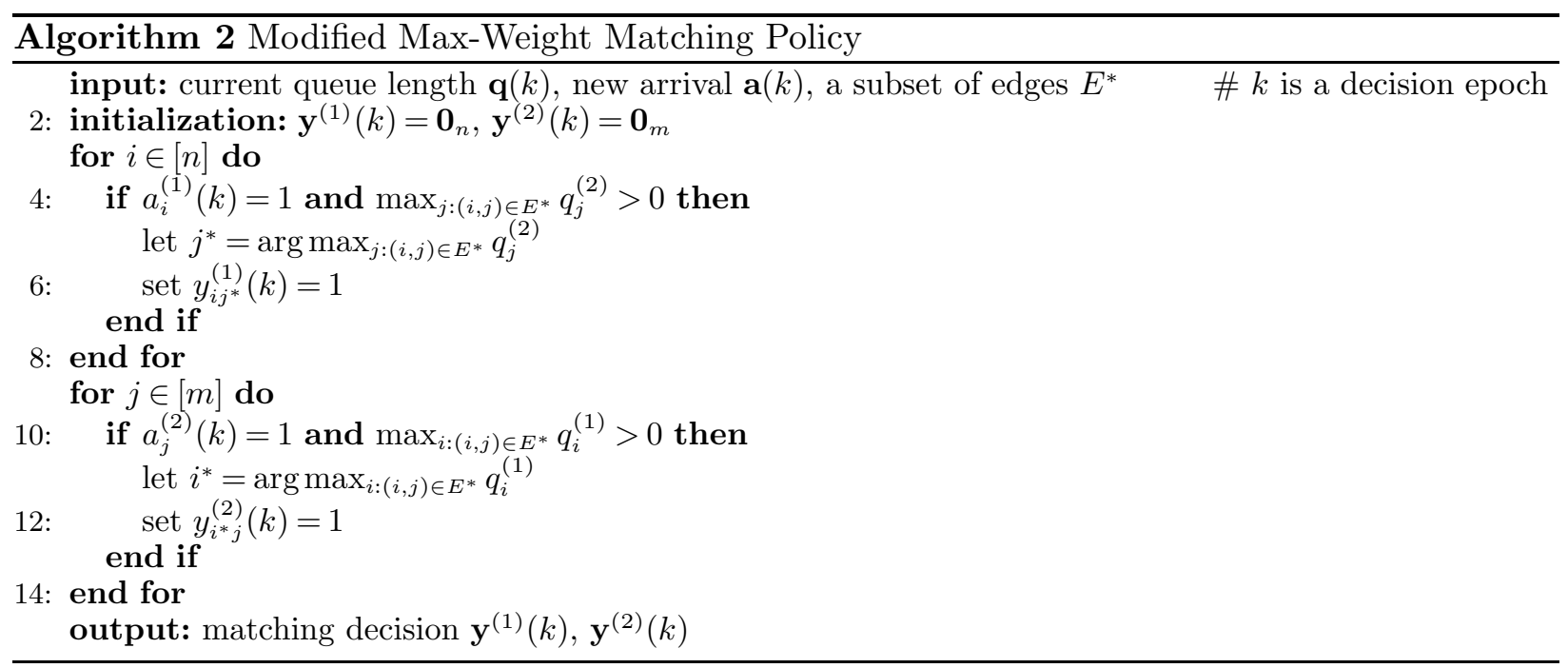

We present two lemmas that will help us prove Theorem 2. The first lemma shows that the continuous time Markov chain associated with two-price policy is positive recurrent, and furthermore gives a bound on the expected queue length.

Lemma 1. For a system of two-sided queues operating under the two-price policy and the modified max-weight matching algorithm parameterized by $\eta$, the system is positive recurrent for any $\boldsymbol{\theta}>\mathbf{0}_{m}$, $\phi>\mathbf{0}_{n}, \sigma^{\eta}>0$ and $\tau_{\max }^{\eta}>0$. The expected queue lengths are bounded by

$$
\begin{aligned}
\mathbb{E}\left[\left\langle\boldsymbol{\theta}, \mathbf{q}^{(2)}\right\rangle\right]+\mathbb{E}\left[\left\langle\boldsymbol{\phi}, \mathbf{q}^{(1)}\right\rangle\right] \leq & \tau_{\max }^{\eta}\left(\sum_{j=1}^{m} \theta_{j} \mathbb{P}\left[q_{j}^{(2)}>\tau_{\max }^{\eta}\right]+\sum_{i=1}^{n} \phi_{i} \mathbb{P}\left[q_{i}^{(1)}>\tau_{\max }^{\eta}\right]\right) \\
& +\frac{\eta}{\sigma^{\eta}}\left(\left\langle\mathbf{1}_{n}, \boldsymbol{\mu}^{*}\right\rangle+\left\langle\mathbf{1}_{m}, \boldsymbol{\lambda}^{*}\right\rangle\right) .
\end{aligned}
$$

Sketch Proof of Lemma 1. The complete proof of the lemma is presented in the Appendix F. We present a brief idea of the proof here. Since $\boldsymbol{\theta}, \boldsymbol{\phi}$ and $\sigma^{\eta}$ are greater than zero, when the queue length of a certain customer or server type is greater than its threshold, i.e. $\tau_{\max }^{\eta}$, the reduced arrival rate of that type leads to a drift towards 0 , so we expect that the system is positive recurrent. This intuition is proved rigorously using the Foster-Lyapunov Theorem (see e.g. Srikant and Ying|2014). We consider a quadratic Lyapunov function in the queue lengths, and show that the one-step drift is negative outside a finite set of states. We then use the moment bound theorem (Hajek 2006) to upper bound the expected queue length.

Now we know that under the two-price policy, all the members in the family of CTMCs are positive recurrent. This is only possible when the arrival rates satisfies the constraints in the fluid optimization problem (7b) (7c) (7d) . Below, we will present another lemma that will be later used to eliminate terms in the profit loss. 
Lemma 2. For a system of two-sided queues operating under two-price policy and max-weight matching policy, for any $\boldsymbol{\theta}>\mathbf{0}_{m}, \boldsymbol{\phi}>\mathbf{0}_{n}$ and $\tau_{\max }^{\eta}>0$, we have

$$
\sum_{j \in[m]}\left(F_{j}^{\prime}\left(\lambda_{j}^{*}\right) \lambda_{j}^{*}+F_{j}\left(\lambda_{j}^{*}\right)\right) \theta_{j} \mathbb{P}\left[q_{j}^{(2)}>\tau_{\max }^{\eta}\right]=\sum_{i \in[n]}\left(G_{i}^{\prime}\left(\mu_{i}^{*}\right) \mu_{i}^{*}+G_{i}\left(\mu_{i}^{*}\right)\right) \phi_{i} \mathbb{P}\left[q_{i}^{(1)}>\tau_{\max }^{\eta}\right] .
$$

The proof of the lemma is deferred to Appendix G due to space constraints. We will present a brief sketch of the proof here for better understanding of the reader.

Sketch Proof. We will use the fact that $\left(\boldsymbol{\lambda}^{*}, \boldsymbol{\mu}^{*}\right)$ is the optimal solution of the Fluid optimization problem. To prove this lemma, we will use the first order optimality condition on the fluid optimization problem which says that the directional derivative of the objective function along a feasible direction is zero. We will use the arrival rates of the two-price policy as another feasible point to find a feasible direction. Equating directional derivative to zero gives us the lemma.

We will now present the idea for the proof of Theorem 2 and defer the details to Appendix $\mathrm{H}$.

Idea of the Proof of Theorem 2 We will first upper bound the profit loss defined in (9), for the two-price policy using Taylor series expansion. We will then use Lemma 2 to eliminate first order terms from the profit loss and use Lemma 1 to bound the expected queue length. Finally, we will substitute $\tau_{\max }^{\eta}$ and $\sigma^{\eta}$ in terms of $\eta$ to get the result.

From the previous two sections, we can see that even a simple two-price dynamic pricing policy has a much better performance than a static (fluid) pricing policy. It is also practically easy to implement the two-price policy. This shows the advantage of dynamic price over static price.

\section{Lower Bound}

In this section, we will obtain lower bounds on the profit loss under a broad family of policies, and thus establish that the rates we obtain from two-price policy in Theorem 2 are optimal. In particular, we consider a family of pricing policies that have the following form:

$$
\begin{aligned}
& \lambda_{j}=\eta \lambda_{j}^{*}+f_{j}\left(\frac{\mathbf{q}}{\eta^{\alpha}}\right) \eta^{\beta} \quad \forall j \in[m], \\
& \mu_{i}=\eta \mu_{i}^{*}+g_{i}\left(\frac{\mathbf{q}}{\eta^{\alpha}}\right) \eta^{\beta} \quad \forall i \in[n] .
\end{aligned}
$$

The motivation for this choice is as follows. The first term in Eqs (12) and (13) (i.e., $\eta \lambda_{j}^{*}$ and $\eta \mu_{i}^{*}$ ) is static and is the solution of the fluid model; the second term accounts for dynamic adjustment as queue length changes. We assume the adjustment term can be further decomposed into two terms: a function that scales the queue length, $f_{j}(\cdot)$ or $g_{i}(\cdot)$, and a term that determines the scaling of price adjustments, $\eta^{\beta}$, for some $1>\beta>0$. Moreover, as the arrival rates are scaled up, the queue length will be asymptotically large. Thus, we also scale the queue length in function $f_{j}(\cdot)$ and $g_{i}(\cdot)$ for all $i \in[n]$ and $j \in[m]$ by $\eta^{\alpha}$ for some $1 \geq \alpha \geq 0$. Note that a similar family of pricing policies was studied by Kim and Randhawa (2017).

In addition, we require the pricing policy to satisfy the following conditions. 
CONDition 1. (a) There exists $\mathbf{M} \in \mathbb{R}_{+}^{m}$ and $\mathbf{N} \in \mathbb{R}_{+}^{n}$ such that $\left|f_{j}\left(\frac{\mathbf{q}}{\eta^{\alpha}}\right)\right| \leq M_{j}$ for all $j \in[m]$ and $\left|g_{i}\left(\frac{\mathbf{q}}{\eta^{\alpha}}\right)\right| \leq N_{i}$ for all $i \in[n]$ for all $\mathbf{q} \in S$ and for all $\eta \geq 1$.

(b) $0<\alpha+\beta \leq 1$.

(c) There exists $K>0$ and $\delta>0$ such that for all $j \in[m]$, if $\frac{q_{j}^{(2)}}{\eta^{\alpha}}>K$, then either $f_{j}\left(\frac{\mathrm{q}}{\eta^{\alpha}}\right)<-\delta$ or there exists $i:(i, j) \in E$ such that $g_{i}\left(\frac{\mathbf{q}}{\eta^{\alpha}}\right)>\delta$ for all $\eta$. Similarly for all $i \in[n]$, if $\frac{q_{i}^{(1)}}{\eta^{\alpha}}>K$, then either $g_{i}\left(\frac{\mathbf{q}}{\eta^{\alpha}}\right)<-\delta$ or there exists $j:(i, j) \in E$ such that $f_{j}\left(\frac{\mathbf{q}}{\eta^{\alpha}}\right)>\delta$ for all $\eta$.

Condition [1](a) requires the dynamic price adjustment term to be bounded (after appropriate scaling); this a technical assumption required for our analysis. Condition [1] (b)] states that the rate of scaling of the system state should be less than the rate of pricing policy converging to the fluid rates. (In the special case of single link operating under the two-price policy, this assumption is not needed; the result is presented later in this section.) Condition [1](c)] states that if queue length for type $j$ customer is large, we should either decrease the arrival rate of type $j$ customers or increase the arrival rate of any servers that can serve type $j$ customers. These conditions are fairly general, because the pricing function can depend on the complete system state (q), and we do not make any strong assumptions such as monotonicity, continuity or differentiablity on functions $f_{j}(\cdot)$ and $g_{i}(\cdot)$. Also, note that we do not require any assumption on the matching policy.

The two-price policy satisfies the above condition with

$$
f_{j}(\mathbf{q})=-\theta_{j} \mathbb{1}_{q_{j}^{(2)}>\tau_{\max }}(\forall j \in[m]), \quad g_{i}(\mathbf{q})=-\phi_{i} \mathbb{1}_{q_{i}^{(1)}>\tau_{\max }}(\forall i \in[n]), \quad \beta=2 / 3 .
$$

Now we present the result on the lower bound.

THEOREM 3. For a two-sided queue defined by a graph $G\left(N_{1} \cup N_{2}, E\right)$ operating under any pricing policy of the form (12) and (13) that satisfies Condition 1, if the resulting system is stable, there exists a constant $K(F, G, f, g)$ such that

$$
L^{\eta} \geq K \eta^{1 / 3} .
$$

The details of the proof are deferred to Appendix I. Although, we present a brief idea of the proof here for better understanding of the reader.

Idea of the Proof. First we define a variable which quantifies the imbalance between the number of customers and servers in the system, which is crucial in our analysis. Mathematically, we define

$$
z=\left\langle\mathbf{1}_{m}, \mathbf{q}^{(2)}\right\rangle-\left\langle\mathbf{1}_{n}, \mathbf{q}^{(1)}\right\rangle
$$

An important observation is that the transition of $z$ conditioned on $q$ is independent of the matching policy. We use this fact to couple our system with a birth and death process to lower bound $\mathbb{E}[|z|]$, 
which can be used to further lower bound the expected queue length $\mathbb{E}\left[\left\langle\mathbf{1}_{m+n}, \mathbf{q}\right\rangle\right]$. We also use this coupling to lower bound $\mathbb{E}\left[\sum_{i=1}^{n} g_{i}^{2}()+.\sum_{j=1}^{m} f_{j}^{2}().\right]$ which is a proxy for the loss of profit.

Next, we use the lower bound on $\mathbb{E}\left[\left\langle\mathbf{1}_{m+n}, \mathbf{q}\right\rangle\right]$ and $\mathbb{E}\left[\sum_{i=1}^{n} g_{i}^{2}()+.\sum_{j=1}^{m} f_{j}^{2}().\right]$ to lower bound the profit loss. In particular, we use Taylor's expansion and Claim 2 from the fluid model to analyze the first order terms. The dominating terms in the lower bounds are given by $\eta^{2 \beta-1}$ (loss in the profit) and $\eta^{1-\beta}$ (expected queue length). The choice of $\beta$ trades-off between the queue length and the loss in profit. By picking $\beta=2 / 3$, we get the best over all profit loss, which gives the lower bound.

We can further relax Condition [1](b) in the special case of single link operating under the twoprice policy. The result is stated below.

Proposition 4. For a family of single link two-sided queue parametrized by $\eta$, any two pricing policy given by (10) (111) with $\sigma^{\eta}=\eta^{\beta}$ for some $\beta<1$ and $\tau_{\max }^{\eta}=\eta^{\alpha}$ for some $\alpha \in \mathbb{R}_{+}$, will have $a$ profit loss $L^{\eta}$ at least $\Omega\left(\eta^{1 / 3}\right)$. The choice of $\tau_{\max }^{\eta}=\eta^{1 / 3}$ and $\sigma^{\eta}=\eta^{2 / 3}$ and any positive constants $\boldsymbol{\theta}$ and $\boldsymbol{\phi}$ provides the optimal profit loss $\Theta\left(\eta^{1 / 3}\right)$.

The proof of Proposition 4 has been deferred to Appendix.

\section{LP-based Approximation Algorithm}

The Bellman equation for the MDP defined in the Section 2 can be rewritten as an optimization problem (see e.g. Bertsekas 2007):

$$
\begin{array}{cl}
\min _{(\gamma, \mathbf{h})} & \gamma \\
\text { subject to } & \gamma \geq \mathcal{R}(\mathbf{q}, \mathbf{z})+c \mathbb{E}_{\mathbf{q}, \mathbf{z}}[V(h, \mathbf{q}, \mathbf{z})]-\operatorname{ch}(\mathbf{q}) \quad \forall \mathbf{q}, \mathbf{z} \in S \times Z(\mathbf{q}),
\end{array}
$$

where $\mathcal{R}(.,$.$) represents the revenue rate and is defined in \mathrm{Eq}(44)$, and $\mathbb{E}_{\mathbf{q}, \mathbf{z}}[V(h, \mathbf{q}, \mathbf{z})]$ is defined in $\mathrm{Eq}(\underline{5)}$.

It is difficult to solve this problem computationally, as we have one constraint associated with each state-action pair in (17). Due to the curse of dimensionality, the state space $S$ will increase exponentially with the customer and server types. Moreover, the action space $Z(\mathbf{q})$ contains continuous prices, so the action space is uncountable. Of course, one can discretize the action space, but a fine discretization may be needed to ensure numerical accuracy, which also increases the computation burden.

Our proposed approach is to reduce the number of decision variables in the optimization problem by restricting the bias function $\mathbf{h}(\mathbf{q})$ to some parametric form. This will lead to an optimization where the number of decision variables is polynomial in customer/server types. We can also use the original action space, so the discretization step for the action space is not needed.

Throughout this section, we assume the queue length is bounded, that is, $S \subseteq\left\{\mathbf{q}: \mathbf{q} \leq \mathbf{1}_{m+n} q_{\max }\right\}$ for some $q_{\max }<\infty$. 


\subsection{Polynomial Approximation}

Now, we will present the approximation of the bias function.

Proposition 5. Approximating the bias $\mathbf{h}(\mathbf{q})$ with a polynomial in $q$ of degree $r$ given by

$$
h(\mathbf{q})=\sum_{l=1}^{r}\left(\sum_{i=1}^{n} b_{l_{1}}^{(1)}\left(q_{i}^{(1)}\right)^{l}+\sum_{j=1}^{m} b_{l_{1}}^{(2)}\left(q_{j}^{(2)}\right)^{l}\right) \triangleq \sum_{l=1}^{r}\left\langle\mathbf{b}_{l}, \mathbf{q}^{l}\right\rangle,
$$

for some finite $r \in \mathbb{Z}_{+}$and solving the optimization problem (16), (17) gives an upper bound on the average revenue $g$. Here, $\mathbf{b}_{l}$ is the defined as the vector $\left(b_{l_{1}}^{(1)}, \ldots, b_{l_{n}}^{(1)}, b_{l_{1}}^{(2)}, \ldots, b_{l_{m}}^{(2)}\right)$ for all $l \in[r]$.

The proof is based on the analysis of the minimization problem to solve the MDP. It uses the idea that if we minimize over a subset of the feasible region, then it will lead to higher values of the optimal objective function value and is deferred to Appendix $\mathrm{K}$.

By approximating the bias by a polynomial of the queue length of degree $r$, we have reduced the number of variables in the optimization problem from $q_{\max }^{m+n}$ to just $(m+n) \times r$ which is linear in $m$ and $n$ if $r$ is a constant. We will later see that this approximation reduces the computational time drastically.

Also, as the degree of the polynomial increases, we consider a more general case, and thus the upper bound we obtain on the average revenue will be tighter as $r$ increases. The system operator can choose this $r$ to balance the trade-off between accuracy of the solution and the computational time.

Another useful feature of this approximation is that, if we fix a stationary matching policy $\mathbf{x}(\mathbf{q})$, then we can use the optimality equations for the Bellman equation (3) to compute the closed form expression of the approximated pricing policy by solving the following differential equations:

$$
\begin{aligned}
& \left(F\left(\lambda_{j}\right) \lambda_{j}\right)^{\prime}+h\left(\mathbf{q}+\mathbf{e}_{j}^{(2)}-\mathbf{x}\right)-h(\mathbf{q})=0 \forall j \in[m], \\
& \left(G\left(\mu_{i}\right) \mu_{i}\right)^{\prime}+h\left(\mathbf{q}+\mathbf{e}_{i}^{(1)}-\mathbf{x}\right)-h(\mathbf{q})=0 \forall i \in[n] .
\end{aligned}
$$

These optimality equations are only valid when we are strictly in the interior of the feasible region of the optimization problem, i.e. $\boldsymbol{\lambda}>\mathbf{0}_{m}$ and $\boldsymbol{\mu}>\mathbf{0}_{n}$. Although, if there exists a $q_{j_{0}}^{(2)}$ for which $\lambda_{j}\left(q_{j_{0}}^{(2)}\right)=0$ then we can restrict our state space to $q_{j}^{(2)} \leq q_{j_{0}}^{(2)}$ as the arrival rate of that type of customer for $q_{j_{0}}^{(2)}$ is 0 and thus, it is not possible to have $q_{j}^{(2)}>q_{j_{0}}^{(2)}$.

Note on Optimal Matching Policy: We will fix the pricing policy to the fluid pricing policy introduced in section 4 and focus on optimal matching policy. We can rewrite the Bellman equation as follows:

$$
\frac{\gamma}{c}=\frac{\mathcal{R}\left(\mathbf{q}, \boldsymbol{\lambda}^{*}, \boldsymbol{\mu}^{*}\right)}{c}+\max _{\mathbf{x} \in X(\mathbf{q})}\left\{\mathbb{E}_{\mathbf{q}, \mathbf{z}}[V(h, \mathbf{q}, \mathbf{z})]-h(\mathbf{q})\right\}, \quad \forall \mathbf{q} \in S \cap\left\{\mathbf{q}: \mathbf{q} \leq q_{\max } \mathbf{1}_{n+m}\right\} .
$$


The corresponding optimization problem to find the optimal matching policy becomes

$$
\mathbf{x}^{*}(\mathbf{q})=\underset{\mathbf{x} \in X(\mathbf{q})}{\arg \max }\left\{\mathbb{E}_{\mathbf{q}, \mathbf{z}}[V(h, \mathbf{q}, \mathbf{z})]-h(\mathbf{q})\right\} \forall \mathbf{q} \in S \cap\left\{\mathbf{q}: \mathbf{q} \leq q_{\max } \mathbf{1}_{n+m}\right\} .
$$

Here, we are greedily maximizing the expected difference of the bias function before and after (one step drift) one matching. Thus, the optimal matching policy is "max-weight" policy if the bias function prescribes weight to each state. The policy is optimal as the long term behaviour is captured by the bias function.

Now, if we approximate this bias function by some linear combination of basis vectors (e.g. polynomial function of $\mathbf{q}$ ), then we will get an approximately optimal matching policy. In particular, if we approximate the bias function by linear function of queue length, given by $h(\mathbf{q})=\langle\mathbf{b}, \mathbf{q}\rangle$ for some constant $\mathbf{b}$, then we get an open loop matching policy. This can be easily seen as follows:

$$
\begin{aligned}
\mathbf{x}^{*}(\mathbf{q}) & =\underset{\mathbf{x} \in X(\mathbf{q})}{\arg \max }\left\{\mathbb{E}_{\mathbf{q}, \mathbf{z}}[V(h, \mathbf{q}, \mathbf{z})]-h(\mathbf{q})\right\} \\
& =\underset{\mathbf{x} \in X(\mathbf{q})}{\arg \max }\left\{\sum_{j=1}^{m} \lambda_{j}^{*}\left(h\left(\mathbf{q}+\mathbf{e}_{j}^{(2)}-\mathbf{x}\right)-h(\mathbf{q})\right)+\sum_{i=1}^{n} \mu_{i}^{*}\left(h\left(\mathbf{q}+\mathbf{e}_{i}^{(1)}-\mathbf{x}\right)-h(\mathbf{q})\right)\right\} \\
& =\underset{\mathbf{x} \in X(\mathbf{q})}{\arg \max }\{\langle-\mathbf{b}, \mathbf{x}\rangle\} .
\end{aligned}
$$

Thus, it translates into max-weight with the weights being $-\mathbf{b}$ for the customers and servers. As these weights are independent of the state, it is an open loop policy.

Similarly, if we approximate the bias function by a quadratic function of queue lengths given by

$$
h(\mathbf{q})=-\sum_{j=1}^{m}\left(q_{j}^{(2)}\right)^{2}-\sum_{i=1}^{n}\left(q_{i}^{(1)}\right)^{2}
$$

then we get a policy similar to max-weight matching policy given in Algorithm 1. We have

$$
\begin{aligned}
\mathbf{x}^{*}(\mathbf{q})= & \underset{\mathbf{x} \in X(\mathbf{q})}{\arg \max }\left\{\sum_{j=1}^{m} \lambda_{j}^{*}\left(h\left(\mathbf{q}+\mathbf{e}_{j}^{(2)}-\mathbf{x}\right)-h(\mathbf{q})\right)+\sum_{i=1}^{n} \mu_{i}^{*}\left(h\left(\mathbf{q}+\mathbf{e}_{i}^{(1)}-\mathbf{x}\right)-h(\mathbf{q})\right)\right\} \\
= & \underset{\mathbf{x} \in X(\mathbf{q})}{\arg \max }\left\{-\sum_{j=1}^{m} \lambda_{j}^{*}\left(\sum_{j^{\prime}=1}^{m}\left(x_{j^{\prime}}^{(2)}\right)^{2}+2 \sum_{j^{\prime}=1}^{m} q_{j^{\prime}}^{(2)} x_{j^{\prime}}^{(2)}+2 x_{j}^{(2)}\right)+\right. \\
& \left.\sum_{i=1}^{n} \mu_{i}^{*}\left(-\sum_{i^{\prime}=1}^{n}\left(x_{i^{\prime}}^{(1)}\right)^{2}+2 \sum_{i^{\prime}=1}^{n} q_{i^{\prime}}^{(1)} x_{i^{\prime}}^{(1)}+2 x_{i}^{(1)}\right)\right\} \\
* & \underset{\mathbf{x} \in X(\mathbf{q})}{\arg \max }\left\{-\sum_{j=1}^{m}\left(x_{j}^{(2)}\right)^{2}-\sum_{i=1}^{n}\left(x_{i}^{(1)}\right)^{2}+2\left(\sum_{j=1}^{m}\left(q_{j}^{(2)}+\frac{\lambda_{j}^{*}}{\left\langle\mathbf{1}, \boldsymbol{\lambda}^{*}\right\rangle}\right) x_{j}^{(2)}\right.\right. \\
& \left.\left.+\sum_{i=1}^{n}\left(q_{i}^{(1)}+\frac{\mu_{i}^{*}}{\left\langle\mathbf{1}, \boldsymbol{\mu}^{*}\right\rangle}\right) x_{i}^{(1)}\right)\right\} \\
* & \stackrel{* *}{\arg \max }\left\{\left(\sum_{\mathbf{x} \in X(\mathbf{q})}^{m}\left(q_{j}^{(2)}+\frac{\lambda_{j}^{*}}{\left\langle\mathbf{1}, \boldsymbol{\lambda}^{*}\right\rangle}\right) x_{j}^{(2)}+\sum_{i=1}^{n}\left(q_{i}^{(1)}+\frac{\mu_{i}^{*}}{\left\langle\mathbf{1}, \boldsymbol{\mu}^{*}\right\rangle}\right) x_{i}^{(1)}\right)\right\} \approx \underset{\mathbf{x} \in X(\mathbf{q})}{\arg \max \{\langle\mathbf{q}, \mathbf{x}\rangle\},}
\end{aligned}
$$


where, $(*)$ follows as $\left\langle\mathbf{1}_{m}, \boldsymbol{\lambda}^{*}\right\rangle=\left\langle\mathbf{1}_{n}, \boldsymbol{\mu}^{*}\right\rangle$ and (**) follows as the objective function (19) is strictly positive for some $\mathbf{x} \in X(\mathbf{q})$ but it is zero for $\mathbf{x}=0$. Thus, it is always better to match instantaneously. So, with no batching, we have $\left\langle\mathbf{1}_{n+m}, \mathbf{x}^{2}\right\rangle$ is either 2 or 0 as in each decision epoch, there is one arrival and we always match if we can. Finally, the last approximation is motivated by the fact that it is difficult to accurately estimate the arrival rates of the customers and servers. Thus, we omit the dependency of the arrival rates from our policy. c

\subsection{Constraint Generation Algorithm}

In this section, we present the constraint generation technique to solve the optimization problem (16)-(17) after we have approximated the bias by a polynomial function in q. The constraint generation algorithm is as follows:

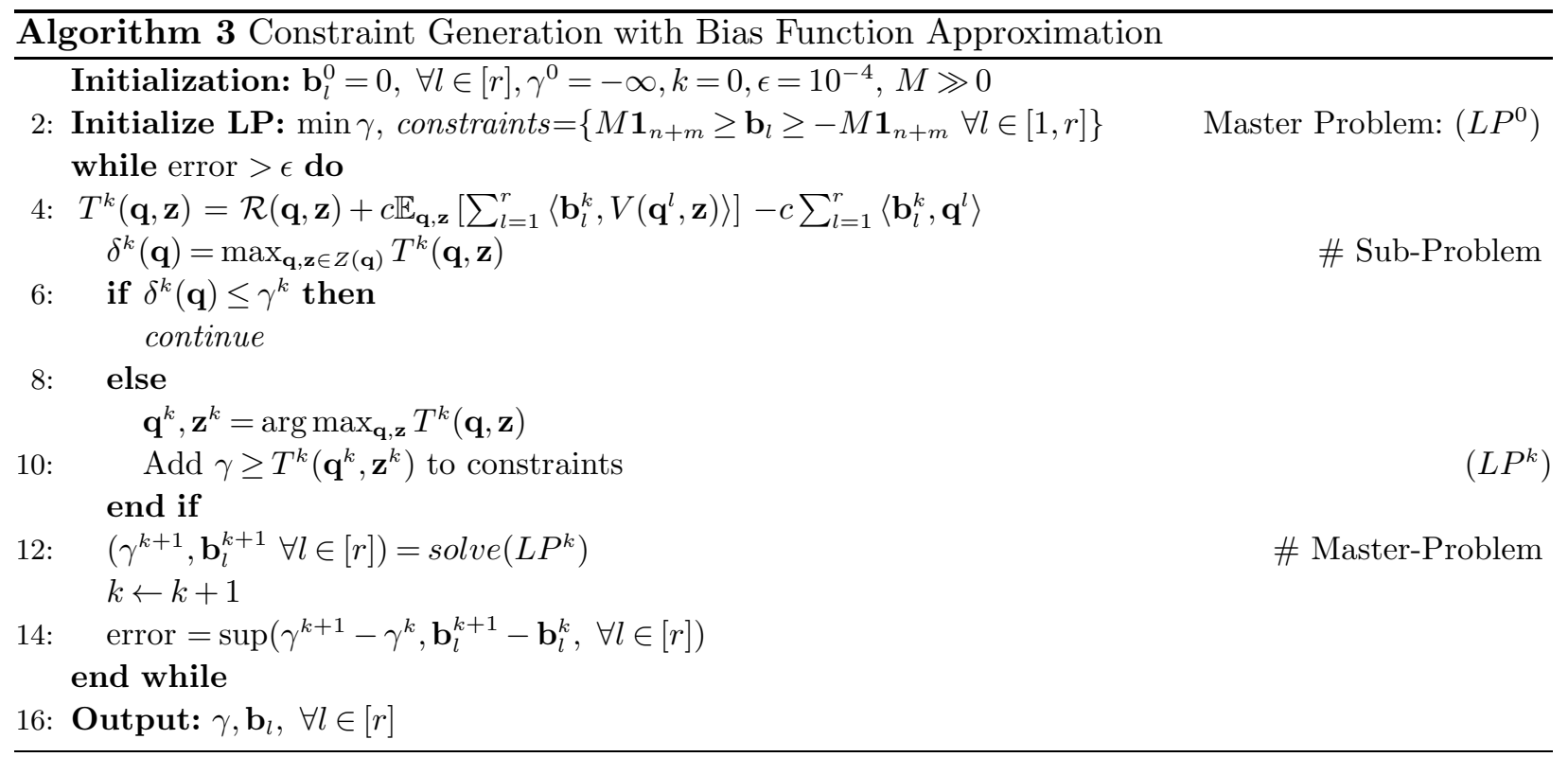

We initialize the variables $\left\{\mathbf{b}_{l}, l \in[r]\right\}$, which are the coefficients of the polynomial approximation of the bias function and the master LP with objective function "min $\gamma$ " and add an upper and lower bound on the decision variables $\mathbf{b}_{l} \forall l \in[1, r]$ in the constraints. This will make sure the iterates $\mathbf{b}_{l}^{k}$ are finite for all $k \in \mathbb{Z}_{+}$. In each iteration, we find the most violating constraint by solving the following sub-problem

$$
\max _{\mathbf{q} \leq q_{\max } \mathbf{1}_{m+n} \mathbf{z} \in Z(\mathbf{q})} \mathcal{R}(\mathbf{q}, \mathbf{z})+c \mathbb{E}_{\mathbf{q}, \mathbf{z}}\left[\sum_{l=1}^{r}\left\langle\mathbf{b}_{l}^{k}, V\left(\mathbf{q}^{l}, \mathbf{z}\right)\right\rangle\right]-c \sum_{l=1}^{r}\left\langle\mathbf{b}_{l}^{k}, \mathbf{q}^{l}\right\rangle .
$$


We then add these most violating constraints to the master-problem. We then solve the masterproblem to get the updated values of $\mathbf{b}_{l} \forall l \in[r]$ and $\gamma$. This process is repeated until the following criterion is met:

$$
\sup \left(\gamma^{k+1}-\gamma^{k}, \mathbf{b}_{l}^{k+1}-\mathbf{b}_{l}^{k} \forall l \in[r]\right) \leq \epsilon,
$$

where $k$ and $k+1$ are successive iterates obtained by repeating the constraint generation algorithm and $\epsilon$ is the allowable tolerance.

Sub-Problem The optimization problem can be solved employing the projected gradient descent or any similar method. A fixed number $(K)$ of iterations are performed for each sub-problem and that will give us a sub-optimal solution. If the constraint generated by this solution is feasible, then double the iterations $(2 K)$ of the projected gradient descent and continue. Otherwise, add this constraint generated to the master problem and solve the resultant LP to get updated parameters of the bias function. Now, we illustrate the sub-problem obtained by different approximations of the bias functions.

The linear approximation of the bias function results in the following optimization problem:

$$
\max _{\mathbf{q}, \mathbf{z}}\left\{\mathcal{R}(\mathbf{q}, \mathbf{z})+\left\langle\boldsymbol{\lambda}, \mathbf{b}_{1}^{(2)}\right\rangle+\left\langle\boldsymbol{\mu}, \mathbf{b}_{1}^{(1)}\right\rangle-\left(\left\langle\mathbf{1}_{m}, \boldsymbol{\lambda}\right\rangle+\left\langle\mathbf{1}_{n}, \boldsymbol{\mu}\right\rangle\right)\left\langle\mathbf{b}_{1}, \mathbf{x}\right\rangle\right\} \text { subject to (1) }
$$

We have a bi-linear term in $\boldsymbol{\lambda}+\boldsymbol{\mu}$ and $\mathbf{x}$. Although, using the constraint (1), we can lower bound $\left\langle\mathbf{b}_{1}, \mathbf{x}\right\rangle$ in terms of $\mathbf{b}_{1}$ and $\mathbf{q}$. This lower bound will hold tightly as we are maximizing with respect to $\mathbf{x}$. Let the lower bound be $l\left(\mathbf{b}_{1}, \mathbf{q}\right)$. We have

$$
\max _{\mathbf{q}, \mathbf{z}}\left\{\mathcal{R}(\mathbf{q}, \mathbf{z})+\left\langle\boldsymbol{\lambda}, \mathbf{b}_{1}^{(2)}\right\rangle+\left\langle\boldsymbol{\mu}, \mathbf{b}_{1}^{(1)}\right\rangle-\left(\left\langle\mathbf{1}_{m}, \boldsymbol{\lambda}\right\rangle+\left\langle\mathbf{1}_{n}, \boldsymbol{\mu}\right\rangle\right) l\left(\mathbf{b}_{1}, \mathbf{q}\right)\right\}
$$

Thus, we have an unconstrained maximization problem of sum of a concave function and a bi-linear term.

In addition, the $\mathbf{x}$ which achieves this lower bound for a given $\mathbf{q}$ corresponds to the max-weight matching with weights $-\mathbf{b}$ for the customers and servers. This can be easily seen as follows:

$$
l\left(\mathbf{b}_{1}, \mathbf{q}\right)=\max _{\mathbf{x}}\left\langle-\mathbf{b}_{1}, \mathbf{x}\right\rangle \text { subject to (1). }
$$

Thus, by solving the bellman equation with linear approximation will lead to max-weight matching policy and the output of the solution of the bellman equation is the weights of the max-weight matching. 


\section{Simulation}

In this section, we will present simulation results to verify the theorems presented before and to show the structural properties of the optimal pricing policies. We will only discuss the simulation results for a single link two-sided queue since it is easy to present the pricing policies for this case. We have also performed extensive tests for multi-link systems and found that they show similar insights, so we omit test results for multi-link systems.

For most of the results presented in this section, we use a supply curve given by $p_{1}=\lambda^{0.5}$ and a demand curve given by $p_{2}=4 \mu^{-0.5}$. Solving the fluid solution, we get the fluid optimal revenue of 3.08 , when $\lambda=\mu=\frac{4}{3}$ and $p_{1}=1.15$ and $p_{2}=3.46$. For most of the results, we cap the maximum queue length at 100 for both customers and servers as experiments pointed out that the expected queue length under optimal pricing policies is one order less than that.

\subsection{Optimal Pricing Policy}

We present the optimal pricing policy obtained by solving the Average Reward, Continuous Time Markov Decision Process using the Relative Value Iteration which gives us $\epsilon$ optimal solution where we specify the tolerance to be $10^{-4}$. The system is simulated for different values of the penalty coefficient of the waiting customers and servers. Fig. 4 show the optimal pricing policy for three different values of the penalty coefficient $(s)$. Note that, as $s$ increases queue length is penalized more and so the price increases more steeply as the number of customers and servers waiting in the system increases. Also note that the customer price is always above the server price and both of them are monotonic so that the platform makes a profit on an average. It verifies Proposition 1 . Also note that, when the system is empty, the customer and server price matches the fluid prices as there is no penalty due to the waiting and the revenue maximization problem is the fluid problem. As the system has more customers, the customer price is increased to reduce the inflow of the customers and server price is increased to increase the inflow of servers to balance out the waiting customers in the system. As $s$ increases, more weight is given to the penalty due to the waiting which leads to higher price in order to reduce the average number of waiting customers and servers.

The average revenue under the optimal pricing policies for different values of $s$ values is presented in Fig. 5. The average revenue decreases with the increase of $s$ as more penalty is imposed for each waiting customer and server. Fig. 4 and Fig. 6 show that as $s$ increases, the optimal pricing policy becomes steeper and the stationary distribution of the queue length is more concentrated around 0 . 
Figure 4 Optimal pricing policies under different values of penalty coefficients.

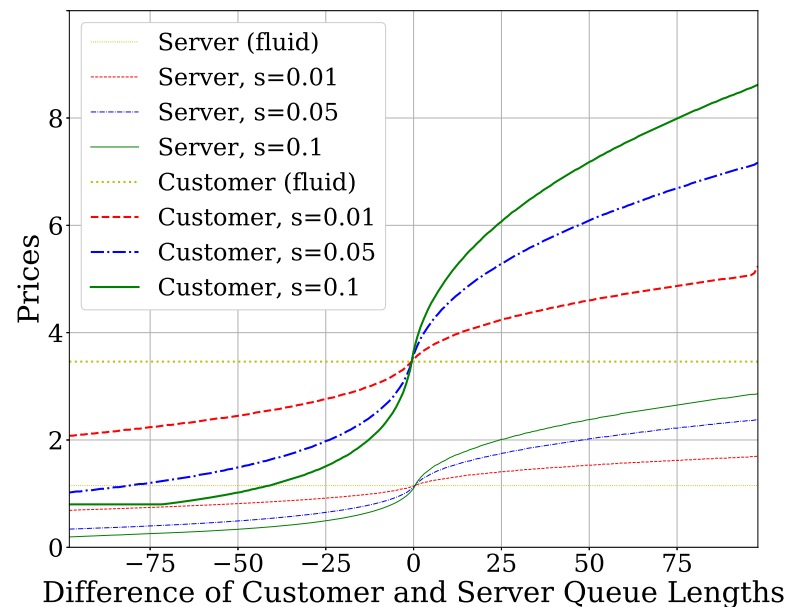

Figure 6 Stationary distribution of queue length under different penalty coefficients.

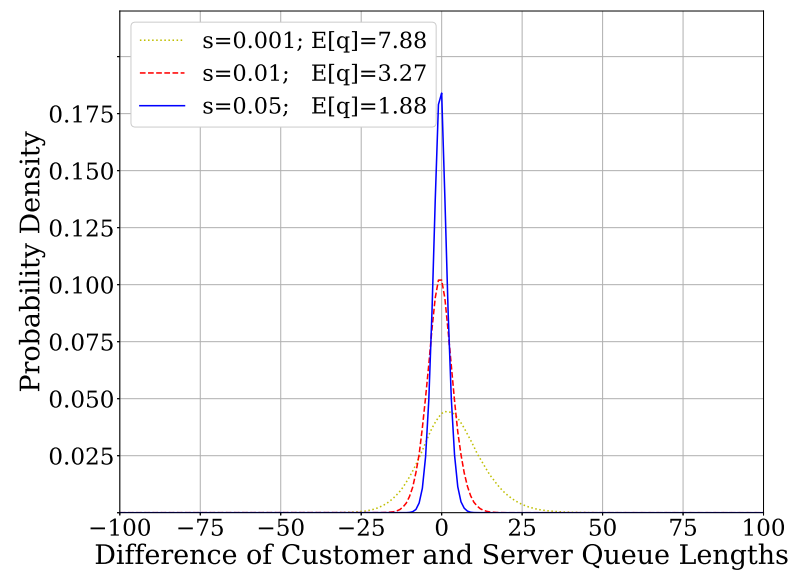

Figure 5 Optimal average profit obtained under different values of penalty coefficients.

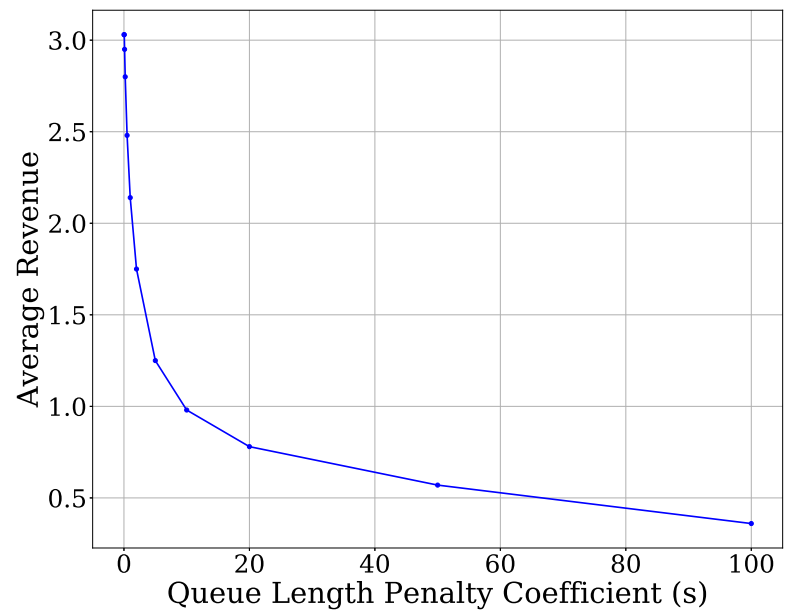

Figure 7 Comparison of pricing policies with bias approximation (penalty coefficient $=0.01$ ).

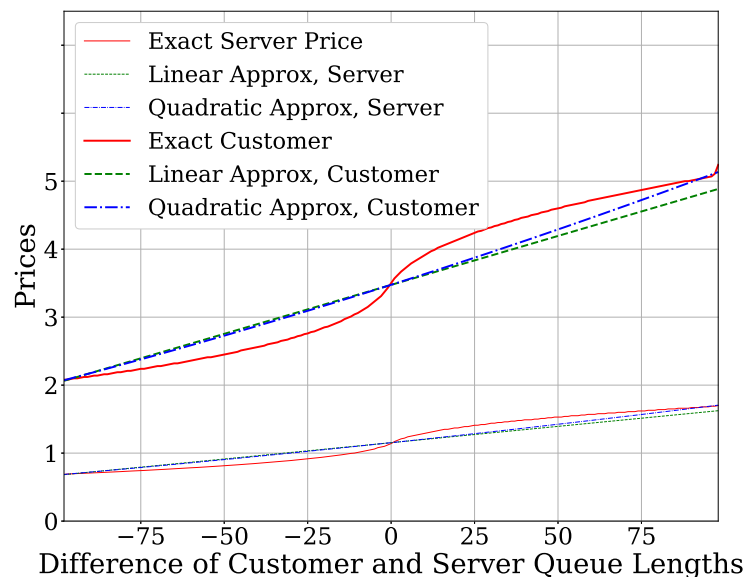

\subsection{Linear Approximation}

Now, we will present the results obtained by approximating the bias function by a polynomial in the queue length and solving the approximate Markov Decision Process using constraint generation. We would like to point out that the implementation of the approximate MDP using constraint generation takes 2 orders of time less than solving the MDP using relative value iteration. Among the multiple experiments conducted, relative value iteration took 5 minutes on an average. On the other hand, approximated MDP was solved under 10 seconds.

We will now compare the pricing policy obtained by linear and quadratic approximation of the bias function with that of the optimal pricing policy. As seen in Fig. 7 and 8 , for different values of $s$, the approximate pricing policy is a good first order approximation to the optimal pricing policy and with increasing the order of the polynomial, it results in a better fit. The linear approximation 
Figure 8 Comparison of pricing policies with bias approximation (penalty coefficient $=0.05$ ).

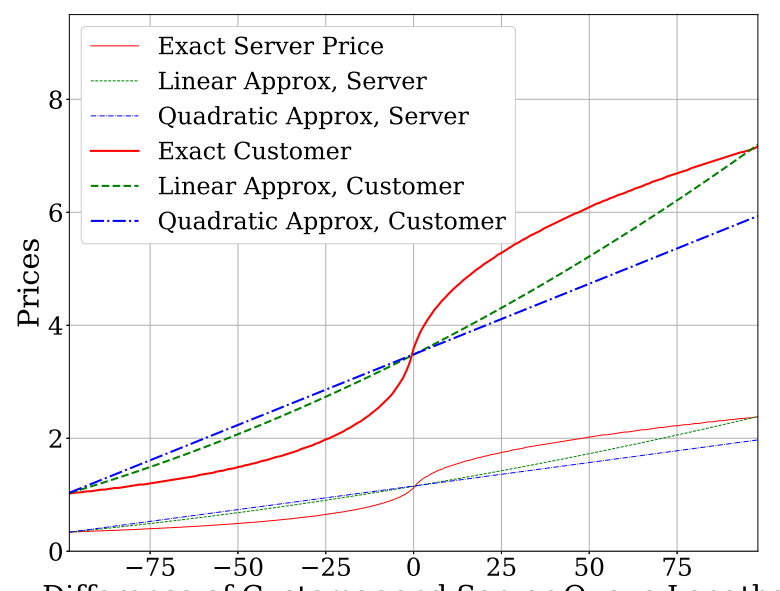

Figure 9 Performance of two-price policy and fluid pricing policy compared to the exact solution.

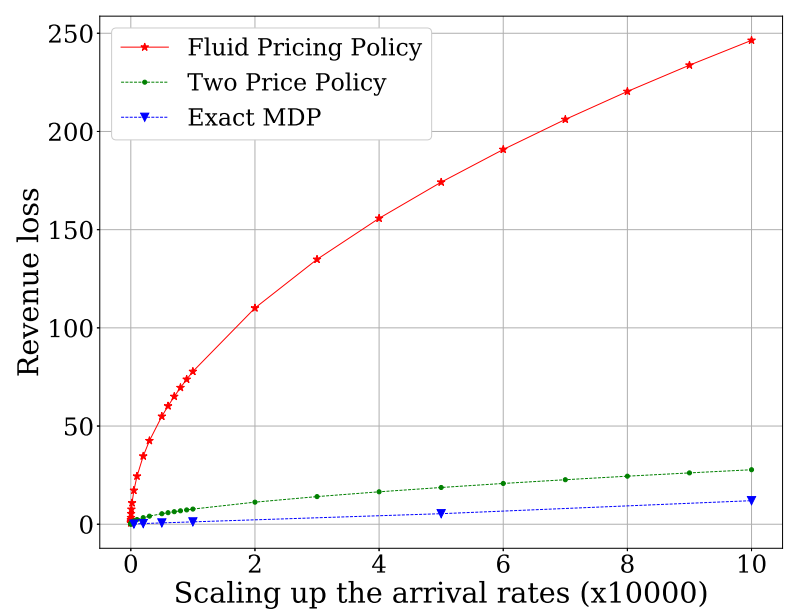

itself results in a way better fit than the fluid solution which gives only a static policy. We would also like to point out that by approximating the bias function and solving for the unknown coefficients, results in a closed form expression of the pricing policy. Although, the shape of the approximate policy is sensitive to the function used to approximate the bias function and the fit with the optimal pricing policy depends on the system parameters.

We compare the approximation of the bias function by a linear function in queue length with the exact bias function approximated as a linear function using regression. The comparison is summarized in Table 1. The bias function departs from a linear function to a more non linear function with increasing $s$ which leads to increasing mismatch between the exact and approximated bias function. Thus, the percentage error between the intercept and slope increases with the increasing value of $s$.

Moreover, we also compare the two by considering linear supply and demand curve. In particular, we consider the supply curve to be $p_{1}=\mu$ and $p_{2}=5-\lambda$. The comparison is summarized in Table 2. Observe that the percentage error is less in the case of linear supply and demand curve, which shows that the quality of the fit depends on the system parameters.

In both the cases, we can also see that the average revenue $(g)$ obtained by solving the approximated MDP results in an upper bound on the revenue obtained by solving the exact MDP. This verifies Proposition 5.

\subsection{Asymptotic Analysis}

We compute the profit loss under the fluid pricing policy and two-price policy and compare it with the theoretical result presented before and also with the exact solution obtained by solving 
Table 1 Comparison of constraint generation solution with the optimal solution with log-log supply and demand curves.

\begin{tabular}{c|ccc|ccc|cc}
\hline \multirow{2}{*}{$s$} & \multicolumn{2}{|c|}{ Cons. Generation } & \multicolumn{2}{c|}{ Value Iteration+Fit } & \multicolumn{2}{c}{ \% Error } \\
& $c_{0}$ & $c_{1}$ & $\gamma$ & $c_{0}$ & $c_{1}$ & $\gamma$ & $c_{0}$ & $c_{1}$ \\
\hline 0.01 & -1.73 & -0.007 & 3.07 & -1.69 & -0.008 & 3.03 & $2 \%$ & $10 \%$ \\
0.02 & -1.73 & -0.010 & 3.07 & -1.80 & -0.013 & 3.03 & $4 \%$ & $25 \%$ \\
0.05 & -1.73 & -0.013 & 3.06 & -1.89 & -0.019 & 3.03 & $9 \%$ & $34 \%$ \\
0.1 & -1.73 & -0.014 & 3.06 & -2.05 & -0.025 & 2.95 & $16 \%$ & $43 \%$ \\
0.2 & -1.73 & -0.016 & 3.06 & -2.59 & -0.034 & 2.8 & $24 \%$ & $53 \%$ \\
0.5 & -1.73 & -0.017 & 3.06 & -2.59 & -0.051 & 2.48 & $33 \%$ & $67 \%$ \\
\hline
\end{tabular}

Table 2 Comparison of constraint generation solution with the solution with linear supply and demand curves.

\begin{tabular}{c|ccc|ccc|cc}
\hline \multirow{2}{*}{$s$} & \multicolumn{2}{|c|}{ Cons. Generation } & \multicolumn{2}{|c|}{ Value Iteration+Fit } & \multicolumn{2}{c}{ \% Error } \\
& $c_{0}$ & $c_{1}$ & $\gamma$ & $c_{0}$ & $c_{1}$ & $\gamma$ & $c_{0}$ & $c_{1}$ \\
\hline 0.01 & -0.014 & -2.49 & 3.11 & -0.016 & -2.51 & 3.06 & $12 \%$ & $1 \%$ \\
0.02 & -0.020 & -2.49 & 3.10 & -0.023 & -2.51 & 3.02 & $13 \%$ & $1 \%$ \\
0.05 & -0.032 & -2.48 & 3.09 & -0.037 & -2.52 & 2.93 & $14 \%$ & $1 \%$ \\
0.1 & -0.048 & -2.48 & 3.07 & -0.054 & -2.53 & 2.81 & $12 \%$ & $2 \%$ \\
0.2 & -0.071 & -2.46 & 3.04 & -0.081 & -2.55 & 2.63 & $12 \%$ & $3 \%$ \\
0.5 & -0.121 & -2.44 & 2.98 & -0.144 & -2.58 & 2.24 & $16 \%$ & $5 \%$ \\
\hline
\end{tabular}

the MDP. The profit loss under the fluid pricing policy has an order of $\sqrt{\eta}$ and that under the two-price policy has an order of $\eta^{1 / 3}$, verifying Theorem 1 and Theorem 2 . Also observe that the profit loss under the two-price policy is not much different from that of the optimal profit loss, demonstrating the effectiveness of a two-price policy.

\section{Conclusion}

In this paper, we present a model of dynamic pricing and matching for two-sided queueing systems. The system is formulated as a continuous time Markov decision process, and a fluid approximation model is considered. We presented a fluid pricing and max-weight matching policy and showed that it achieves $O(\sqrt{\eta})$ optimality rate. Furthermore, we proposed a dynamic pricing and modified maxweight policy, which achieves $O\left(\eta^{1 / 3}\right)$ optimality rate. We also show that this scaling of $O\left(\eta^{1 / 3}\right)$ matches the lower bound for a broad family of policies. In addition, we presented an LP-based approximation framework and constraint generation algorithm. Our simulation results verify the theoretical findings and provide additional insights into the optimal policy structure.

\section{References}

Adan I, Weiss G (2012) Exact FCFS matching rates for two infinite multitype sequences. Operations Research 60(2):475-489.

Akbarpour M, Li S, Oveis Gharan S (2019) Thickness and information in dynamic matching markets. Journal of Political Economy (forthcoming). 
Anderson R, Ashlagi I, Gamarnik D, Kanoria Y (2017) Efficient dynamic barter exchange. Operations Research 65(6):1446-1459.

Banerjee S, Freund D, Lykouris T (2017) Pricing and optimization in shared vehicle systems: An approximation framework. Proceedings of the 2017 ACM Conference on Economics and Computation, 517-517.

Banerjee S, Johari R, Riquelme C (2016) Dynamic pricing in ridesharing platforms. ACM SIGecom Exchanges $15(1): 65-70$.

Banerjee S, Kanoria Y, Qian P (2018) State dependent control of closed queueing networks. ACM SIGMETRICS Performance Evaluation Review 46(1):2-4.

Bertsekas DP (2007) Dynamic Programming and Optimal Control, volume II (Athena Scientific), 4th edition.

Besbes O, Castro F, Lobel I (2018) Surge pricing and its spatial supply response. Columbia Business School Research Paper .

Caldentey R, Kaplan EH, Weiss G (2009) FCFS infinite bipartite matching of servers and customers. Advances in Applied Probability 41(3):695-730.

Chen H, Frank MZ (2001) State dependent pricing with a queue. IIE Transactions 33(10):847-860.

Chen Y, Hu M (2019) Pricing and matching with forward-looking buyers and sellers. Manufacturing \& Service Operations Management .

Gurvich I, Ward A (2014) On the dynamic control of matching queues. Stochastic Systems 4(2):479-523.

Hajek B (2006) Lecture notes for ECE 467 communication network analysis. URL http://www.ifp.illinois.edu/ hajek/Papers/networkanalysisDec06.pdf.

Hu B, Hu M, Zhu H (2019) Surge pricing and two-sided temporal responses in ride-hailing. Available at SSRN 3278023 .

Hu M, Zhou Y (2018) Dynamic type matching, Rotman School of Management Working Paper No. 2592622.

Kanoria Y, Qian P (2019) Near optimal control of a ride-hailing platform via mirror backpressure. arXiv preprint arXiv:1903.02764.

Kim J, Randhawa RS (2017) The value of dynamic pricing in large queueing systems. Operations Research 66(2):409-425.

Korolko N, Woodard D, Yan C, Zhu H (2018) Dynamic pricing and matching in ride-hailing platforms. Available at SSRN .

Low DW (1974a) Optimal dynamic pricing policies for an m/m/s queue. Operations Research 22(3):545-561.

Low DW (1974b) Optimal pricing for an unbounded queue. IBM Journal of research and Development 18(4):290-302.

Maguluri ST, Srikant R (2016) Heavy traffic queue length behavior in a switch under the MaxWeight algorithm. Stochastic Systems 6(1):211-250. 
Nguyen LM, Stolyar AL (2018) A queueing system with on-demand servers: local stability of fluid limits. Queueing Systems 89(3-4):243-268.

Paschalidis IC, Tsitsiklis JN (2000) Congestion-dependent pricing of network services. IEEE/ACM Transactions on networking 8(2):171-184.

Puterman ML (1994) Markov Decision Processes: Discrete Stochastic Dynamic Programming (New York, NY, USA: John Wiley \& Sons, Inc.).

Roth AE, Sönmez T, Ünver MU (2007) Efficient kidney exchange: Coincidence of wants in markets with compatibility-based preferences. American Economic Review 97(3):828-851.

Sivaraman V, Venkatakrishnan SB, Ruan K, Negi P, Yang L, Mittal R, Alizadeh M, Fanti G (2018) High throughput cryptocurrency routing in payment channel networks.

Srikant R, Ying L (2014) Communication Networks: An Optimization, Control and Stochastic Networks Perspective (New York, NY, USA: Cambridge University Press).

Talluri KT, Van Ryzin GJ (2006) The theory and practice of revenue management (Boston, MA, USA: Springer Science \& Business Media).

Tan B, Srikant R (2012) Online advertisement, optimization and stochastic networks. IEEE Transactions on Automatic Control 57(11):2854-2868.

Tassiulas L, Ephremides A (1992) Stability properties of constrained queueing systems and scheduling policies for maximum throughput in multihop radio networks. IEEE Transactions on Automatic Control 37(12):1936-1948. 


\section{Appendix}

\section{A. Uniformized Discrete Time MDP}

We define an arrival and matching process for the UDT-MDP. Let $\mathbf{a}(k) \in\{0,1\}^{m+n}$ be an arrival process, which represents the arrival of the customers and servers as we have one transition in the uniformized process. In each period of UDT-MDP, there is at most one customer or server arrival. The arriving probability is given by

As the probability of having multiple arrivals at the same time for a continuous time process is zero, at most one component of $\mathbf{a}(k)$ will be non zero. Thus, the arrival process has the following distribution:

$$
\mathbf{a}(k)= \begin{cases}\mathbf{e}_{j}^{(2)} & \text { w.p. } \frac{\lambda_{j}(\mathbf{q}(k))}{c}, \forall j \in[m] \\ \mathbf{e}_{i}^{(1)} & \text { w.p. } \frac{\mu_{i}(\mathbf{q}(k))}{c}, \forall i \in[n] \\ \mathbf{0}_{m+n} & \text { w.p. } 1-\frac{\left\langle\mathbf{1}_{m}, \boldsymbol{\lambda}(\mathbf{q}(k))\right\rangle+\left\langle\mathbf{1}_{n}, \boldsymbol{\mu}(\mathbf{q}(k))\right\rangle}{c} .\end{cases}
$$

Every time the system state changes, the operator is allowed to match customers and servers. Thus, we define the service process $\tilde{\mathbf{x}}$ as follows:

$$
\tilde{\mathbf{x}}(k)= \begin{cases}\mathbf{x}(\mathbf{q}(k)+\mathbf{a}(k)) & \text { if } \mathbf{a}(k) \neq \mathbf{0}_{m+n} \\ \mathbf{0} & \text { if } \mathbf{a}(k)=\mathbf{0}_{m+n},\end{cases}
$$

where $q(k)$ is queue length of the system just after we perform matching for the $(k-1)^{t h}$ decision epoch. With an abuse of notation, we use $\mathbf{x}(k)$ to represent $\mathbf{x}(\mathbf{q}(k)+\mathbf{a}(k))$. Now we have defined an arrival process and a service process ( matching decisions) of the two-sided queuing system. For the ease of notation, we define vectors of the queue lengths, arrival and service process as

$$
\mathbf{q}=\left(\begin{array}{c}
q_{1}^{(1)} \\
q_{2}^{(1)} \\
\vdots \\
q_{n}^{(1)} \\
q_{1}^{(2)} \\
q_{2}^{(2)} \\
\vdots \\
q_{m}^{(2)}
\end{array}\right) \quad \mathbf{a}=\left(\begin{array}{c}
a_{1}^{(1)} \\
a_{2}^{(1)} \\
\vdots \\
a_{n}^{(1)} \\
a_{1}^{(2)} \\
a_{2}^{(2)} \\
\vdots \\
a_{m}^{(2)}
\end{array}\right) \quad \tilde{\mathbf{x}}=\left(\begin{array}{c}
\tilde{x}_{1}^{(1)} \\
\tilde{x}_{2}^{(1)} \\
\vdots \\
\tilde{x}_{n}^{(1)} \\
\tilde{x}_{1}^{(2)} \\
\tilde{x}_{2}^{(2)} \\
\vdots \\
\tilde{x}_{m}^{(2)}
\end{array}\right)
$$

The queue length advancement equation is the relation between the queue length before a transition $\mathbf{q}(k)$ and the queue length after a transition $\mathbf{q}(k+1)$ in the UDT-MDP. We have

$$
\mathbf{q}(k+1)=\mathbf{q}(k)-\tilde{\mathbf{x}}(k)+\mathbf{a}(k) .
$$

Rather than keeping track of the trajectory of the queue length of the continuous process, it suffices to keep track of the queue length for the uniformized discrete time process. We will work with this discrete time process in the following sections. 


\section{B. Proof of Proposition 1}

The optimal customer and server pricing policy is obtained by solving the Bellman equation given by (6).

We will first show that the difference of the optimal bias function, $\Delta h(q)=h(q)-h(q-1)$ is monotonically decreasing in $q$. We will use the relative value iteration algorithm to compute the bias function and show that in every iteration, the bias function is monotonic where the bias function at the end of $k^{\text {th }}$ iteration is denoted by $\mathbf{h}_{k}(q)$. The relative value iteration does the following computation for all $q \in S$ :

$$
\begin{aligned}
h_{k+1}(q)=\max _{\mu \geq 0, \lambda \geq 0}\left[\frac{F(\lambda) \lambda-G(\mu) \mu}{c}-\frac{s|q|}{c}-\frac{\gamma}{c}+\frac{\lambda}{c} h_{k}(q+1)+\left(1-\frac{\mu+\lambda}{c}\right) h_{k}(q)\right. \\
\left.+\frac{\mu}{c} h_{k}(q-1)\right]-h_{k}(\tilde{q})
\end{aligned}
$$

for some $\tilde{q} \in S$. Also, the Bellman equation can be rewritten using $\Delta h(q)$ as follows:

$$
\gamma=\max _{\mu \geq 0, \lambda \geq 0}[F(\lambda) \lambda-G(\mu) \mu-s|q|+\lambda \Delta h(q+1)-\mu \Delta h(q)] \forall q \in S .
$$

Now we will first present a lemma which is essential to prove Proposition 1.

Lemma 3. The optimal difference of bias function $\Delta h^{*}(q)$ is monotonically decreasing in $q$.

Proof The proof by induction is presented below.

Base Case: As we can start with any initial bias function to implement relative value iteration algorithm, we pick a monotonically decreasing difference of bias function $\Delta \mathbf{h}_{0}(q)$ in $q$.

Induction Hypothesis: Assume that $\Delta \mathbf{h}_{k}(q)$ is monotonically decreasing in $q$.

Induction Step: We will now calculate $\Delta h_{k+1}(q+2)-\Delta h_{k+1}(q+1)$ and show that it is always non negative. We have,

$$
\begin{aligned}
& c \Delta h_{k+1}(q+2)-c \Delta h_{k+1}(q+1) \\
= & c\left(h_{k+1}(q+2)-2 h_{k+1}(q+1)+h_{k+1}(q)\right) \\
\stackrel{*}{*} & \Delta h_{k}(q+2)-c \Delta h_{k}(q+1) \\
& +\left\{\lambda^{*}(q+2) \Delta h_{k}(q+3)-\mu^{*}(q+2) \Delta h_{k}(q+2)+\mathcal{R}\left(\mu^{*}(q+2), \lambda^{*}(q+2)\right)\right\} \\
& -2\left\{\lambda^{*}(q+1) \Delta h_{k}(q+2)-\mu^{*}(q+1) \Delta h_{k}(q+1)+\mathcal{R}\left(\mu^{*}(q+1), \lambda^{*}(q+1)\right)\right\} \\
& +\left\{\lambda^{*}(q) \Delta h_{k}(q+1)-\mu^{*}(q) \Delta h_{k}(q)+\mathcal{R}\left(\mu^{*}(q), \lambda^{*}(q)\right)\right\},
\end{aligned}
$$

where $(*)$ follows from the Bellman equation where $\mathcal{R}\left(\mu^{*}(q), \lambda^{*}(q)\right)$ is the reward given by $F(\lambda) \lambda-$ $G(\mu) \mu-s|q|$ and $\left(\lambda^{*}\left(q_{0}\right), \mu^{*}\left(q_{0}\right)\right)$ maximizes the Bellman equation ([6) for $q=q_{0}$. As $\left(\lambda^{*}\left(q_{0}\right), \mu^{*}\left(q_{0}\right)\right)$ maximizes (6) for $q=q_{0}$, we have

$$
\begin{gathered}
\mathcal{R}\left(\mu^{*}(q+1), \lambda^{*}(q+1)\right)+\lambda^{*}(q+1) \Delta h_{k}(q+2)-\mu^{*}(q+1) \Delta h_{k}(q+1) \geq \mathcal{R}\left(\mu^{*}(q+i), \lambda^{*}(q+i)\right) \\
+\lambda^{*}(q+i) \Delta h_{k}(q+2)-\mu^{*}(q+i) \Delta h_{k}(q+1) \forall i \in\{0,2\} .
\end{gathered}
$$


Using, (24) to simplify (23) we get,

$$
\begin{gathered}
c \Delta h_{k+1}(q+2)-c \Delta h_{k+1}(q+1) \leq \lambda^{*}(q+2)\left(\Delta h_{k}(q+3)-\Delta h_{k}(q+2)\right)+\left(c-\mu^{*}(q+2)-\lambda^{*}(q)\right) \times \\
\left(\Delta h_{k}(q+2)-\Delta h_{k}(q+1)\right)+\mu^{*}(q)\left(\Delta h_{k}(q+1)-\Delta h_{k}(q)\right) \stackrel{*}{\leq} 0 \quad \forall q \in S
\end{gathered}
$$

As $c=\lambda_{\max }+\mu_{\max }$, we have $c-\mu^{*}(q+2)-\lambda^{*}(q) \geq 0$ for all $q$ and by Induction hypothesis, $\Delta h_{k}(q+1)-\Delta h_{k}(q) \leq 0$ for all $q$. Thus, $(*)$ follows. This proves the lemma as relative value iteration preserves the monotonic behaviour of the bias function and it converges to the optimal value and as the point-wise limit of monotonically decreasing functions is monotonically decreasing, $\Delta h(q)$ is monotonically decreasing and so $h(q)$ is concave.

Proof of Proposition 1 By Assumption 2, the domain of $F$ is $\left[0, \lambda_{\max }\right]$ and the domain of $G$ is $\left[0, \mu_{\max }\right]$ and by Assumption 1, the equation we are maximizing in (6) is continuous and by Assumption 2 is it concave. Thus, we are maximizing a continuous concave function over a compact set. Thus, the maximizer exists and any point is a maximizer if and only if it satisfies the first order necessary condition.

First we will show that if $\lambda^{*}\left(q_{0}\right)=0$ then $\lambda^{*}(q)=0$ for all $q>q_{0}$ is an optimal decision rule. If $\lambda^{*}\left(q_{0}\right)=0$, then we have

$$
\begin{gathered}
F(\lambda) \lambda+\Delta h^{*}\left(q_{0}\right) \lambda \leq 0 \forall \lambda \in\left[0, \lambda_{\max }\right] \\
F(\lambda) \lambda+\Delta h^{*}\left(q_{0}\right) \lambda+\left(\Delta h^{*}\left(q_{0}+k\right)-\Delta h^{*}\left(q_{0}\right)\right) \lambda \leq\left(\Delta h^{*}\left(q_{0}+k\right)-\Delta h^{*}\left(q_{0}\right)\right) \lambda \\
F(\lambda) \lambda+\Delta h^{*}\left(q_{0}+k\right) \lambda \stackrel{*}{\leq} 0 \forall \lambda \in\left[0, \lambda_{\max }\right] \forall k \geq 1
\end{gathered}
$$

where $(*)$ follows as $\Delta h^{*}$ is monotonically decreasing in $q$. The above inequality says that maximum value of $F(\lambda) \lambda+\Delta h^{*}\left(q_{0}+k\right) \lambda$ is zero for all $k \geq 1$, thus, $\lambda=0$ is an optimizer for that. Now we will similarly show that if $\lambda\left(q_{0}\right)=\lambda_{\max }$ then $\lambda(q)=\lambda_{\max }$ for all $q<q_{0}$ is an optimal decision rule. We have,

$$
F(\lambda) \lambda+\Delta h^{*}\left(q_{0}\right) \lambda \leq F\left(\lambda_{\max }\right) \lambda_{\max }+\Delta h^{*}\left(q_{0}\right) \lambda_{\max } \forall \lambda \in\left[0, \lambda_{\max }\right]
$$

Now, adding $\left(\Delta h^{*}\left(q_{0}-k\right)-\Delta h^{*}\left(q_{0}\right)\right) \lambda$ for some $k>0$, for all $\lambda \in\left[0, \lambda_{\max }\right]$ and $k \geq 1$, we get

$$
\begin{aligned}
& F(\lambda) \lambda+\Delta h^{*}\left(q_{0}-k\right) \lambda \leq F\left(\lambda_{\max }\right) \lambda_{\max }+\Delta h^{*}\left(q_{0}\right) \lambda_{\max }+\left(\Delta h^{*}\left(q_{0}-k\right)-\Delta h^{*}\left(q_{0}\right)\right) \lambda \\
\stackrel{*}{\Rightarrow} & F(\lambda) \lambda+\Delta h^{*}\left(q_{0}-k\right) \lambda \leq F\left(\lambda_{\max }\right) \lambda_{\max }+\Delta h^{*}\left(q_{0}-k\right) \lambda_{\max },
\end{aligned}
$$

where $(*)$ follows as $\lambda_{\max } \geq \lambda$ and $\Delta h^{*}\left(q_{0}-k\right)-\Delta h^{*}\left(q_{0}\right) \geq 0$. Thus, $\lambda(q)=\lambda_{\max }$ will be an optimal decision rule for all $q<q_{0}$. Thus, once the customer rate reaches it's maximum or minimum value, in the maximization problem ([6), it'll stay constantly that throughout. 
Similarly, we can show that if $\mu^{*}\left(q_{0}\right)=\mu_{\max }$ then $\mu(q)=\mu_{\max }$ for all $q \geq q_{0}$ is an optimal pricing policy and if $\mu^{*}\left(q_{0}\right)=0$ then $\mu(q)=0$ for all $q \leq q_{0}$ is an optimal pricing policy. As it is quite repetitive, we omit the details here.

Now, we will use the optimality condition for the optimization problem given by (6) when the optimal arrival rates are strictly in the interior of the constraint region, i.e. $\lambda_{\max }>\lambda^{*}(q)>0$ and $\mu_{\max }>\mu^{*}(q)>0$. We have,

$$
\begin{array}{r}
{\left[F\left(\lambda^{*}(q)\right) \lambda^{*}(q)\right]^{\prime}+\Delta h^{*}(q+1)=0} \\
{\left[G\left(\mu^{*}(q)\right) \mu^{*}(q)\right]^{\prime}+\Delta h^{*}(q)=0,}
\end{array}
$$

where the gradient of the LHS is taken with respect to $\lambda$ and $\mu$ respectively, which in turn depends on $q$. The gradient is well defined as $F$ and $G$ are continuous differentiable functions by Assumption 1. As $\Delta h^{*}(q)$ is monotonically decreasing in $q$ by Lemma 3, we have that $\left[F\left(\lambda^{*}(q)\right) \lambda^{*}(q)\right]^{\prime}$ and $\left[G\left(\mu^{*}(q)\right) \mu^{*}(q)\right]^{\prime}$ are monotonically increasing. By Assumption 2, $F\left(\lambda^{*}(q)\right) \lambda^{*}(q)$ is concave in $\lambda^{*}$ and $G\left(\mu^{*}(q)\right) \mu^{*}(q)$ is convex in $\mu^{*}$. Thus, $\lambda^{*}$ should be monotonically decreasing in $q$ and $\mu^{*}$ should be monotonically increasing in $q$ when $\lambda_{\max }>\lambda^{*}>0$ and $\mu_{\max }>\mu^{*}>0$ respectively.

Combining everything, $\lambda^{*}(q)$ will be monotonically decreasing for all $q$ and as the demand curve is monotonically decreasing by Assumption 1, the customer price $\left(p^{(2)}\right)^{*}(q)$ will be monotonically increasing. Also, $\mu^{*}(q)$ will be monotonically increasing for all $q$ and as the supply curve is monotonically increasing by Assumption 1, the server price $\left(p^{(1)}\right)^{*}(q)$ will be monotonically increasing.

\section{Proof of Proposition 2}

First note that, under a given pricing and matching policy, if $\mathbb{E}\left[q_{j}^{(k)}\right]=\infty$ for some $k, j \in\{1\} \times[m] \cup$ $\{2\} \times[n]$, then $\mathcal{R}(\mathbf{q}, \mathbf{z})=-\infty$ and the theorem is trivially true as the optimal objective function value (7a) is greater than or equal to 0 as $\tilde{\boldsymbol{\lambda}}=\mathbf{0}_{m}$ and $\tilde{\boldsymbol{\mu}}=\mathbf{0}_{n}$ is a trivial feasible solution of the fluid optimization problem.

We will now show the following claim holds true and then prove the theorem.

Claim 1. For any stationary pricing and matching policy under which the system is stable and $\mathbb{E}\left[q_{j}^{(k)}\right]<\infty$ for all $k, j \in\{1\} \times[m] \cup\{2\} \times[n]$, the constraints in the fluid $L P$ (7b) (7c) (77d) are necessary but not sufficient conditions.

Proof Recall that we are interested in policies under which our CTMC is irreducible containing the state zero. Thus, if under a pricing and matching policy the system is stable, then we can use stationarity to write:

$$
\mathbb{E}[\mathbf{q}(k+1)]=\mathbb{E}[\mathbf{q}(k)]
$$


where the expectation is with respect to the stationary distribution of the CTMC or equivalently the uniformized DTMC. Now, using (22), we can simplify the above equation to write:

$$
\mathbb{E}[\mathbf{a}(k)]=\mathbb{E}[\tilde{\mathbf{x}}(k)]
$$

We have $c \mathbb{E}[\mathbf{a}(k)]=c \mathbb{E}\left[\mathbb{E}_{\mathbf{q}(k)}[\mathbf{a}(k)]\right]=\mathbb{E}[(\boldsymbol{\lambda}(\mathbf{q}), \boldsymbol{\mu}(\mathbf{q}))]=(\tilde{\boldsymbol{\lambda}}, \tilde{\boldsymbol{\mu}})$, where $\boldsymbol{\lambda}(\mathbf{q})$ and $\boldsymbol{\mu}(\mathbf{q})$ are the arrival rates under the given pricing policy. By (1a $)$ (1b) and (1c), there exists $x_{i j}(k) \geq 0$ for all $i \in[n]$ and $j \in[m]$ and $k \in \mathbb{Z}_{+}$such that,

$$
\begin{aligned}
& \tilde{x}_{j}^{(2)}(k)=\sum_{i=1}^{n} \tilde{x}_{i j}(k) \leq q_{j}^{(2)}(k) \forall j \in[m], \\
& \tilde{x}_{i}^{(1)}(k)=\sum_{j=1}^{m} \tilde{x}_{i j}(k) \leq q_{i}^{(1)}(k) \forall i \in[n], \\
& \tilde{x}_{i j}(k)=0 \forall(i, j) \notin E,
\end{aligned}
$$

where $\tilde{x}_{i j}=x_{i j}$ if $\mathbf{a}(k) \neq \mathbf{0}_{m+n}$ and 0 otherwise. Note that since the matching policy is stationary, the expectation of the matching decision will not depend on $k$. Taking expectation on both sides with respect to the stationary distribution and defining $\mathbb{E}\left[\tilde{x}_{i j}(k)\right]=\chi_{i j}$, we have

$$
\begin{aligned}
\mathbb{E}\left[\tilde{x}_{j}^{(2)}(k)\right] & =\sum_{i=1}^{n} \chi_{i j}(k) \leq \mathbb{E}\left[q_{j}^{(2)}(k)\right]<\infty \forall j \in[m], \\
\mathbb{E}\left[\tilde{x}_{i}^{(1)}(k)\right] & =\sum_{j=1}^{m} \chi_{i j}(k) \leq \mathbb{E}\left[q_{i}^{(1)}(k)\right]<\infty \forall i \in[n], \\
\chi_{i j}(k) & =0 \forall(i, j) \notin E .
\end{aligned}
$$

Now substituting a and $\tilde{\mathbf{x}}$ in (28), we get (7b) (7c) and (7d $)$. Thus, for any pricing and matching policy under which the system is stable, the constraints in the fluid LP are necessary but not sufficient.

Proof of Proposition 2 Now, by ergodic theorem for Markov chains, the long run average reward converges to $\mathbb{E}[\mathcal{R}(\mathbf{q}, \mathbf{z})]$. Note that the Uniformized DTMC is aperiodic as we will always have transition from a state back to itself because of uniformization. Also, we have $\mathbb{E}[\mathcal{R}(\mathbf{q}, \mathbf{z})] \leq \mathbb{E}[\langle F(\boldsymbol{\lambda}), \boldsymbol{\lambda}\rangle-$ $\langle G(\boldsymbol{\mu}), \boldsymbol{\mu}\rangle] \leq\langle F(\tilde{\boldsymbol{\lambda}}), \tilde{\boldsymbol{\lambda}}\rangle-\langle G(\tilde{\boldsymbol{\mu}}), \tilde{\boldsymbol{\mu}}\rangle$ for any pricing and matching policy, where the last inequality follows from Jensen's Inequality and Assumption 2, Thus, the optimal fluid objective function value provides an upper bound for the average revenue under any stationary pricing and matching policy. Now, from the MDP theory, we know that there exists an optimal stationary policy and the fluid problem provides an upper bound for any stationary policy. Thus, fluid problem provides an upper bound for any arbitrary pricing and matching policy. Thus, the theorem follows. 


\section{Proof of Theorem 1}

We first define the following set for the ease of notation.

$$
\mathbb{C}^{E}=\left\{\boldsymbol{\lambda}, \boldsymbol{\mu}: \exists \boldsymbol{\chi} \in \mathbb{R}_{+}^{n \times m} \lambda_{j}=\sum_{i=1}^{n} \chi_{i j}, \forall j \in[m], \mu_{i}=\sum_{j=1}^{m} \chi_{i j}, \forall i \in[n], \chi_{i j}=0, \forall(i, j) \notin E\right\} .
$$

Note that these constraints are the same as the constraints in the fluid optimization problem (7b) (7c) (7d). We now present the proof of the theorem below.

Proof of Theorem 1] We will bound each of the terms in the profit-loss $\left(L^{\eta}\right)$ (9). In order to bound the first term, define a function of queue lengths $V(\mathbf{q})=\sum_{j=1}^{m}\left(q_{j}^{(2)}\right)^{2}$. Now, we will calculate $Q^{\eta} V(\mathbf{q})$ where $Q^{\eta}$ is the transition rate matrix of the $\eta^{t h}$ CTMC operating under the Fluid pricing policy and max-weight matching policy.

$$
\begin{aligned}
& Q^{\eta} V(\mathbf{q}) \\
= & \sum_{j=1}^{m} \eta \lambda_{j}^{*}\left[\sum_{j^{\prime} \in[m] / j}\left(q_{j^{\prime}}^{(2)}\right)^{2}+\left(q_{j}^{(2)}+\left(1-\sum_{i=1}^{m} y_{i j}^{(2)}\right) \mathbb{1}_{\left\{q_{j}^{(2)}<q_{\max }^{\eta}\right\}}\right)^{2}\right]+\sum_{i=1}^{m} \eta \mu_{i}^{*}\left[\sum_{j=1}^{m}\left(q_{j}^{(2)}-y_{i j}^{(1)}\right)^{2}\right] \\
& -\left[\sum_{i=1}^{m} \eta \mu_{i}^{*}+\sum_{j=1}^{m} \eta \lambda_{j}^{*}\right] \sum_{j=1}^{m}\left(q_{j}^{(2)}\right)^{2} \\
\stackrel{(a)}{=} & \sum_{j=1}^{m} \eta \lambda_{j}^{*}\left(1-\sum_{i=1}^{n} y_{i j}^{(2)}\right)\left(1+2 q_{j}^{(2)}\right) \mathbb{1}_{\left\{q_{j}^{(2)}<q_{\max }^{\eta}\right\}}+\sum_{i=1}^{n} \eta \mu_{i}^{*} \sum_{j=1}^{m} y_{i j}^{(1)}\left(1-2 q_{j}^{(2)}\right) \\
\stackrel{(b)}{\leq} & \sum_{i=1}^{n} \eta \mu_{i}^{*}+\sum_{j=1}^{m} \eta \lambda_{j}^{*}+2\left[\sum_{j=1}^{m} \eta \lambda_{j}^{*} q_{j}^{(2)}\left(1-\sum_{i=1}^{n} y_{i j}^{(2)}\right) \mathbb{1}_{\left\{q_{j}^{(2)}<q_{\max }^{\eta}\right\}}-\sum_{i=1}^{n} \eta \mu_{i}^{*} \sum_{j=1}^{m} q_{j}^{(2)} y_{i j}^{(1)}\right] \\
\stackrel{(c)}{=} & \sum_{i=1}^{n} \eta \mu_{i}^{*}+\sum_{j=1}^{m} \eta \lambda_{j}^{*}+2\left[\sum_{j=1}^{m} \eta \lambda_{j}^{*} q_{j}^{(2)} \mathbb{1}_{\left\{q_{j}^{(2)}<q_{\max }^{\eta}\right\}} \mathbb{1}_{\left\{\max _{i^{\prime}:\left(i^{\prime}, j\right) \in E} q_{i^{\prime}}^{(1)}=0\right\}}-\sum_{i=1}^{n} \eta \mu_{i}^{*}\left(\max _{j^{\prime}:\left(i, j^{\prime}\right) \in E} q_{j^{\prime}}^{(2)}\right)\right] \\
\stackrel{(d)}{=} & 2 \sum_{(i, j) \in E} \eta \chi_{i j}^{*}\left[1+q_{j}^{(2)} \mathbb{1}_{\left\{q_{j}^{(2)}<q_{\max }^{\eta}\right\}} \mathbb{1}_{\left\{\max _{i^{\prime}:\left(i^{\prime}, j\right) \in E} q_{i^{\prime}}^{(1)}=0\right\}}{\underset{j}{j^{\prime}:\left(i, j^{\prime}\right) \in E}}_{\left.\max _{j^{\prime}}\right]} q^{(2)}\right]
\end{aligned}
$$

where $(a)$ follows as $\left(y_{i j}^{(1)}\right)^{2}=y_{i j}^{(1)}$ as $y_{i j}^{(1)} \in\{0,1\}$. Also, $\left(1-\sum_{i=1}^{n} y_{i j}^{(2)}\right)^{2}=1-\sum_{i=1}^{n} y_{i j}^{(2)}$ as $\sum_{i=1}^{n} y_{i j}^{(2)} \in$ $\{0,1\}$. Next, (b) follows from the fact that $\left(1-\sum_{i=1}^{n} y_{i j}^{(2)}\right) \leq 1$ for all $j \in[m]$ and $\sum_{j=1}^{m} y_{i j}^{(1)} \leq 1$ for all $i \in[n]$ which is because there can be at most one arrival between two successive decision epochs and we only match the arriving customers and servers, i.e. we only match at most one pair in each time epoch. Now, $(c)$ follows from the max-weight matching algorithm 1 and $(d)$ follows as $\boldsymbol{\lambda}^{*}, \boldsymbol{\mu}^{*} \in \mathbb{C}$, there exists a $\boldsymbol{\chi}=\boldsymbol{\chi}^{*}$ such that $\boldsymbol{\lambda}^{*}, \boldsymbol{\mu}^{*}, \boldsymbol{\chi}^{*}$ satisfies (7b)-(7d). As $\mathbf{q} \leq \mathbf{1} q_{\max }^{\eta}, V(\mathbf{q})$ is finite for all permissible values of $\mathbf{q}$. Moreover, $\mathbb{E}[V(\mathbf{q})]$ is finite and so the family of CTMC for all $\eta$ are positive recurrent. Suppose $\mathbf{q}(\infty)$ denotes the steady state queue length vector. Taking expectation with respect to the steady state distribution of $\mathbf{q}$, we have $\mathbb{E}[Q V(\mathbf{q}(\infty))]=0$. So, we have

$$
0 \leq \sum_{(i, j) \in E} \chi_{i j}^{*}\left[1+\mathbb{E}\left[q_{j}^{(2)}(\infty) \mathbb{1}_{\left\{q_{j}^{(2)}(\infty)<q_{\max }^{\eta}\right\}} \mathbb{1}_{\left\{\max _{i^{\prime}:\left(i^{\prime}, j\right) \in E} q_{i^{\prime}}^{(1)}(\infty)=0\right\}}\right]-\mathbb{E}\left[\max _{j^{\prime}:\left(i, j^{\prime}\right) \in E} q_{j^{\prime}}^{(2)}(\infty)\right]\right]
$$




$$
\begin{aligned}
& \stackrel{*}{\leq} \sum_{(i, j) \in E} \chi_{i j}^{*}\left[1+\mathbb{E}\left[q_{j}^{(2)}(\infty)\right]-\mathbb{E}\left[I_{j}^{(2)}\left(q_{\max }^{\eta}\right)\right] q_{\max }^{\eta}-\mathbb{E}\left[\max _{j^{\prime}:\left(i, j^{\prime}\right) \in E} q_{j^{\prime}}^{(2)}(\infty)\right]\right] \\
& \stackrel{* *}{\leq} \sum_{(i, j) \in E} \chi_{i j}^{*}\left[1-\left[I_{j}^{(2)}\left(q_{\max }^{\eta}\right)\right] q_{\max }^{\eta}\right] \\
& =\sum_{j=1}^{m} \lambda_{j}^{*}-q_{\max }^{\eta} \sum_{j=1}^{m} \lambda_{j}^{*}\left[I_{j}^{(2)}\left(q_{\max }^{\eta}\right)\right] .
\end{aligned}
$$

where $(*)$ follows from the inequality $\mathbb{1}_{\max _{i^{\prime} \in N(j)} q_{i^{\prime}}^{(1)}(\infty)=0} \leq 1$ for all $\mathbf{q}(\infty)$ and $(* *)$ follows from the inequality $\max _{j^{\prime} \in N(i)} q_{j^{\prime}}^{(2)}(\infty) \geq q_{j}^{(2)}(\infty)$ for all $j$ such that $(i, j) \in E$. By substituting $q_{\max }^{\eta}=\gamma \sqrt{\eta}$ in (30) for an arbitrary positive constant $\gamma$, we get

$$
\begin{aligned}
\gamma \sqrt{\eta} \sum_{j=1}^{m} \lambda_{j}^{*} \mathbb{E}\left[I_{j}^{(2)}\left(q_{\max }^{\eta}\right)\right] & \leq \sum_{j=1}^{m} \lambda_{j}^{*}, \\
\Rightarrow \sum_{j=1}^{m} F_{j}\left(\lambda_{j}^{*}\right) \lambda_{j}^{*} \mathbb{E}\left[I_{j}^{(2)}\left(q_{\max }^{\eta}\right)\right] & \leq \frac{1}{\gamma \sqrt{\eta}} \max _{j \in[m]} F_{j}\left(\lambda_{j}^{*}\right) \sum_{j=1}^{m} \lambda_{j}^{*} .
\end{aligned}
$$

Thus, we have $\left\langle F\left(\boldsymbol{\lambda}^{*}\right), \boldsymbol{\lambda}^{*} \circ \mathbb{E}\left[\mathbf{I}^{(2)}\left(q_{\max }^{\eta}\right)\right]\right\rangle$ is $O(1 / \sqrt{\eta})$. Now we will bound the penalty term due to the expected queue length below:

The state space of the CTMC is such that for any state $\mathbf{q}$, we have $\mathbf{q} \leq q_{\max }^{\eta}$. Thus, it is trivially true that $\left\langle\mathbf{1}_{m+n}, \mathbb{E}[\mathbf{q}]\right\rangle \leq(m+n) q_{\max }^{\eta}=(m+n) \gamma \sqrt{\eta}$. Thus we can upper bound the profit loss $L^{\eta}$ by using (9) as follows:

$$
L^{\eta} \leq \sqrt{\eta}\left(\max _{j \in[m]} \frac{F_{j}\left(\lambda_{j}^{*}\right)}{\gamma} \sum_{j=1}^{m} \lambda_{j}^{*}+(m+n) \gamma\right)=O(\sqrt{\eta})
$$

\section{E. Proof of Proposition 3}

Proof of Proposition 3 In this proof, we omitted the subscript for the type of customer/server as we are working with a single link two sided queue. Under the fluid pricing policy, the steady state distribution of $q=q^{(2)}-q^{(1)}$ is uniform as it behaves like a symmetric simple random walk. Thus, the expected value of the sum of queue length $\left(q^{(1)}+q^{(2)}\right)$ can be computed in terms of the buffer capacity $q_{\max }^{\eta}$ as follows:

$$
\begin{aligned}
\mathbb{E}\left[q^{(1)}(k)+q^{(2)}(k)\right] & =\mathbb{E}\left[\left|q^{(1)}(k)-q^{(2)}(k)\right|\right] \\
& =\frac{q_{\max }^{\eta}\left(q_{\max }^{\eta}+1\right)}{2 q_{\max }^{\eta}+1} \\
& \approx q_{\max }^{\eta} / 2
\end{aligned}
$$

where $(*)$ is an approximation at large values of $q_{\max }^{\eta}$. We can also write the steady state probability of $q^{(1)}=q_{\max }^{\eta}$ and $q^{(2)}=q_{\max }^{\eta}$ by using the fact that the steady state queue length distribution is uniform across the state.

$$
\mathbb{P}\left[q^{(1)}=q_{\max }^{\eta}\right]=\frac{1}{2 q_{\max }^{\eta}+1}
$$




$$
\mathbb{P}\left[q^{(2)}=q_{\max }^{\eta}\right]=\frac{1}{2 q_{\max }^{\eta}+1}
$$

Now, we can evaluate the profit loss by using (9) as follows:

$$
L^{\eta}=\frac{F\left(\lambda^{*}\right) \lambda^{*}-G\left(\mu^{*}\right) \mu^{*}}{2 q_{\max }^{\eta}+1} \eta+s \frac{q_{\max }^{\eta}\left(q_{\max }^{\eta}+1\right)}{2 q_{\max }^{\eta}+1}
$$

Clearly, by (38), the asymptotic profit loss is optimal when the buffer is scaled as $q_{\max }^{\eta}=\gamma \sqrt{\eta}$ for some positive constant $\gamma$. To expound, if $q_{\max }^{\eta}=\gamma \eta^{0.5+\epsilon}$ for some $\epsilon>0$, then due to the second term, $L^{\eta}=\Theta\left(\eta^{0.5+\epsilon}\right)$. On the other hand if $\phi_{\max }^{\eta}=\gamma \eta^{0.5-\epsilon}$ for some $\epsilon>0$, then due to the first term, $L^{\eta}=\Theta\left(\eta^{0.5+\epsilon}\right)$.

As (38) holds with equality, the optimal profit loss is $\Theta(\sqrt{\eta})$. The proposition follows.

\section{F. Proof of Lemma 1}

We first present a definitions before proving the lemmas and theorem. We define the following set:

$$
\begin{gathered}
\mathbb{C}_{+}^{E}=\left\{\boldsymbol{\lambda}, \boldsymbol{\mu}: \exists \boldsymbol{\chi} \in \mathbb{R}_{+}^{n \times m}, \lambda_{j}=\sum_{i=1}^{n} \chi_{i j} \forall j \in[m], \mu_{i}=\sum_{j=1}^{m} \chi_{i j} \forall i \in[n],\right. \\
\left.\chi_{i j}=0 \forall(i, j) \notin E, \chi_{i j}>0 \forall(i, j) \in E\right\} .
\end{gathered}
$$

Unlike $\mathbb{C}^{E}$, in $\mathbb{C}_{+}^{E}$ we need $\chi_{i j}$ to be non zero for all $(i, j) \in E$. Note that, $\mathbb{C}_{+}^{E} \subseteq \mathbb{C}^{E}$, so if $\boldsymbol{\lambda}^{*}, \boldsymbol{\mu}^{*} \in \mathbb{C}_{+}^{E}$ then $\boldsymbol{\lambda}^{*}, \boldsymbol{\mu}^{*} \in \mathbb{C}^{E}$ but the converse may not be true. Also note that $\mathbb{C}^{E^{\prime}} \subseteq \mathbb{C}^{E}$ if $E^{\prime} \subseteq E$. Now we present the proof of the lemma below.

Proof of Lemma 1 We start by defining two Lyapunov Functions as,

$$
V^{(1)}(\mathbf{q})=\left\langle\mathbf{1}_{n},\left(\mathbf{q}^{(1)}\right)^{2}\right\rangle \text { and } V^{(2)}(\mathbf{q})=\left\langle\mathbf{1}_{m},\left(\mathbf{q}^{(2)}\right)^{2}\right\rangle
$$

Now, we will calculate the drift of $V^{(2)}($.$) for the \eta^{\text {th }}$ CTMC as follows:

$$
\begin{aligned}
& Q^{\eta} V^{(2)}(\mathbf{q}) \\
= & \sum_{j=1}^{m}\left(\eta \lambda_{j}^{*}-\theta_{j} \sigma^{\eta} \mathbb{1}_{\left\{q_{j}^{(2)}>\tau_{\max }^{\eta}\right\}}\right)\left(\left(q_{j}^{(2)}+1-\sum_{i=1}^{n} y_{i j}^{(2)}\right)^{2}-\left(q_{j}^{(2)}\right)^{2}\right) \\
& +\sum_{i=1}^{n}\left(\eta \mu_{i}^{*}-\phi_{i} \sigma^{\eta} \mathbb{1}_{\left\{q_{i}^{(1)}>\tau_{\max }^{\eta}\right\}}\right) \sum_{j=1}^{m}\left(\left(q_{j}^{(2)}-y_{i j}^{(1)}\right)^{2}-\left(q_{j}^{(2)}\right)^{2}\right) \\
\stackrel{(a)}{=} & \sum_{j=1}^{m}\left(\eta \lambda_{j}^{*}-\theta_{j} \sigma^{\eta} \mathbb{1}_{\left\{q_{j}^{(2)}>\tau_{\max }^{\eta}\right\}}\right)\left(1-\sum_{i=1}^{n} y_{i j}^{(2)}\right)\left(1+2 q_{j}^{(2)}\right) \\
& +\sum_{i=1}^{n}\left(\eta \mu_{i}^{*}-\phi_{i} \sigma^{\eta} \mathbb{1}_{\left\{q_{i}^{(1)}>\tau_{\max }^{\eta}\right\}}\right) \sum_{j=1}^{m} y_{i j}^{(1)}\left(1-2 q_{j}^{(2)}\right) \\
\stackrel{(b)}{\leq} & \eta\left\langle\mathbf{1}_{m}, \boldsymbol{\lambda}^{*}\right\rangle+\eta\left\langle\mathbf{1}_{n}, \boldsymbol{\mu}^{*}\right\rangle+2 \sum_{j=1}^{m} \eta \lambda_{j}^{*}\left(1-\sum_{i=1}^{n} y_{i j}^{(2)}\right) q_{j}^{(2)}-2 \sum_{i=1}^{n} \eta \mu_{i}^{*} \sum_{j=1}^{m} y_{i j}^{(1)} q_{j}^{(2)}
\end{aligned}
$$




$$
\begin{aligned}
& -2 \sigma^{\eta} \sum_{j=1}^{m} \theta_{j}\left(1-\sum_{i=1}^{n} y_{i j}^{(2)}\right) q_{j}^{(2)} \mathbb{1}_{\left\{q_{j}^{(2)}>\tau_{\max }^{\eta}\right\}}+2 \sigma^{\eta} \sum_{i=1}^{n} \phi_{i} \sum_{j=1}^{m} y_{i j}^{(1)} q_{j}^{(2)} \mathbb{1}_{\left\{q_{i}^{(1)}>\tau_{\max }^{\eta}\right\}} \\
& \stackrel{(c)}{=} \eta\left\langle\mathbf{1}_{m}, \boldsymbol{\lambda}^{*}\right\rangle+\eta\left\langle\mathbf{1}_{n}, \boldsymbol{\mu}^{*}\right\rangle+2 \sum_{j=1}^{m} \eta \lambda_{j}^{*} q_{j}^{(2)} \mathbb{1}_{\left\{\max _{i^{\prime}:\left(i^{\prime}, j\right) \in E^{*}} q_{i^{\prime}}^{(1)}=0\right\}}-2 \sum_{i=1}^{n} \eta \mu_{i}^{*} \max _{j^{\prime}:\left(i, j^{\prime}\right) \in E^{*}} q_{j^{\prime}}^{(2)} \\
& \left.\quad-2 \sigma^{\eta} \sum_{j=1}^{m} \theta_{j} q_{j}^{(2)} \mathbb{1}_{\left\{q_{j}^{(2)}>\tau_{\max }^{\eta}\right\}} \mathbb{1}_{\left\{\max _{i^{\prime}:\left(i^{\prime}, j\right) \in E^{*}} q_{i^{\prime}}^{(1)}=0\right\}}+2 \sigma^{\eta} \sum_{i=1}^{n} \phi_{i} \max _{j^{\prime}:\left(i, j^{\prime}\right) \in E^{*}} q_{j^{\prime}}^{(2)} \mathbb{1}_{\left\{q_{i}^{(1)}>\tau_{\max }^{\eta}\right\}}\right\} \\
& \stackrel{(d)}{=} \eta\left\langle\mathbf{1}_{m}, \boldsymbol{\lambda}^{*}\right\rangle+\eta\left\langle\mathbf{1}_{n}, \boldsymbol{\mu}^{*}\right\rangle+2 \eta \sum_{i, j \in E^{*}} \chi_{i j}^{*}\left(q_{j}^{(2)} \mathbb{1}_{\left\{\max _{i^{\prime}:\left(i^{\prime}, j\right) \in E^{*}} q_{i^{\prime}}^{(1)}=0\right\}}-\max _{j^{\prime}:\left(i, j^{\prime}\right) \in E^{*}} q_{j^{\prime}}^{(2)}\right) \\
& \quad-2 \sigma^{\eta} \sum_{j=1}^{m} \theta_{j} q_{j}^{(2)} \mathbb{1}_{\left\{q_{j}^{(2)}>\tau_{\max }^{\eta}\right\}} \\
& \stackrel{(e)}{\leq} \eta\left\langle\mathbf{1}_{m}, \boldsymbol{\lambda}^{*}\right\rangle+\eta\left\langle\mathbf{1}_{n}, \boldsymbol{\mu}^{*}\right\rangle-2 \sigma^{\eta} \sum_{j=1}^{m} \theta_{j} q_{j}^{(2)} \mathbb{1}_{\left\{q_{j}^{(2)}>\tau_{\max }^{\eta}\right\}} .
\end{aligned}
$$

Under the modified max-weight matching policy, if any of the compatible counterparts' queue (according to $E^{*}$ ) of the arrival is non empty, we match it with the type with most number of waiting customers/ servers. Thus, we will have $\sum_{i=1}^{n} y_{i j}^{(2)}$ and $y_{i j}^{(1)}$ for all $j$ to be either 1 or 0 . Thus, we will have $\left(1-\sum_{i=1}^{n} y_{i j}^{(2)}\right)^{2}=1-\sum_{i=1}^{n} y_{i j}^{(2)}$ and $\left(y_{i j}^{(1)}\right)^{2}=y_{i j}^{(1)}$. Thus, $((a))$ follows.

We have $\eta \lambda_{j}^{*}-\theta_{j} \sigma^{\eta} \mathbb{1}_{\left\{q_{j}^{(2)}>\tau_{\max }^{\eta}\right\}}<\eta \lambda_{j}^{*}$ as $\theta_{j} \sigma^{\eta}>0$ and $1-\sum_{i=1}^{n} y_{i j}^{(2)} \leq 1$. We also have that $\eta \mu_{i}^{*}-\phi_{i} \sigma^{\eta} \mathbb{1}_{\left\{q_{i}^{(1)}>\tau_{\max }^{\eta}\right\}}<\eta \mu_{i}^{*}$ as $\phi_{i} \sigma^{\eta}>0$ and $\sum_{j=1}^{m} y_{i j}^{(1)} \leq 1$. Using these inequalities, $((b))$ follows.

Now, $((c))$ follows from the Algorithm 1, as we match whenever any of the compatible counterparts' queue in the minimal capacity serving graph is empty and we match it to the queue with maximum number of customers/servers waiting.

We can have $q_{i}^{(1)}>\tau_{\max }^{\eta}>0$, if and only if the queues of all the compatible counterparts of $i$ in minimal capacity serving graphs are empty as we match immediately whenever there is an arrival. Thus, the last term in (39) is 0 and thus, $((d))$ follows. We also use the definition of the capacity region and the fact that $\boldsymbol{\lambda}^{*}, \boldsymbol{\mu}^{*} \in \mathbb{C}_{+}^{E^{*}}$ in this step.

Lastly, $(e)$ follows as the last term is zero when $\chi_{i j}^{*}=0$ and we have $j:(i, j) \in E^{*}$ when $\chi_{i j}^{*}>0$ and thus, $q_{j}^{(2)} \leq \max _{j^{\prime}:\left(i, j^{\prime}\right) \in E^{*}} q_{j^{\prime}}^{(2)}$. Now, we can similarly upper bound the drift of $V^{(1)}(\mathbf{q})$. We have

$$
Q^{\eta} V^{(1)}(\mathbf{q}) \leq \eta\left\langle\mathbf{1}_{m}, \boldsymbol{\lambda}^{*}\right\rangle+\eta\left\langle\mathbf{1}_{n}, \boldsymbol{\mu}^{*}\right\rangle-2 \sigma^{\eta} \sum_{i=1}^{n} \phi_{i} q_{i}^{(1)} \mathbb{1}_{\left\{q_{i}^{(1)}>\tau_{\max }^{\eta}\right\}} .
$$

Now, if we add (40) and (41), we will have an upper bound on the drift of the Lyapunov function $V(\mathbf{q})=V^{(1)}(\mathbf{q})+V^{(2)}(\mathbf{q})$ to be:

$$
Q^{\eta} V(\mathbf{q}) \leq B-2 \sigma^{\eta} \sum_{i=1}^{n} \phi_{i} q_{i}^{(1)} \mathbb{1}_{\left\{q_{i}^{(1)}>\tau_{\max }^{\eta}\right\}}-2 \sigma^{\eta} \sum_{j=1}^{m} \theta_{j} q_{j}^{(2)} \mathbb{1}_{\left\{q_{j}^{(2)}>\tau_{\max }^{\eta}\right\}},
$$


Where, $B=2 \eta\left\langle\mathbf{1}_{m}, \boldsymbol{\lambda}^{*}\right\rangle+2 \eta\left\langle\mathbf{1}_{n}, \boldsymbol{\mu}^{*}\right\rangle$. Now consider the following set:

$$
\mathcal{B}^{\eta}=\left\{\mathbf{q}: q_{i}^{(1)} \leq \max \left\{\frac{B}{\phi_{i} \sigma^{\eta}}, \tau_{\max }^{\eta}\right\}, q_{j}^{(2)} \leq \max \left\{\frac{B}{\theta_{j} \sigma^{\eta}}, \tau_{\max }^{\eta}\right\} \forall i \in[n], j \in[m]\right\} .
$$

Outside the finite set $\mathcal{B}^{\eta}$, the drift of the Lyapunov function $V(\mathbf{q})$ is strictly less than zero. We have

$$
Q^{\eta} V^{(1)}(\mathbf{q})+Q^{\eta} V^{(2)}(\mathbf{q}) \leq-B<0 \forall \mathbf{q} \in\left(\mathcal{B}^{\eta}\right)^{c}
$$

Thus, the system is positive recurrent for any $\eta$ and the first part of the lemma follows. Now, we will use the moment bound theorem to upper bound the expectation of the sum of the queue lengths to get the desired inequality. We have,

$$
\sigma^{\eta} \mathbb{E}\left[\sum_{i=1}^{n} \phi_{i} q_{i}^{(1)} \mathbb{1}_{\left\{q_{i}^{(1)}>\tau_{\max }^{\eta}\right\}}+\sum_{j=1}^{m} \theta_{j} q_{j}^{(2)} \mathbb{1}_{\left\{q_{j}^{(2)}>\tau_{\max }^{\eta}\right\}}\right] \leq \frac{B}{2}
$$

By substituting $\mathbb{1}_{\left\{q_{i}^{(1)}>\tau_{\max }^{\eta}\right\}}=1-\mathbb{1}_{\left\{q_{i}^{(1)} \leq \tau_{\max }^{\eta}\right\}}$ and then bounding the RHS by using the inequality $\mathbb{E}\left[q_{i}^{(1)} \mathbb{1}_{\left\{q_{i}^{(1)} \leq \tau_{\max }^{\eta}\right\}}\right] \leq \tau_{\max }^{\eta}$, we get the lemma.

\section{G. Proof of Lemma 2}

Before proving the lemma, we first prove the following claim:

Claim 2. Consider a pricing and matching policy and assume the following:

- The multiple link two sided queue is positive recurrent

- $\mathbb{E}\left[\left\langle\mathbf{1}_{m+n}, \mathbf{q}\right\rangle\right]<\infty$ under the given policy

- The pricing policy is of the following form:

$$
\begin{aligned}
& \tilde{\lambda}_{j}=\eta \lambda_{j}^{*}+\tilde{f}_{j}(\mathbf{q}, \eta) \forall j \in[m] \\
& \tilde{\mu}_{i}=\eta \mu_{i}^{*}+\tilde{g}_{i}(\mathbf{q}, \eta) \forall i \in[n],
\end{aligned}
$$

Then we have

$$
\sum_{j \in[m]}\left(F_{j}^{\prime}\left(\lambda_{j}^{*}\right) \lambda_{j}^{*}+F_{j}\left(\lambda_{j}^{*}\right)\right) \mathbb{E}\left[\tilde{f}_{j}(\mathbf{q}, \eta)\right] \leq \sum_{i \in[n]}\left(G_{i}^{\prime}\left(\mu_{i}^{*}\right) \mu_{i}^{*}+G_{i}\left(\mu_{i}^{*}\right)\right) \mathbb{E}\left[\tilde{g}_{i}(\mathbf{q}, \eta)\right]
$$

If in addition, the matching policy only uses edges $E^{*}$ from the minimal capacity serving graph to match, then we have

$$
\sum_{j \in[m]}\left(F_{j}^{\prime}\left(\lambda_{j}^{*}\right) \lambda_{j}^{*}+F_{j}\left(\lambda_{j}^{*}\right)\right) \mathbb{E}\left[\tilde{f}_{j}(\mathbf{q}, \eta)\right]=\sum_{i \in[n]}\left(G_{i}^{\prime}\left(\mu_{i}^{*}\right) \mu_{i}^{*}+G_{i}\left(\mu_{i}^{*}\right)\right) \mathbb{E}\left[\tilde{g}_{i}(\mathbf{q}, \eta)\right]
$$


Proof of the Claim First, we will define a vector $\bar{\chi}$ of dimension $\mathbb{R}^{(m n) \times 1}$ given a matrix $\chi$ of dimension $\mathbb{R}^{n \times m}$ as follows:

$$
\bar{\chi}_{k}=\chi_{i j} \text { where } i=\left\lceil\frac{k}{m}\right\rceil j=k \%(m+1) \forall k \in[m n],
$$

where $\lceil x\rceil$ is the smallest integer greater than or equal to $x$ and $x \%(n+1)$ is the reminder obtained by dividing $x \in \mathbb{Z}_{+}$by $(n+1)$. In other words, $\bar{\chi}$ is a vector obtained by stacking all the rows of the $\chi$ matrix next to each other.

By the hypothesis of the claim, the system is positive recurrent. Also, by the Claim 1, we know that the constraints (7b) (7c) (7d ) are necessarily satisfied for the pricing and matching policy under which the system is positive recurrent and $\mathbb{E}\left[\left\langle\mathbf{1}_{m+n}, \mathbf{q}\right\rangle\right]<\infty$. Thus, for the arrival rate vector $(\tilde{\boldsymbol{\lambda}}, \tilde{\boldsymbol{\mu}})$, there exists a corresponding average rate assignment vector $\overline{\boldsymbol{\chi}}^{p}$ corresponding to the rate assignment matrix $\chi^{p}$ such that the constraints which define the set $\mathbb{C}^{E}$, which is the same as the constraints (7b) (7c) (7d $)$ is satisfied.

Now we restate the fluid problem originally defined in (7ad (77c) (7b) (7d ) below:

$$
\max f(\boldsymbol{\lambda}, \boldsymbol{\mu}, \bar{\chi})=\langle F(\boldsymbol{\lambda}), \boldsymbol{\lambda}\rangle-\langle G(\boldsymbol{\mu}), \boldsymbol{\mu}\rangle
$$

subject to,

$$
\begin{aligned}
& h_{j}^{(2)}(\boldsymbol{\lambda}, \boldsymbol{\mu}, \bar{\chi})=\lambda_{j}-\sum_{k: k \%(m+1)=j} \bar{\chi}_{k}=0 \forall j \in[m], \\
& h_{i}^{(1)}(\boldsymbol{\lambda}, \boldsymbol{\mu}, \overline{\boldsymbol{\chi}})=\mu_{i}-\sum_{k:\lceil k / m\rceil=i} \bar{\chi}_{k}=0 \forall i \in[n], \\
& \bar{\chi}_{k}=0 \forall(\lceil k / m\rceil, k \%(m+1)) \notin E, \bar{\chi}_{k} \geq 0 \forall(\lceil k / m\rceil, k \%(m+1)) \in E .
\end{aligned}
$$

As $(\tilde{\boldsymbol{\lambda}}, \tilde{\boldsymbol{\mu}}) \in \mathbb{C}^{E}$, there exists a corresponding average rate assignment vector $\overline{\boldsymbol{\chi}}^{p} \in \mathbb{R}_{+}^{n \times m}$ corresponding to the average rate assignment matrix $\chi^{p}$, that comes from the given pricing policy (42), (43) and matching policy such that $\chi_{i j}^{p}=0 \forall(i, j) \notin E$ and $\mathbf{h}\left(\tilde{\boldsymbol{\lambda}}, \tilde{\boldsymbol{\mu}}, \boldsymbol{\chi}^{p}\right)=\mathbf{0}_{m+n}$. Here we define $\mathbf{h}: \mathbb{R}^{m+n+m n} \rightarrow \mathbb{R}^{m+n}$ as

$$
\mathbf{h}(.)=\left(h_{1}^{(2)}(.), h_{2}^{(2)}(.), \ldots h_{m}^{(2)}, h_{1}^{(1)}(.), \ldots h_{n}^{(1)}(.)\right)
$$

which is the concatenation all the equality constraints.

To prove the claim, we will use the optimality condition for the fluid problem (7a) (7c) (7b ) (7d) as $\left(\boldsymbol{\lambda}^{*}, \boldsymbol{\mu}^{*}, \boldsymbol{\chi}^{*}\right)$ is an optimal solution to this problem. We will do the following steps:

- First we will argue that the optimal point $\left(\boldsymbol{\lambda}^{*}, \boldsymbol{\mu}^{*}, \boldsymbol{\chi}^{*}\right)$ is regular, i.e. the constraints tight at the optimal point are linearly independent.

- Then we will use the arrival rates of the given price policy to find a feasible direction for the fluid problem 
- Finally, we will use the first order KKT optimality conditions

The gradients of the active constraints at the optimal point of the fluid problem $\left(\boldsymbol{\lambda}^{*}, \boldsymbol{\mu}^{*}, \boldsymbol{\chi}^{*}\right)$ are linearly independent as the constraints $\mathbf{h}(\boldsymbol{\lambda}, \boldsymbol{\mu}, \boldsymbol{\chi})$ and the non negativity constraints are linear and it can be easily verified that the normal vectors to the hyper planes and half spaces corresponding to these constraints are linearly independent.

So, the optimal point is regular and thus, there exists unique Lagrangian multipliers $\left(\boldsymbol{\kappa}^{*}, \boldsymbol{\xi}^{*}\right) \in$ $\mathbb{R}^{m+n} \times \mathbb{R}_{+}^{m \times n}$ such that,

$$
\nabla f\left(\boldsymbol{\lambda}^{*}, \boldsymbol{\mu}^{*}, \bar{\chi}^{*}\right)+\nabla \mathbf{h}\left(\boldsymbol{\lambda}^{*}, \boldsymbol{\mu}^{*}, \overline{\boldsymbol{\chi}}^{*}\right) \boldsymbol{\kappa}^{*}+\sum_{k:(\lceil k / m\rceil, k \%(m+1)) \in E} \xi_{k}^{*} \mathbf{e}_{k+n+m} \mathbb{1}_{\bar{\chi}_{k}^{*}=0}+\sum_{k:(\lceil k / m\rceil, k \%(m+1)) \notin E} \xi_{k}^{*} \mathbf{e}_{k+n+m}=\mathbf{0}_{m+n+m n},
$$

where $f\left(\boldsymbol{\lambda}^{*}, \boldsymbol{\mu}^{*}, \boldsymbol{\chi}^{*}\right) \in \mathbb{R}^{m+n+m n}$ is given by

$$
\nabla f\left(\boldsymbol{\lambda}^{*}, \boldsymbol{\mu}^{*}, \boldsymbol{\chi}^{*}\right)=\left(F^{\prime}\left(\boldsymbol{\lambda}^{*}\right) \boldsymbol{\lambda}^{*}+F\left(\boldsymbol{\lambda}^{*}\right),-G^{\prime}\left(\boldsymbol{\mu}^{*}\right) \boldsymbol{\mu}^{*}-G\left(\boldsymbol{\mu}^{*}\right), \mathbf{0}_{n m}\right) .
$$

Also, as $\mathbf{h}: \mathbb{R}^{m+n+m n} \rightarrow \mathbb{R}^{m+n}$, the gradient $\nabla h\left(\boldsymbol{\lambda}^{*}, \boldsymbol{\mu}^{*}, \bar{\chi}^{*}\right)$ is a matrix in $\mathbb{R}^{(m+n+m n) \times(m+n)}$ where each column corresponds to the the gradient of the equality constraints $h_{j}^{(2)}$ for all $j \in[m]$ and $h_{i}^{(1)}$ for all $i \in[n]$. Finally, we define $\mathbf{e}_{k+n+m} \in \mathbb{R}^{m+n+m n}$ as a vector with all components zero except the $(k+n+m)^{t h}$ component. Now we define a vector $\mathbf{d} \in \mathbb{R}^{n+m+m n}$ which is a feasible direction for the optimization problem, given by

$$
\begin{aligned}
\mathbf{d} & =\left(\boldsymbol{\lambda}^{*}, \boldsymbol{\mu}^{*}, \overline{\boldsymbol{\chi}}^{*}\right)-\left(\tilde{\boldsymbol{\lambda}}, \tilde{\boldsymbol{\mu}}, \overline{\boldsymbol{\chi}}^{p}\right) \\
\Rightarrow d_{k} & =\left\{\begin{array}{l}
\mathbb{E}\left[\tilde{f}_{j}(\mathbf{q}, \eta)\right], \forall k \in[m] \\
\mathbb{E}\left[\tilde{g}_{i}(\mathbf{q}, \eta)\right], \forall j \in m+[n] \\
\bar{\chi}_{k}^{*}-\bar{\chi}_{k}^{p}, \forall k \in m+n+[m n] .
\end{array}\right.
\end{aligned}
$$

Now we have,

$$
\begin{gathered}
\left\langle\mathbf{d}, \nabla h_{j}^{(2)}\right\rangle=h_{j}^{(2)}\left(\boldsymbol{\lambda}^{*}, \boldsymbol{\mu}^{*}, \boldsymbol{\chi}^{*}\right)-h_{j}^{(2)}\left(\tilde{\boldsymbol{\lambda}}, \tilde{\boldsymbol{\mu}}, \overline{\boldsymbol{\chi}}^{p}\right)=0, \forall j \in[m] \\
\left\langle\mathbf{d}, \nabla h_{i}^{(1)}\right\rangle=h_{i}^{(1)}\left(\boldsymbol{\lambda}^{*}, \boldsymbol{\mu}^{*}, \boldsymbol{\chi}^{*}\right)-h_{i}^{(1)}\left(\mathbb{E}\left[\tilde{\boldsymbol{\lambda}}, \tilde{\boldsymbol{\mu}}, \overline{\boldsymbol{\chi}}^{p}\right)=0, \forall i \in[n]\right. \\
\sum_{k:(\lceil k / m\rceil, k \%(m+1)) \in E} \bar{\xi}_{k}\left(\bar{\chi}_{k}^{*}-\bar{\chi}_{k}^{p}\right) \mathbb{1}_{\bar{\chi}_{k}^{*}=0}+\sum_{k:(\lceil k / m\rceil, k \%(m+1)) \notin E} \xi_{k}\left(\bar{\chi}_{k}^{*}-\bar{\chi}_{k}^{p}\right) \stackrel{*}{\leq} 0,
\end{gathered}
$$

where $(*)$ follows from the from the fact that $\chi_{i j}^{p}=0$ and $\chi_{i j}^{*}=0$ for all $(i, j) \notin E$ Thus, by taking the inner product on both the side by $\mathbf{d}$ in (44) and using (45), (46) and (47) we get $\left\langle\mathbf{d}, \nabla f\left(\boldsymbol{\lambda}^{*}, \boldsymbol{\mu}^{*}, \bar{\chi}^{*}\right)\right\rangle \leq 0$. Now, by expanding $\left\langle\mathbf{d}, \nabla f\left(\boldsymbol{\lambda}^{*}, \boldsymbol{\mu}^{*}, \bar{\chi}^{*}\right)\right\rangle$, we have the first part of the claim.

If in addition, if we use edges only from $E^{*}$, then we will have $\chi_{i j}^{p}=0$ and $\chi_{i j}^{*}=0$ for all $(i, j) \notin E^{*}$ and $\chi_{i j}^{*}>0$ for all $(i, j) \in E^{*}$. As $E^{*} \subseteq E$ by definition, we have

$$
\sum_{k:(\lceil k / m\rceil, k \%(m+1)) \in E} \bar{\xi}_{k}\left(\bar{\chi}_{k}^{*}-\bar{\chi}_{k}^{p}\right) \mathbb{1}_{\bar{\chi}_{k}^{*}=0}+\sum_{k:(\lceil k / m\rceil, k \%(m+1)) \notin E} \xi_{k}\left(\bar{\chi}_{k}^{*}-\bar{\chi}_{k}^{p}\right)=0
$$


Thus, we will have $\left\langle\mathbf{d}, \nabla f\left(\boldsymbol{\lambda}^{*}, \boldsymbol{\mu}^{*}, \bar{\chi}^{*}\right)\right\rangle=0$. Now, by equating $\left\langle\mathbf{d}, \nabla f\left(\boldsymbol{\lambda}^{*}, \boldsymbol{\mu}^{*}, \bar{\chi}^{*}\right)\right\rangle$ to zero, we have the claim.

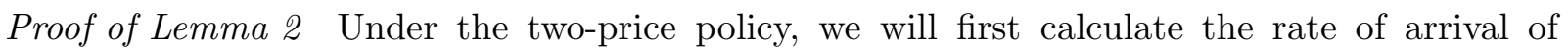
requests. We have

$$
\begin{aligned}
\mathbb{E}\left[a_{j}^{(2)}\right] & =\mathbb{E}\left[a_{j}^{(2)} \mid q_{j}^{(2)} \leq \tau_{\max }^{\eta}\right] \mathbb{P}\left[q_{j}^{(2)} \leq \tau_{\max }^{\eta}\right]+\mathbb{E}\left[a_{j}^{(2)} \mid q_{j}^{(2)}>\tau_{\max }^{\eta}\right] \mathbb{P}\left[q_{j}^{(2)}>\tau_{\max }^{\eta}\right] \\
& =\lambda_{j}^{*}-\theta_{j} \sigma^{\eta} \mathbb{P}\left[q_{j}^{(2)}>\tau_{\max }^{\eta}\right] \forall j \in[m],
\end{aligned}
$$

Similarly, we also have:

$$
\mathbb{E}\left[a_{i}^{(1)}\right]=\mu_{i}^{*}-\phi_{i} \sigma^{\eta} \mathbb{P}\left[q_{i}^{(1)}>\tau_{\max }^{\eta}\right] \forall i \in[n]
$$

Now we define a vector $\mathbf{d} \in \mathbb{R}^{n+m+m n}$ which is a feasible direction for the optimization problem, given by

$$
\begin{aligned}
\mathbf{d} & =\left(\boldsymbol{\lambda}^{*}, \boldsymbol{\mu}^{*}, \overline{\boldsymbol{\chi}}^{*}\right)-\left(\mathbb{E}[\mathbf{a}], \bar{\chi}^{T P}\right) \\
\Rightarrow d_{k} & =\left\{\begin{array}{l}
\theta_{k} \sigma^{\eta} \mathbb{P}\left[q_{k}^{(2)}>\tau_{\max }^{\eta}\right], \forall k \in[m] \\
\phi_{k} \sigma^{\eta} \mathbb{P}\left[q_{k}^{(1)}>\tau_{\max }^{\eta}\right], \forall j \in m+[n] \\
\bar{\chi}_{k}^{*}-\bar{\chi}_{k}^{T P}, \forall k \in m+n+[m n] .
\end{array}\right.
\end{aligned}
$$

where $\bar{\chi}^{T P} \in \mathbb{R}_{+}^{m n}$ is the rate assignment vector for the two-price policy, analogous to $\bar{\chi}^{p}$ for a general pricing policy. By Lemma 1, the system is positive recurrent under the two-price policy for any $\boldsymbol{\theta}^{\eta}>\mathbf{0}_{m}, \boldsymbol{\phi}^{\eta}>\mathbf{0}_{n}$ and $\sigma^{\eta}$ with $\mathbb{E}\left[\left\langle\mathbf{1}_{m+n}, \mathbf{q}\right\rangle\right]<\infty$. Next, as we are using modified max weight policy, we only match using edges in $E^{*}$. Finally, the two-price policy falls under the form given in (42) and (43). Thus, by Claim 2, we have $\left\langle\mathbf{d}, \nabla f\left(\boldsymbol{\lambda}^{*}, \boldsymbol{\mu}^{*}, \bar{\chi}^{*}\right)\right\rangle=0$. Thus, the lemma follows.

\section{H. Proof of Theorem 2}

Proof of Theorem 2 We will first calculate the profit loss given by (9) as follows:

$$
\begin{aligned}
& L^{\eta} \\
= & \pi_{*}^{\eta}-\left(\pi^{\eta}-s\left\langle\mathbf{1}_{n+m}, \mathbb{E}[\mathbf{q}]\right\rangle\right) \\
= & \eta\left\langle F\left(\boldsymbol{\lambda}^{*}\right), \boldsymbol{\lambda}^{*}\right\rangle-\eta\left\langle G\left(\boldsymbol{\mu}^{*}\right), \boldsymbol{\mu}^{*}\right\rangle-\eta\left(\sum_{j \in[m]} F_{j}\left(\lambda_{j}^{*}\right) \lambda_{j}^{*} \mathbb{P}\left[q_{j}^{(2)} \leq \tau_{\max }^{\eta}\right]+\right. \\
& \sum_{j \in[m]} F_{j}\left(\lambda_{j}^{*}-\frac{\theta_{j} \sigma^{\eta}}{\eta}\right)\left(\lambda_{j}^{*}-\frac{\theta_{j} \sigma^{\eta}}{\eta}\right) \mathbb{P}\left[q_{j}^{(2)}>\tau_{\max }^{\eta}\right]-\sum_{i \in[n]} G_{i}\left(\mu_{i}^{*}\right) \mu_{i}^{*} \mathbb{P}\left[q_{i}^{(1)} \leq \tau_{\max }^{\eta}\right] \\
& \left.-\sum_{i \in[n]} G_{i}\left(\mu_{i}^{*}-\frac{\phi_{i} \sigma^{\eta}}{\eta}\right)\left(\mu_{i}^{*}-\frac{\phi_{i} \sigma^{\eta}}{\eta}\right) \mathbb{P}\left[q_{i}^{(1)}>\tau_{\max }^{\eta}\right]\right)+s\left\langle\mathbf{1}_{m+n}, \mathbb{E}[\mathbf{q}]\right\rangle . \\
= & \eta \sum_{j \in[m]}\left(F_{j}\left(\lambda_{j}^{*}\right) \lambda_{j}^{*}-F_{j}\left(\lambda_{j}^{*}-\frac{\theta_{j} \sigma^{\eta}}{\eta}\right)\left(\lambda_{j}^{*}-\frac{\theta_{j} \sigma^{\eta}}{\eta}\right)\right) \mathbb{P}\left[q_{j}^{(2)}>\tau_{\max }^{\eta}\right]
\end{aligned}
$$




$$
\begin{aligned}
& -\eta \sum_{i \in[n]}\left(G_{i}\left(\mu_{i}^{*}\right) \mu_{i}^{*}-G_{i}\left(\mu_{i}^{*}-\frac{\phi_{i} \sigma^{\eta}}{\eta}\right)\left(\mu_{i}^{*}-\frac{\phi_{i} \sigma^{\eta}}{\eta}\right)\right) \mathbb{P}\left[q_{i}^{(1)}>\tau_{\max }^{\eta}\right]+s\left\langle\mathbf{1}_{m+n}, \mathbb{E}[\mathbf{q}]\right\rangle . \\
\stackrel{*}{=} & \sum_{j \in[m]}\left(F_{j}^{\prime}\left(\lambda_{j}^{*}\right) \lambda_{j}^{*}+F_{j}\left(\lambda_{j}^{*}\right)\right) \theta_{j} \sigma^{\eta} \mathbb{P}\left[q_{j}^{(2)}>\tau_{\max }^{\eta}\right]+s\left\langle\mathbf{1}_{m+n}, \mathbb{E}[\mathbf{q}]\right\rangle- \\
& \sum_{i \in[n]}\left(G_{i}^{\prime}\left(\mu_{i}^{*}\right) \mu_{i}^{*}+G_{i}\left(\mu_{i}^{*}\right)\right) \phi_{i} \sigma^{\eta} \mathbb{P}\left[q_{i}^{(1)}>\tau_{\max }^{\eta}\right]+O\left(\eta^{1 / 3}\right),
\end{aligned}
$$

where $(*)$ follows from the Taylor series expansion of the terms $F\left(\boldsymbol{\lambda}^{*}-\boldsymbol{\theta} \sigma^{\eta} / \eta\right)$ and $G\left(\boldsymbol{\mu}^{*}-\boldsymbol{\phi} \sigma^{\eta} / \eta\right)$ component wise and using $\sigma^{\eta}=\eta^{2 / 3}$. To expound, for type $j$ customer, we can obtain $(*)$ by the following steps:

$$
\begin{aligned}
& \eta F_{j}\left(\lambda_{j}^{*}\right) \lambda_{j}^{*}-\eta F_{j}\left(\lambda_{j}^{*}-\frac{\theta_{j} \sigma^{\eta}}{\eta}\right)\left(\lambda_{j}^{*}-\frac{\theta_{j} \sigma^{\eta}}{\eta}\right) \\
& =\eta F_{j}\left(\lambda_{j}^{*}\right) \lambda_{j}^{*}-\eta\left(F_{j}\left(\lambda_{j}^{*}\right)-\frac{\theta_{j} \sigma^{\eta}}{\eta} F_{j}^{\prime}\left(\lambda_{j}^{*}\right)+\left(\frac{\theta_{j} \sigma^{\eta}}{\eta}\right)^{2} F_{j}^{\prime \prime}\left(\lambda_{j}^{*}\right)+O\left(\eta^{-2 / 3}\right)\right)\left(\lambda_{j}^{*}-\frac{\theta_{j} \sigma^{\eta}}{\eta}\right) \\
& =\left(F_{j}\left(\lambda_{j}^{*}\right)+F_{j}^{\prime}\left(\lambda_{j}^{*}\right) \lambda_{j}^{*}\right) \theta_{j} \sigma^{\eta}-\left(F_{j}^{\prime \prime}\left(\lambda_{j}^{*}\right) \lambda_{j}^{*}+F_{j}^{\prime}\left(\lambda_{j}^{*}\right)\right) \frac{\left(\theta \sigma^{\eta}\right)^{2}}{\eta}+O\left(\eta^{1 / 3}\right) \\
& =\left(F_{j}\left(\lambda_{j}^{*}\right)+F_{j}^{\prime}\left(\lambda_{j}^{*}\right) \lambda_{j}^{*}\right) \theta_{j} \sigma^{\eta}+O\left(\eta^{1 / 3}\right) .
\end{aligned}
$$

By using Lemma 2, we can simplify the profit loss for the $\eta^{\text {th }}$ system by eliminating the first order terms to get:

$$
L^{\eta} \leq O\left(\eta^{1 / 3}\right)+s\left\langle\mathbf{1}_{m+n}, \mathbb{E}[\mathbf{q}]\right\rangle
$$

Now, using Lemma 1, we can upper bound the expected queue length as follows:

$$
\begin{aligned}
& \mathbb{E}\left[\left\langle\boldsymbol{\theta}, \mathbf{q}^{(2)}\right\rangle\right]+\mathbb{E}\left[\left\langle\boldsymbol{\phi}, \mathbf{q}^{(1)}\right\rangle\right] \leq \tau_{\max }^{\eta}\left(\sum_{j=1}^{m} \theta_{j} \mathbb{P}\left[q_{j}^{(2)}>\tau_{\max }^{\eta}\right]+\sum_{i=1}^{n} \phi_{i} \mathbb{P}\left[q_{i}^{(1)}>\tau_{\max }^{\eta}\right]\right) \\
&+\frac{\eta}{\sigma^{\eta}}\left(\left\langle\mathbf{1}_{n}, \boldsymbol{\mu}^{*}\right\rangle+\left\langle\mathbf{1}_{m}, \boldsymbol{\lambda}^{*}\right\rangle\right) \\
& \Rightarrow \min \{(\boldsymbol{\theta}, \boldsymbol{\phi})\} \mathbb{E}\left[\left\langle\mathbf{1}_{m+n}, \mathbf{q}\right\rangle\right] \leq \tau_{\max }^{\eta}\left(\sum_{j=1}^{m} \theta_{j}+\sum_{i=1}^{n} \phi_{i}\right)+\frac{\eta}{\sigma^{\eta}}\left(\left\langle\mathbf{1}_{n}, \boldsymbol{\mu}^{*}\right\rangle+\left\langle\mathbf{1}_{m}, \boldsymbol{\lambda}^{*}\right\rangle\right) \\
& \Rightarrow \min \{(\boldsymbol{\theta}, \boldsymbol{\phi})\} \mathbb{E}\left[\left\langle\mathbf{1}_{m+n}, \mathbf{q}\right\rangle\right] \stackrel{*}{=} O\left(\eta^{1 / 3}\right) \Rightarrow \mathbb{E}\left[\left\langle\mathbf{1}_{m+n}, \mathbf{q}\right\rangle\right] \stackrel{*}{=} O\left(\eta^{1 / 3}\right)
\end{aligned}
$$

where $(*)$ follows by substituting $\tau_{\max }^{\eta}=T_{1} \eta^{1 / 3}, \sigma^{\eta}=\eta^{2 / 3}$ and noting that $\min \{(\boldsymbol{\theta}, \boldsymbol{\phi})\}>0$. Finally by substituting (50) in (49) we have the theorem.

\section{Proof of Theorem 3}

First, we will present a lemma in which, we will lower bound the expected value of the sum of the queue length $\mathbb{E}\left[\left\langle\mathbf{1}_{n+m}, \mathbf{q}\right\rangle\right]$. 
Figure $10 \quad$ Coupled Birth and Death Process

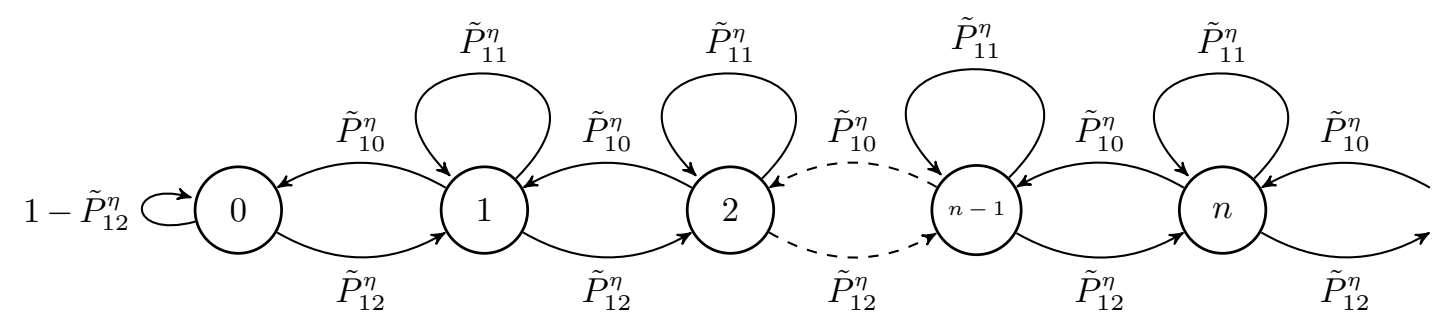

LEMma 4. If Condition 10 (a) is satisfied, then

$$
\mathbb{E}\left[\left\langle\mathbf{1}_{n+m}, \mathbf{q}\right\rangle\right] \geq \frac{\eta^{1-\beta}\left\langle\mathbf{1}_{m}, \boldsymbol{\lambda}^{*}\right\rangle}{2\left(\left\langle\mathbf{1}_{n}, \mathbf{N}\right\rangle+\left\langle\mathbf{1}_{m}, \mathbf{M}\right\rangle\right)}-0.5
$$

In addition, if Condition 1 (b) and (c) are satisfied, then there exists $\epsilon>0$ and $\mathcal{M}>0$ such that

$$
\sum_{j=1}^{m} \mathbb{E}\left[f_{j}^{2}\left(\frac{\mathbf{q}}{\eta^{\boldsymbol{\alpha}}}\right)\right]+\sum_{i=1}^{n} \mathbb{E}\left[g_{i}^{2}\left(\frac{\mathbf{q}}{\eta^{\boldsymbol{\alpha}}}\right)\right] \geq \epsilon \forall \eta>\mathcal{M}
$$

Proof of Lemma 4 First, recall that we denote the imbalance for a CTMC by $z$ which is defined in (15). Now, we will define a new DTMC $\left\{\tilde{z}^{\eta}(k): k \in \mathbb{Z}_{+}\right\}$and couple it with the Uniformized $\operatorname{DTMC}\left\{\mathbf{q}^{\eta}(k), z^{\eta}(k): k \in \mathbb{Z}_{+}\right\}$with uniformization constant $c$ such that $\left|z^{\eta}(k)\right| \geq \tilde{z}^{\eta}(k)$ for all $k \in \mathbb{Z}_{+}$for all $\eta \geq 1$ if $z(0)=\tilde{z}(0)$.

The state space of $\left\{\tilde{z}^{\eta}(k): k \in \mathbb{Z}_{+}\right\}$is $\mathbb{Z}_{+}$and the transition matrix is given by

$$
\tilde{P}_{i j}^{\eta}= \begin{cases}\tilde{P}_{10}^{\eta} \triangleq\left(\left\langle\mathbf{1}_{n}, \boldsymbol{\mu}^{*}\right\rangle+\left(\left\langle\mathbf{1}_{n}, \mathbf{N}\right\rangle+\left\langle\mathbf{1}_{m}, \mathbf{M}\right\rangle\right) \eta^{\beta-1}\right) / c & \text { if } j=i-1 \forall j \in \mathbb{Z}_{+}, \forall i>0 \\ \tilde{P}_{12}^{\eta} \triangleq\left(\left\langle\mathbf{1}_{m}, \boldsymbol{\lambda}^{*}\right\rangle-\left(\left\langle\mathbf{1}_{n}, \mathbf{N}\right\rangle+\left\langle\mathbf{1}_{m}, \mathbf{M}\right\rangle\right) \eta^{\beta-1}\right) / c & \text { if } j=i+1 \forall j \in \mathbb{Z}_{+}, \forall i>0 \\ \tilde{P}_{11}^{\eta} \triangleq 1-\left(\left\langle\mathbf{1}_{n}, \boldsymbol{\mu}^{*}\right\rangle+\left\langle\mathbf{1}_{m}, \boldsymbol{\lambda}^{*}\right\rangle\right) / c & \text { if } j=i \forall j \in \mathbb{Z}_{+}, \forall i>0 \\ \left(\left\langle\mathbf{1}_{m}, \boldsymbol{\lambda}^{*}\right\rangle-\left(\left\langle\mathbf{1}_{n}, \mathbf{N}\right\rangle+\left\langle\mathbf{1}_{m}, \mathbf{M}\right\rangle\right) \eta^{\beta-1}\right) / c & \text { if } j=1, i=0 \\ 1-\left(\left\langle\mathbf{1}_{m}, \boldsymbol{\lambda}^{*}\right\rangle-\left(\left\langle\mathbf{1}_{n}, \mathbf{N}\right\rangle+\left\langle\mathbf{1}_{m}, \mathbf{M}\right\rangle\right) \eta^{\beta-1}\right) / c & \text { if } j=i=0 \\ 0 & \text { otherwise. }\end{cases}
$$

where $c$ is the uniformization constant given by (2). Note that, under condition 10 (a) we have $\tilde{P}_{i j}^{\eta} \geq P[|z(k+1)|=j|| z(k) \mid=i, \mathbf{q}(k)=\overline{\mathbf{q}}]$ for all $\overline{\mathbf{q}} \in S$ if $j<i$ and $\tilde{P}_{i j}^{\eta} \leq P[|z(k+1)|=j|| z(k) \mid=$ $i, \mathbf{q}(k)=\overline{\mathbf{q}}]$ for all $\overline{\mathbf{q}} \in S$ if $j>i$ for all $\eta>0$. Thus, we can couple these system using a common source of randomness, such that $\tilde{z}(k) \leq|z(k)|$ for all $k \in \mathbb{Z}_{+}$sample path wise. Thus, we have $\mathbb{P}[\tilde{z}(k) \leq \mathcal{K}] \geq \mathbb{P}[z(k) \leq \mathcal{K}]$ for all $k \geq 1$ and $\mathcal{K} \in \mathbb{R}$ Thus, in the limit as $k \rightarrow \infty$, we have $\mathbb{P}[\tilde{z}(\infty) \leq$ $\mathcal{K}] \geq \mathbb{P}[z(\infty) \leq \mathcal{K}]$ where, $\tilde{z}(\infty)$ and $z(\infty)$ denotes a random variable with distribution same as the stationary distribution of $\{\tilde{z}(k): k \geq 1\}$ and $\{z(k): k \geq 1\}$ respectively and so, we have

$$
\mathbb{E}[\tilde{z}(\infty)] \leq \mathbb{E}[|z(\infty)|]
$$


Next, the stationary distribution of $\{\tilde{z}(k): k \geq 1\}$ is given by

$$
\begin{aligned}
\pi_{i} & =\pi_{0}\left(\frac{\tilde{P}_{12}^{\eta}}{\tilde{P}_{10}^{\eta}}\right)^{i} \forall i>0 \\
\sum_{i=-\infty}^{\infty} \pi_{i} & =1
\end{aligned}
$$

Solving for $\pi_{i}$, we get

$$
\pi_{i}=\left(1-\frac{\tilde{P}_{12}^{\eta}}{\tilde{P}_{10}^{\eta}}\right)\left(\frac{\tilde{P}_{12}^{\eta}}{\tilde{P}_{10}^{\eta}}\right)^{i} \forall i \in \mathbb{Z}_{+}
$$

Thus, we have

$$
\begin{aligned}
\mathbb{E}[\tilde{z}(\infty)] & =\frac{\tilde{P}_{12}^{\eta}}{\tilde{P}_{10}^{\eta}-\tilde{P}_{12}^{\eta}} \\
& \stackrel{*}{=} \frac{\eta^{1-\beta}\left\langle\mathbf{1}_{m}, \boldsymbol{\lambda}^{*}\right\rangle}{2\left(\left\langle\mathbf{1}_{n}, \mathbf{N}\right\rangle+\left\langle\mathbf{1}_{m}, \mathbf{M}\right\rangle\right)}-0.5 .
\end{aligned}
$$

where, $\left\langle\mathbf{1}_{n}, \boldsymbol{\mu}^{*}\right\rangle=\left\langle\mathbf{1}_{m}, \boldsymbol{\lambda}^{*}\right\rangle$ is used to conclude $(*)$ which holds as we have $\left(\boldsymbol{\lambda}^{*}, \boldsymbol{\mu}^{*}\right) \in \mathbb{C}$. Finally, note that $|z| \leq\left\langle\mathbf{1}_{n+m}, \mathbf{q}\right\rangle$. Thus, we have

$$
\mathbb{E}\left[\left\langle\mathbf{1}_{n+m}, \mathbf{q}\right\rangle\right] \geq \mathbb{E}[|z(\infty)|] \stackrel{\sqrt{53})}{\geq} \mathbb{E}[\tilde{z}(\infty)] \stackrel{(55)}{=} \frac{\eta^{1-\beta}\left\langle\mathbf{1}_{m}, \boldsymbol{\lambda}^{*}\right\rangle}{2\left(\left\langle\mathbf{1}_{n}, \mathbf{N}\right\rangle+\left\langle\mathbf{1}_{m}, \mathbf{M}\right\rangle\right)}-0.5,
$$

which completes the first part of the proof. Next, as $\tilde{z}(\infty) \leq|z(\infty)|$ almost surely, for any $\mathcal{K}$, we have

$$
\mathbb{P}[|z(\infty)|>\mathcal{K}] \geq \mathbb{P}[\tilde{z}(\infty)>\mathcal{K}]=\sum_{i=\mathcal{K}+1}^{\infty} \pi_{i}=\left(\frac{\tilde{P}_{12}^{\eta}}{\tilde{P}_{10}^{\eta}}\right)^{\mathcal{K}+1}=\left(\frac{\left\langle\mathbf{1}_{m}, \boldsymbol{\lambda}^{*}\right\rangle-\left(\left\langle\mathbf{1}_{n}, \mathbf{N}\right\rangle+\left\langle\mathbf{1}_{m}, \mathbf{M}\right\rangle\right) \eta^{\beta-1}}{\left\langle\mathbf{1}_{n}, \boldsymbol{\mu}^{*}\right\rangle+\left(\left\langle\mathbf{1}_{n}, \mathbf{N}\right\rangle+\left\langle\mathbf{1}_{m}, \mathbf{M}\right\rangle\right) \eta^{\beta-1}}\right)^{\mathcal{K}+1}
$$

Now, for $\mathcal{K}=(n+m) K \eta^{\alpha}$ first note that when $\alpha=0$, we have

$$
\lim _{\eta \rightarrow \infty}\left(\frac{\left\langle\mathbf{1}_{m}, \boldsymbol{\lambda}^{*}\right\rangle-\left(\left\langle\mathbf{1}_{n}, \mathbf{N}\right\rangle+\left\langle\mathbf{1}_{m}, \mathbf{M}\right\rangle\right) \eta^{\beta-1}}{\left\langle\mathbf{1}_{n}, \boldsymbol{\mu}^{*}\right\rangle+\left(\left\langle\mathbf{1}_{n}, \mathbf{N}\right\rangle+\left\langle\mathbf{1}_{m}, \mathbf{M}\right\rangle\right) \eta^{\beta-1}}\right)^{(n+m) K+1}=1^{(n+m) K+1}=1 .
$$

Now, for $\alpha>0$, we have

$$
\begin{aligned}
& \lim _{\eta \rightarrow \infty}\left(\frac{\left\langle\mathbf{1}_{m}, \boldsymbol{\lambda}^{*}\right\rangle-\left(\left\langle\mathbf{1}_{n}, \mathbf{N}\right\rangle+\left\langle\mathbf{1}_{m}, \mathbf{M}\right\rangle\right) \eta^{\beta-1}}{\left\langle\mathbf{1}_{n}, \boldsymbol{\mu}^{*}\right\rangle+\left(\left\langle\mathbf{1}_{n}, \mathbf{N}\right\rangle+\left\langle\mathbf{1}_{m}, \mathbf{M}\right\rangle\right) \eta^{\beta-1}}\right)^{(n+m) K \eta^{\alpha}} \\
= & \lim _{\eta \rightarrow \infty}\left[\left(1-\frac{2\left(\left\langle\mathbf{1}_{n}, \mathbf{N}\right\rangle+\left\langle\mathbf{1}_{m}, \mathbf{M}\right\rangle\right)}{\left\langle\mathbf{1}_{m}, \boldsymbol{\mu}^{*}\right\rangle \eta^{1-\beta}+\left(\left\langle\mathbf{1}_{n}, \mathbf{N}\right\rangle+\left\langle\mathbf{1}_{m}, \mathbf{M}\right\rangle\right)}\right)^{\frac{\left\langle\mathbf{1}_{n}, \boldsymbol{\mu}^{*}\right\rangle \eta^{1-\beta}}{3\left(\left\langle\mathbf{1}_{n}, \mathbf{N}\right\rangle+\left\langle\mathbf{1}_{m}, \mathbf{M}\right\rangle\right)}}\right]^{\frac{3 K(n+m)\left(\left\langle\mathbf{1}_{n}, \mathbf{N}\right\rangle+\left\langle\mathbf{1}_{m}, \mathbf{M}\right\rangle\right)}{\left\langle\mathbf{1}_{n}, \boldsymbol{\mu}^{*}\right\rangle} \eta^{\alpha+\beta-1}} \\
\stackrel{2}{\geq} \lim _{\eta \rightarrow \infty}\left(1-\frac{2}{3+\frac{3\left(\left\langle\mathbf{1}_{n}, \mathbf{N}\right\rangle+\left\langle\mathbf{1}_{m}, \mathbf{M}\right\rangle\right)}{\left\langle\mathbf{1}_{n}, \boldsymbol{\mu}^{*}\right\rangle} \eta^{\beta-1}}\right)^{\frac{3 K(n+m)\left(\left\langle\mathbf{1}_{n}, \mathbf{N}\right\rangle+\left\langle\mathbf{1}_{m}, \mathbf{M}\right\rangle\right)}{\left\langle\mathbf{1}_{n}, \boldsymbol{\mu}^{*}\right\rangle} \eta^{\alpha+\beta-1}} & \text { if } \alpha+\beta-1<0 \\
= & \begin{cases}(1 / 3)^{0}=1 & \text { if } \alpha+\beta=1 \\
b \triangleq\left(\frac{1}{3}\right)^{\frac{3 K(n+m)\left(\left\langle\mathbf{1}_{n}, \mathbf{N}\right\rangle+\left\langle\mathbf{1}_{m}, \mathbf{M}\right\rangle\right)}{\left\langle\mathbf{1}_{n}, \boldsymbol{\mu}^{*}\right\rangle}}\end{cases}
\end{aligned}
$$


where $(*)$ follows from the Bernoulli's inequality which says that $(1+x)^{r} \geq 1+r x$ if $x \geq-2$ and thus, the inequality follows by comparison theorem for sequences. Thus, we have

$$
\begin{aligned}
& \lim _{\eta \rightarrow \infty}\left(\frac{\left\langle\mathbf{1}_{m}, \boldsymbol{\lambda}^{*}\right\rangle-\left(\left\langle\mathbf{1}_{n}, \mathbf{N}\right\rangle+\left\langle\mathbf{1}_{m}, \mathbf{M}\right\rangle\right) \eta^{\beta-1}}{\left\langle\mathbf{1}_{n}, \boldsymbol{\mu}^{*}\right\rangle+\left(\left\langle\mathbf{1}_{n}, \mathbf{N}\right\rangle+\left\langle\mathbf{1}_{m}, \mathbf{M}\right\rangle\right) \eta^{\beta-1}}\right) \\
& \stackrel{*}{=} \lim _{\eta \rightarrow \infty}\left(\frac{\left\langle\mathbf{1}_{m}, \boldsymbol{\lambda}^{*}\right\rangle-\left(\left\langle\mathbf{1}_{n}, \mathbf{N}\right\rangle+\left\langle\mathbf{1}_{m}, \mathbf{M}\right\rangle\right) \eta^{\beta-1}}{\left\langle\mathbf{1}_{n}, \boldsymbol{\mu}^{*}\right\rangle+\left(\left\langle\mathbf{1}_{n}, \mathbf{N}\right\rangle+\left\langle\mathbf{1}_{m}, \mathbf{M}\right\rangle\right) \eta^{\alpha-1}}\right)^{(n+m) K \eta^{\alpha}} \times \lim _{\eta \rightarrow \infty}\left(\frac{\left\langle\mathbf{1}_{m}, \boldsymbol{\lambda}^{*}\right\rangle-\left(\left\langle\mathbf{1}_{n}, \mathbf{N}\right\rangle+\left\langle\mathbf{1}_{m}, \mathbf{M}\right\rangle\right) \eta^{\beta-1}}{\left\langle\mathbf{1}_{n}, \boldsymbol{\mu}^{*}\right\rangle+\left(\left\langle\mathbf{1}_{n}, \mathbf{N}\right\rangle+\left\langle\mathbf{1}_{m}, \mathbf{M}\right\rangle\right) \eta^{\beta-1}}\right) \\
& = \begin{cases}1 & \text { if } \alpha+\beta-1<0 \\
b & \text { if } \alpha+\beta=1\end{cases}
\end{aligned}
$$

where $(*)$ follows as the limit of product of two sequences is the product of limit of the two sequences. Thus, when Condition 1) (b) is satisfied, that is $\alpha+\beta \leq 1$, for all $b \delta^{2}>\epsilon>0$ there exists an $\mathcal{M}$ such that for all $\eta>\mathcal{M}$, we have

$$
P\left[|z(\infty)|>(n+m) K \eta^{\alpha}\right] \geq \frac{\epsilon}{\delta^{2}}
$$

Also note that, by the definition of $z$, we have

$$
\left\{|z|>(n+m) K \eta^{\alpha}\right\} \subset\left\{\|\mathbf{q}\|_{\infty}>K \eta^{\alpha}\right\}
$$

Also, by Condition $1(\mathrm{~b})$, we have

$$
\sum_{j=1}^{m} f_{j}^{2}\left(\frac{\mathbf{q}}{\eta^{\boldsymbol{\alpha}}}\right)+\sum_{i=1}^{n} g_{i}^{2}\left(\frac{\mathbf{q}}{\eta^{\boldsymbol{\alpha}}}\right) \geq \delta^{2} \forall \mathbf{q}:\|\mathbf{q}\|_{\infty}>k \eta^{\alpha}
$$

Thus for all $\eta>\mathcal{M}$, we have

$$
\begin{aligned}
\sum_{j=1}^{m} \mathbb{E}\left[f_{j}^{2}\left(\frac{\mathbf{q}}{\eta^{\alpha}}\right)\right]+\sum_{i=1}^{n} \mathbb{E}\left[g_{i}^{2}\left(\frac{\mathbf{q}}{\eta^{\alpha}}\right)\right] & \geq \delta^{2} \mathbb{P}\left[|z(\infty)|>(n+m) K \eta^{\alpha}\right] \\
& \geq \delta^{2} \frac{\epsilon}{\delta^{2}} \\
& =\epsilon .
\end{aligned}
$$

We will now prove Theorem 3 .

Proof of Theorem 3 Without loss of generality, we can assume that

$$
\mathbb{E}\left[\left\langle\mathbf{1}_{n+m}, \mathbf{q}\right\rangle\right]<\infty
$$

since, otherwise, it will result in infinite profit loss. Now we will calculate the profit loss and use the queue length bound given by Lemma 4 to lower bound it. We have 


$$
\begin{aligned}
& =\eta\left\langle F\left(\boldsymbol{\lambda}^{*}\right), \boldsymbol{\lambda}^{*}\right\rangle-\eta\left\langle G\left(\boldsymbol{\mu}^{*}\right), \boldsymbol{\mu}^{*}\right\rangle-\mathbb{E}\left[\left\langle F^{\eta}\left(\boldsymbol{\lambda}^{\eta}\right), \boldsymbol{\lambda}^{\eta}\right\rangle-\left\langle G^{\eta}\left(\boldsymbol{\mu}^{\eta}\right), \boldsymbol{\mu}^{\eta}\right\rangle-s\left\langle\mathbf{1}_{n+m}, \mathbf{q}\right\rangle\right] \\
& \stackrel{(a)}{=} \eta\left\langle F\left(\boldsymbol{\lambda}^{*}\right), \boldsymbol{\lambda}^{*}\right\rangle-\eta\left\langle G\left(\boldsymbol{\mu}^{*}\right), \boldsymbol{\mu}^{*}\right\rangle-\mathbb{E}\left[\sum_{j=1}^{m}\left(\lambda_{j}^{*}+f_{j}\left(\frac{\mathbf{q}}{\eta^{\alpha}} \eta^{\beta-1}\right)\right) F_{j}\left(\lambda_{j}^{*}+f_{j}\left(\frac{\mathbf{q}}{\eta^{\alpha}} \eta^{\beta-1}\right)\right)\right. \\
& \left.\quad-\sum_{i=1}^{n}\left(\mu_{i}^{*}+g_{i}\left(\frac{\mathbf{q}}{\eta^{\alpha}}\right) \eta^{\beta-1}\right) G_{i}\left(\mu_{i}^{*}+g_{i}\left(\frac{\mathbf{q}}{\eta^{\alpha}}\right) \eta^{\beta-1}\right)-s\left\langle\mathbf{1}_{n+m}, \mathbf{q}\right\rangle\right],
\end{aligned}
$$

where $(a)$ follows from the definition of asymptotic regime (Definition 3 ). Now, we will use Taylor's Theorem for each function $F_{j}$ and $G_{i}$ individually. For example, we have

$$
\begin{aligned}
& \mathbb{E}\left[\left(\eta \lambda_{j}^{*}+f_{j}\left(\frac{\mathbf{q}}{\eta^{\alpha}}\right) \eta^{\beta}\right) F_{j}\left(\lambda_{j}^{*}+f_{j}\left(\frac{\mathbf{q}}{\eta^{\alpha}}\right) \frac{\eta^{\beta}}{\eta}\right)\right] \\
\stackrel{(b)}{=} & \mathbb{E}\left[\left(\eta \lambda_{j}^{*}+f_{j}\left(\frac{\mathbf{q}}{\eta^{\alpha}}\right) \eta^{\beta}\right)\left(F_{j}\left(\lambda_{j}^{*}\right)+f_{j}\left(\frac{\mathbf{q}}{\eta^{\alpha}}\right) \eta^{\beta-1} F_{j}^{\prime}\left(\lambda_{j}^{*}\right)+f_{j}^{2}\left(\frac{\mathbf{q}}{\eta^{\alpha}}\right) \frac{\eta^{2 \beta-2}}{2} F_{j}^{\prime \prime}\left(\tilde{\lambda}_{j}(\mathbf{q})\right)\right)\right] \\
= & \eta \lambda_{j}^{*} F_{j}\left(\lambda_{j}^{*}\right)+\left(F_{j}^{\prime}\left(\lambda_{j}^{*}\right) \lambda_{j}^{*}+F_{j}\left(\lambda_{j}^{*}\right)\right) \mathbb{E}\left[f_{j}\left(\frac{\mathbf{q}}{\eta^{\alpha}}\right)\right] \eta^{\beta}+F_{j}^{\prime}\left(\lambda_{j}^{*}\right) \mathbb{E}\left[f_{j}^{2}\left(\frac{\mathbf{q}}{\eta^{\alpha}}\right)\right] \eta^{2 \beta-1} \\
& +\lambda_{j}^{*} \mathbb{E}\left[f_{j}^{2}\left(\frac{\mathbf{q}}{\eta^{\alpha}}\right) F_{j}^{\prime \prime}\left(\tilde{\lambda}_{j}(\mathbf{q})\right)\right] \frac{\eta^{2 \beta-1}}{2}+\mathbb{E}\left[f_{j}^{3}\left(\frac{\mathbf{q}}{\eta^{\alpha}}\right) F_{j}^{\prime \prime}\left(\tilde{\lambda}_{j}(\mathbf{q})\right)\right] \frac{\eta^{3 \beta-2}}{2},
\end{aligned}
$$

where (b) follows from Taylor's theorem and $\tilde{\lambda}_{j}(\mathbf{q}) \in\left[\lambda_{j}^{*}, \lambda_{j}^{*}+f_{j}\left(\frac{\mathbf{q}}{\eta^{\alpha}}\right) \eta^{\beta-1}\right]$. Similarly, using the Taylor's theorem for all $F_{j}$ and $G_{i}$ in (57), for some $\tilde{\lambda}_{j}(\mathbf{q}) \in\left[\lambda_{j}^{*}, \lambda_{j}^{*}+f_{j}\left(\frac{\mathbf{q}}{\eta^{\alpha}}\right) \eta^{\beta-1}\right]$ for all $j \in[m]$ and $\tilde{\mu}_{i}(\mathbf{q}) \in\left[\mu_{i}^{*}, \mu_{i}^{*}+g_{i}\left(\frac{\mathbf{q}}{\eta^{\alpha}}\right) \eta^{\beta-1}\right]$ for all $i \in[n]$, we have

$$
\begin{aligned}
L^{\eta}= & \underbrace{\left.\left.\sum_{i=1}^{n} \mathbb{E}\left[g_{i}\left(\frac{\mathbf{q}}{\eta^{\alpha}}\right) \eta^{\beta}\right]\left(\mu_{i}^{*} G_{i}^{\prime}\left(\mu_{i}^{*}\right)+G_{i}\left(\mu_{i}^{*}\right)\right)\right)-\sum_{j=1}^{m} \mathbb{E}\left[f_{j}\left(\frac{\mathbf{q}}{\eta^{\alpha}}\right) \eta^{\beta}\right]\left(\lambda_{j}^{*} F_{j}^{\prime}\left(\lambda_{j}^{*}\right)+F_{j}\left(\lambda_{j}^{*}\right)\right)\right)}_{\mathcal{A}_{1}} \\
& \underbrace{-\eta^{2 \beta-1}\left(\sum_{j=1}^{m} F_{j}^{\prime}\left(\lambda_{j}^{*}\right) \mathbb{E}\left[f_{j}^{2}\left(\frac{\mathbf{q}}{\eta^{\alpha}}\right)\right]+\sum_{j=1}^{m} \frac{\lambda_{j}^{*}}{2} \mathbb{E}\left[F_{j}^{\prime \prime}\left(\tilde{\lambda}_{j}(\mathbf{q})\right) f_{j}^{2}\left(\frac{\mathbf{q}}{\eta^{\alpha}}\right)\right]\right)}_{\mathcal{A}_{2}^{(2)}} \\
& \underbrace{+\eta^{2 \beta-1}\left(\sum_{i=1}^{n} G_{i}^{\prime}\left(\mu_{i}^{*}\right) \mathbb{E}\left[g_{i}^{2}\left(\frac{\mathbf{q}}{\eta^{\alpha}}\right)\right]+\sum_{i=1}^{n} \frac{\mu_{i}^{*}}{2} \mathbb{E}\left[G_{i}^{\prime \prime}(\tilde{\mu}(\mathbf{q})) g_{i}^{2}\left(\frac{\mathbf{q}}{\eta^{\alpha}}\right)\right]\right)}_{\mathcal{A}_{2}^{(1)}} \\
& \underbrace{-\frac{\eta^{3 \beta-2}}{2}\left(\sum_{j=1}^{m} \mathbb{E}\left[F_{j}^{\prime \prime}\left(\tilde{\lambda}_{j}(\mathbf{q})\right) f_{j}^{3}\left(\frac{\mathbf{q}}{\eta^{\alpha}}\right)\right]-\sum_{i=1}^{n} \mathbb{E}\left[G_{i}^{\prime \prime}\left(\tilde{\mu}_{i}(\mathbf{q})\right) g_{i}^{3}\left(\frac{\mathbf{q}}{\eta^{\alpha}}\right)\right]\right)}_{\mathcal{A}_{3}}+\underbrace{s \mathbb{E}^{\alpha}\left[\left\langle\mathbf{1}_{n+m}, \mathbf{q}\right\rangle\right]}_{\mathcal{A}_{4}}
\end{aligned}
$$

Now, we will lower bound each of these terms individually. Firstly, we have $\mathcal{A}_{1} \geq 0$ by Claim 2 and (56). Next, $\mathcal{A}_{2}^{(1)}$ and $\mathcal{A}_{2}^{(2)}$ can be simplified using the following claim:

Claim 3. We have

$$
\begin{gathered}
A_{j} \triangleq-F_{j}^{\prime}\left(\lambda_{j}^{*}\right)-\frac{1}{2} \lambda_{j}^{*} F_{j}^{\prime \prime}\left(\lambda_{j}^{*}\right)>0, \forall j \in[m] \\
B_{i} \triangleq G_{i}^{\prime}\left(\mu_{i}^{*}\right)+\frac{1}{2} \mu_{i}^{*} G_{i}^{\prime \prime}\left(\mu_{i}^{*}\right)>0 \forall i \in[n] .
\end{gathered}
$$


Also, there exists $\mathcal{M}^{\prime}>0$ such that for all $\eta>\mathcal{M}^{\prime}$ and $\tilde{c} \triangleq \min _{j \in[m], i \in[n]}\left\{\frac{A_{j}}{\lambda_{j}^{*}}, \frac{B_{i}}{\mu_{i}^{*}}\right\}$, we have

$$
\begin{aligned}
& \sup _{\mathbf{q} \in S}\left|F_{j}^{\prime \prime}\left(\tilde{\lambda}_{j}(\mathbf{q})\right)-F_{j}^{\prime \prime}\left(\lambda_{j}^{*}\right)\right|<\tilde{c} . \forall j \in[m] \\
& \sup _{\mathbf{q} \in S}\left|G_{i}^{\prime \prime}\left(\tilde{\mu}_{i}(\mathbf{q})\right)-G_{j}^{\prime \prime}\left(\mu_{i}^{*}\right)\right|<\tilde{c} . \forall i \in[n]
\end{aligned}
$$

The proof is deferred to the end of this section. Using Claim [3, we have

$$
\begin{aligned}
\mathcal{A}_{2}^{(2)}+\mathcal{A}_{2}^{(1)} & \geq \eta^{2 \beta-1}\left(\sum_{j=1}^{m}\left(A_{j}-\frac{\lambda_{j}^{*} \tilde{c}}{2}\right) \mathbb{E}\left[f_{j}^{2}\left(\frac{\mathbf{q}}{\eta^{\alpha}}\right)\right]+\sum_{i=1}^{n}\left(B_{i}-\frac{\mu_{i}^{*} \tilde{c}}{2}\right) \mathbb{E}\left[g_{i}^{2}\left(\frac{\mathbf{q}}{\eta^{\alpha}}\right)\right]\right) \\
& \geq \eta^{2 \beta-1}\left(\sum_{j=1}^{m} \frac{A_{j}}{2} \mathbb{E}\left[f_{j}^{2}\left(\frac{\mathbf{q}}{\eta^{\alpha}}\right)\right]+\sum_{i=1}^{n} \frac{B_{i}}{2} \mathbb{E}\left[g_{i}^{2}\left(\frac{\mathbf{q}}{\eta^{\alpha}}\right)\right]\right) \text { (Definition of } \tilde{c} \text { ) }
\end{aligned}
$$

Next, we have

$$
\begin{aligned}
\mathcal{A}_{3} & =-\frac{\eta^{3 \beta-2}}{2}\left(\sum_{j=1}^{m} \mathbb{E}\left[F_{j}^{\prime \prime}\left(\tilde{\lambda}_{j}(\mathbf{q})\right) f_{j}^{3}\left(\frac{\mathbf{q}}{\eta^{\boldsymbol{\alpha}}}\right)\right]-\sum_{i=1}^{n} \mathbb{E}\left[G_{i}^{\prime \prime}\left(\tilde{\mu}_{i}(\mathbf{q})\right) g_{i}^{3}\left(\frac{\mathbf{q}}{\eta^{\boldsymbol{\alpha}}}\right)\right]\right) \\
& \geq-\eta^{3 \beta-2} \frac{\left\langle\mathbf{1}_{m},\left|F^{\prime \prime}\left(\boldsymbol{\lambda}^{*}\right)\right|\right\rangle+\left\langle\mathbf{1}_{n},\left|G^{\prime \prime}\left(\boldsymbol{\mu}^{*}\right)\right|\right\rangle+2 \tilde{c}}{2}\left(\mathbb{E}\left[\left|f^{3}\left(\frac{\mathbf{q}}{\eta^{\alpha}}\right)\right|\right]+\mathbb{E}\left[\left|g^{3}\left(\frac{\mathbf{q}}{\eta^{\alpha}}\right)\right|\right]\right)
\end{aligned}
$$

Next, as $\beta<1$, for all $\epsilon^{\prime \prime}>0$ there exists $\mathcal{M}^{\prime \prime}>0$ such that for all $\eta>\mathcal{M}^{\prime \prime}$, we have

$$
\frac{\mathcal{A}_{3}}{\eta^{2 \beta-1}}>-\epsilon^{\prime \prime}\left(\sum_{j=1}^{m} \mathbb{E}\left[\left|f_{j}^{3}\left(\frac{\mathbf{q}}{\eta^{\alpha}}\right)\right|\right]+\sum_{i=1}^{n} \mathbb{E}\left[\left|g_{i}^{3}\left(\frac{\mathbf{q}}{\eta^{\alpha}}\right)\right|\right]\right) \text {. }
$$

Finally, $\mathcal{A}_{4}$ can be lower bounded using Lemma 4. Combining everything, for all $\eta>\mathcal{M} \triangleq$ $\max \left\{\mathcal{M}^{\prime}, \mathcal{M}^{\prime \prime}\right\}$, we have

$$
\begin{aligned}
L^{\eta} \geq & \eta^{2 \beta-1}\left(\sum_{j=1}^{m} \frac{A_{j}}{2} \mathbb{E}\left[f_{j}^{2}\left(\frac{\mathbf{q}}{\eta^{\alpha}}\right)\right]+\sum_{i=1}^{n} \frac{B_{i}}{2} \mathbb{E}\left[g_{i}^{2}\left(\frac{\mathbf{q}}{\eta^{\alpha}}\right)\right]\right) \\
& -\eta^{2 \beta-1} \epsilon^{\prime \prime}\left(\sum_{j=1}^{m} \mathbb{E}\left[\left|f_{j}^{3}\left(\frac{\mathbf{q}}{\eta^{\alpha}}\right)\right|\right]+\sum_{i=1}^{n} \mathbb{E}\left[\left|g_{i}^{3}\left(\frac{\mathbf{q}}{\eta^{\alpha}}\right)\right|\right]\right)+s \mathbb{E}\left[\left\langle\mathbf{1}_{n+m}, \mathbf{q}\right\rangle\right]
\end{aligned}
$$

Now, pick $\epsilon^{\prime \prime}=\frac{\min _{i, j}\left\{A_{j}, B_{i}\right\} \epsilon}{4\left(M^{3}+N^{3}\right)}$ and using Lemma 4 and Condition 1 (a) we get

$$
\begin{aligned}
& L^{\eta} \geq \eta^{2 \beta-1}\left(\frac{\min _{i, j}\left\{A_{j}, B_{i}\right\} \epsilon}{2}-\frac{\min _{i, j}\left\{A_{j}, B_{i}\right\} \epsilon}{4}\right)+\frac{s \eta^{1-\beta}\left\langle\mathbf{1}_{m}, \boldsymbol{\lambda}^{*}\right\rangle}{2\left(\left\langle\mathbf{1}_{n}, \mathbf{N}\right\rangle+\left\langle\mathbf{1}_{m}, \mathbf{M}\right\rangle\right)}-0.5 s \\
& \quad \stackrel{(d)}{\geq} \inf _{\beta<1}\left\{\eta^{2 \beta-1} \frac{\min _{i, j}\left\{A_{j}, B_{i}\right\} \epsilon}{4}+\frac{s \eta^{1-\beta}\left\langle\mathbf{1}_{m}, \boldsymbol{\lambda}^{*}\right\rangle}{2\left(\left\langle\mathbf{1}_{n}, \mathbf{N}\right\rangle+\left\langle\mathbf{1}_{m}, \mathbf{M}\right\rangle\right)}-0.5 s\right\} \stackrel{(d)}{=} \Omega\left(\eta^{1 / 3}\right)
\end{aligned}
$$

where $(d)$ follows as the coefficient of the terms $\eta^{2 \beta-1}$ and $\eta^{1-\beta}$ are strictly positive and the minimum is achieved when $2 \beta-1=1-\beta$. 
Proof of the Claim 3 Recall that

$$
\begin{aligned}
& A_{j} \triangleq-F_{j}^{\prime}\left(\lambda_{j}^{*}\right)-\frac{1}{2} \lambda_{j}^{*} F_{j}^{\prime \prime}\left(\lambda_{j}^{*}\right), \forall j \in[m] \\
& B_{i} \triangleq G_{i}^{\prime}\left(\mu_{i}^{*}\right)+\frac{1}{2} \mu_{i}^{*} G_{i}^{\prime \prime}\left(\mu_{i}^{*}\right) \forall i \in[n] .
\end{aligned}
$$

As $F_{j}\left(\lambda_{j}\right) \lambda_{j}$ for all $j \in[m]$ and $-G_{i}\left(\mu_{i}\right) \mu_{i}$ for all $i \in[n]$ are concave by Assumption 2, we have

$$
\frac{d^{2}}{d \lambda_{j}^{2}}\left(F_{j}\left(\lambda_{j}\right) \lambda_{j}\right)=A_{j}>0 \forall j \in[m], \frac{d^{2}}{d \mu_{i}^{2}}\left(G_{i}\left(\mu_{i}\right) \mu_{i}\right)=B_{i}>0 \quad \forall i \in[n] .
$$

The strict inequality holds true as $F_{j}$ and $G_{i}$ for all $j \in[m]$ and $i \in[n]$ are strictly increasing by Assumption 1. Thus, the double derivative of $\lambda_{j} F_{j}\left(\lambda_{j}\right)$ for any $j \in[m]$ and $\mu_{i} G_{i}\left(\mu_{i}\right)$ for any $i \in[n]$ cannot be zero anywhere.

Now, as $F_{j}^{\prime \prime}($.$) is continuous, thus given \tilde{c}=\min _{i, j}\left\{\frac{A_{j}}{\lambda_{j}^{*}}, \frac{B_{i}}{\mu_{i}^{*}}\right\}>0$, there exists $\delta^{\prime}>0$ such that for $l \in\left[\lambda_{j}^{*}-\delta^{\prime}, \lambda_{j}^{*}+\delta^{\prime}\right]$ we have

$$
\left|F_{j}^{\prime \prime}(l)-F_{j}^{\prime \prime}\left(\lambda_{j}^{*}\right)\right|<\tilde{c}
$$

As $\beta<1$, we have $\eta^{\beta-1} \rightarrow 0$. Consider $\mathcal{M}^{\prime}$ such that for all $\eta>\mathcal{M}^{\prime}$ we have $\left|\eta^{\beta-1}\right|<$ $\delta^{\prime} / \max _{j \in[m], i \in[n]}\left\{M_{j}, N_{i}\right\}$ which implies that $\tilde{\lambda}_{j}(\mathbf{q}) \in\left[\lambda_{j}^{*}-\delta^{\prime}, \lambda_{j}^{*}+\delta^{\prime}\right]$ for all $\mathbf{q}$. Thus,

$$
\sup _{\mathbf{q} \in S}\left|F_{j}^{\prime \prime}\left(\tilde{\lambda}_{j}(\mathbf{q})\right)-F_{j}^{\prime \prime}\left(\lambda_{j}^{*}\right)\right|<\tilde{c} .
$$

Similarly, $G_{i}^{\prime \prime}\left(\tilde{\mu}_{i}(\mathbf{q})\right)$ for all $i \in[n]$ converges uniformly to $G_{i}^{\prime \prime}\left(\mu_{i}^{*}\right)$ respectively.

\section{J. Proof of Proposition 4}

We will first present a lemma for this case analogous to the Lemma 4 .

Lemma 5. Under the hypothesis of Proposition 4, and in addition if $\alpha+\beta>1$, there exists $\tilde{c}_{1}>0$, $\tilde{c}_{2}>0$ such that for all $\eta \geq 1$ we have

$$
\begin{aligned}
\mathbb{E}\left[q^{(1)}+q^{(2)}\right] & \geq \max \left\{\frac{1}{2} \eta^{\alpha}, \frac{\eta^{1-\beta} \lambda^{*}}{2(\theta+\phi)}-0.5\right\} \\
\mathbb{E}\left[f^{2}\left(\frac{\mathbf{q}}{\eta^{\alpha}}\right)+g^{2}\left(\frac{\mathbf{q}}{\eta^{\alpha}}\right)\right] & \geq \tilde{c}_{1} \eta^{1-\alpha-\beta} \\
\mathbb{E}\left[\left|f^{3}\left(\frac{\mathbf{q}}{\eta^{\alpha}}\right)\right|+\left|g^{3}\left(\frac{\mathbf{q}}{\eta^{\alpha}}\right)\right|\right] & \leq \tilde{c}_{2} \eta^{1-\alpha-\beta}
\end{aligned}
$$

where $f\left(\mathbf{q} / \eta^{\alpha}\right)=-\theta \mathbb{1}_{\left\{q^{(2)} \geq \eta^{\alpha}\right\}}$ and $g\left(\mathbf{q} / \eta^{\alpha}\right)=-\phi \mathbb{1}_{\left\{q^{(1)} \geq \eta^{\alpha}\right\}}$.

Proof Recall that the two-price policy for a single link two sided queue is of the form

$$
\lambda^{\eta}(q)=\left\{\begin{array}{ll}
\eta \lambda^{*} & \text { if } q \leq\left\lceil\eta^{\alpha}\right\rceil \\
\eta \lambda^{*}-\theta \eta^{\beta} & \text { otherwise. }
\end{array} \mu^{\eta}(q)= \begin{cases}\eta \mu^{*} & \text { if } q \geq-\left\lceil\eta^{\alpha}\right\rceil \\
\eta \mu^{*}-\phi \eta^{\beta} & \text { otherwise }\end{cases}\right.
$$


where $q=q^{(2)}-q^{(1)}$ and also recall that as it is a single link two sided queue, we have $\min \left\{q^{(2)}, q^{(1)}\right\}=0$. Now, consider a birth and death process denoted by $\{\tilde{q}(t): t \geq 0\}$ with state space $\mathbb{Z}$ and only one communicating class $\left\{q:-\tau_{\max }^{\eta} \leq q \leq \tau_{\max }^{\eta}\right\}$ and transition rates given by

$$
\tilde{\lambda}^{\eta}(q)=\left\{\begin{array}{ll}
\eta \lambda^{*} & \text { if } q \leq\left\lceil\eta^{\alpha}\right\rceil \\
0 & \text { otherwise. }
\end{array} \tilde{\mu}^{\eta}(q)= \begin{cases}\eta \mu^{*} & \text { if } q \geq-\left\lceil\eta^{\alpha}\right\rceil \\
0 & \text { otherwise. }\end{cases}\right.
$$

As, we have $\tilde{\lambda}(q) \leq \lambda(q)$ and $\tilde{\mu}(q) \geq \mu(q)$ for all $q \geq 0$ and $\tilde{\lambda}(q) \geq \lambda(q)$ and $\tilde{\mu}(q) \leq \mu(q)$ for all $q \geq 0$, thus, using a common source of randomness, we can couple the two systems such that $|\tilde{q}(t)| \leq|q(t)|$ for all $t \geq 1$. Thus, we have $\mathbb{P}[|q(t)| \leq \mathcal{K}] \leq \mathbb{P}[|\tilde{q}(t)| \leq \mathcal{K}]$ for all $t \in \mathbb{Z}_{+}$. As $\{q(t): t \geq 0\}$ is irreducible and positive recurrent by assumption and $\{\tilde{q}(t): t \geq 0\}$ has only one irreducible and positive recurrent class by construction, thus the stationary distribution exists for both $q$ and $\tilde{q}$. Thus, we have

$$
\mathbb{P}[|q(\infty)| \leq \mathcal{K}] \leq \mathbb{P}[|\tilde{q}(\infty)| \leq \mathcal{K}] \forall \mathcal{K} \in \mathbb{R} \Rightarrow \mathbb{E}[|q(\infty)|] \geq \mathbb{E}[|\tilde{q}(\infty)|]
$$

where $q(\infty)$ and $\tilde{q}(\infty)$ are random variables with distribution same as the stationary distribution of $\{q(t): t \geq 0\}$ and $\{\tilde{q}(t): t \geq 0\}$ respectively. It is easy to see that $\mathbb{E}[|\tilde{q}(\infty)|]=\left\lceil\eta^{\alpha}\right\rceil / 2$. Thus, we have

$$
\mathbb{E}\left[\mid q^{(1)}(\infty)+q^{(2)}(\infty)\right] \stackrel{*}{=} \mathbb{E}[|q(\infty)|] \geq \mathbb{E}[|\tilde{q}(\infty)|] \geq \frac{\eta^{\alpha}}{2}
$$

Also, by (14), the two-price policy satisfies Condition 1 (a) and (c), Thus, by Lemma 4, we have

$$
\mathbb{E}[|q(\infty)|] \stackrel{*}{=} \mathbb{E}\left[q^{(1)}(\infty)+q^{(2)}(\infty)\right] \geq \frac{\eta^{1-\beta} \lambda^{*}}{2(\theta+\phi)}-0.5
$$

where $\left(q^{(1)}(\infty), q^{(2)}(\infty)\right)$ denote random variable with distribution same as the stationary distribution of $\left\{q^{(1)}(t), q^{(2)}(t): t \geq 0\right\}$. Now, $(*)$ follows as $|q(t)|=q^{(1)}(t)+q^{(2)}(t)$ sample path wise as $\min \left\{q^{(1)}(t), q^{(2)}(t)\right\}=0$. Thus, we have (60).

Now, we will lower bound $\mathbb{P}\left[|q| \geq \eta^{\alpha}\right]$ for a given two-price policy. First, the stationary distribution under a given two-price policy denoted by $\left\{\pi_{i}\right\}_{i \in \mathbb{Z}}$ is given by

$$
\pi_{k}= \begin{cases}\pi_{0} & \forall k \leq\left\lceil\eta^{\alpha}\right\rceil, k \geq-\left\lceil\eta^{\alpha}\right\rceil \\ \pi_{0}\left(1-\frac{\theta \eta^{\beta}}{\eta \mu^{*}}\right)^{k-\left\lceil\eta^{\alpha}\right\rceil} & \forall k>\left\lceil\eta^{\alpha}\right\rceil \\ \pi_{0}\left(1-\frac{\phi \eta^{\beta}}{\eta \lambda^{*}}\right)^{-k+\left\lceil\eta^{\alpha}\right\rceil} & \forall k<-\left\lceil\eta^{\alpha}\right\rceil,\end{cases}
$$

where we used that $\lambda^{*}=\mu^{*}$ which follows as $\left(\lambda^{*}, \mu^{*}\right) \in \mathbb{C}$. This, gives us

$$
\begin{aligned}
\mathbb{P}\left[|q(\infty)| \geq \eta^{\alpha}\right] & =\frac{\eta^{1-\beta}\left(\mu^{*} / \theta+\lambda^{*} / \phi\right)}{\eta^{1-\beta}\left(\mu^{*} / \theta+\lambda^{*} / \phi\right)+2\left\lceil\eta^{\alpha}\right\rceil-1} \\
& \geq \frac{\left(\mu^{*} / \theta+\lambda^{*} / \phi\right)}{\left(\mu^{*} / \theta+\lambda^{*} / \phi\right)+3} \eta^{1-\alpha-\beta} \triangleq \frac{\tilde{c}_{1}}{\min \left\{\theta^{2}, \phi^{2}\right\}} \eta^{1-\alpha-\beta} \forall \eta \geq 1
\end{aligned}
$$


where $(*)$ follows as $1-\alpha-\beta<0$. Now, we have

$$
\begin{aligned}
\mathbb{E}\left[f^{2}\left(\frac{\mathbf{q}}{\eta^{\alpha}}\right)\right]+\mathbb{E}\left[g^{2}\left(\frac{\mathbf{q}}{\eta^{\alpha}}\right)\right] & =\theta^{2} \mathbb{P}\left[q^{(2)}(\infty) \geq \eta^{\alpha}\right]+\phi^{2} \mathbb{P}\left[q^{(1)}(\infty) \geq \eta^{\alpha}\right] \\
& \geq \min \left\{\theta^{2}, \phi^{2}\right\} \mathbb{P}\left[|q(\infty)| \geq \eta^{\alpha}\right] \geq \tilde{c}_{1} \eta^{1-\alpha-\beta}
\end{aligned}
$$

Thus, we have (61). Similarly, we have

$$
\begin{aligned}
\mathbb{P}\left[|q(\infty)| \geq \eta^{\alpha}\right] & \leq \frac{\eta^{1-\alpha-\beta}\left(\mu^{*} / \theta+\lambda^{*} / \phi\right)}{\eta^{1-\alpha-\beta}\left(\mu^{*} / \theta+\lambda^{*} / \phi\right)+2-\eta^{-\alpha}}\left(\text { Replace }\left\lceil\eta^{\alpha}\right\rceil \text { by } \eta^{\alpha}\right) \\
& \leq\left(\mu^{*} / \theta+\lambda^{*} / \phi\right) \eta^{1-\alpha-\beta} \triangleq \frac{\tilde{c}_{2}}{\max \left\{\theta^{3}, \phi^{3}\right\}} \eta^{1-\alpha-\beta} \forall \eta \geq 1,
\end{aligned}
$$

where $(*)$ follows as $\alpha \geq 0$ and $1-\alpha-\beta<0$. Thus, we have

$$
\begin{aligned}
\mathbb{E}\left[\left|f^{3}\left(\frac{\mathbf{q}}{\eta^{\alpha}}\right)\right|\right]+\mathbb{E}\left[\left|g^{3}\left(\frac{\mathbf{q}}{\eta^{\alpha}}\right)\right|\right] & =\theta^{3} \mathbb{P}\left[q^{(2)}(\infty) \geq \eta^{\alpha}\right]+\phi^{3} \mathbb{P}\left[q^{(1)}(\infty) \geq \eta^{\alpha}\right] \\
& \leq \max \left\{\theta^{3}, \phi^{3}\right\} \mathbb{P}\left[|q(\infty)| \geq \eta^{\alpha}\right] \leq \tilde{c}_{2} \eta^{1-\alpha-\beta}
\end{aligned}
$$

This completes the proof of the lemma.

Proof of Proposition 4 First note that if $\alpha+\beta \leq 1$, then by Theorem 3 we know that $L^{\eta} \geq$ $K \eta^{1 / 3}$. So, we will only prove Proposition 4 when $\alpha+\beta>1$. As two price policy is a special case of the general pricing policy given by (12) (13), thus, by (159), we have

$$
\begin{aligned}
L^{\eta} \geq & \eta^{2 \beta-1}\left(\frac{A}{2} \mathbb{E}\left[f^{2}\left(\frac{\mathbf{q}}{\eta^{\alpha}}\right)\right]+\frac{B}{2} \mathbb{E}\left[g^{2}\left(\frac{\mathbf{q}}{\eta^{\alpha}}\right)\right]\right) \\
& \quad-\eta^{2 \beta-1} \epsilon^{\prime \prime}\left(\mathbb{E}\left[\left|f^{3}\left(\frac{\mathbf{q}}{\eta^{\alpha}}\right)\right|\right]+\mathbb{E}\left[\left|g^{3}\left(\frac{\mathbf{q}}{\eta^{\alpha}}\right)\right|\right]\right)+s \mathbb{E}\left[\left\langle\mathbf{1}_{n+m}, \mathbf{q}\right\rangle\right] \\
\geq & \eta^{\beta-\alpha}\left(\min \{A, B\} \frac{\tilde{c}_{1}}{2}-\tilde{c}_{2} \epsilon^{\prime \prime}\right)+s \max \left\{\frac{1}{2} \eta^{\alpha}, \frac{\eta^{1-\beta} \lambda^{*}}{2(\theta+\phi)}-0.5\right\}
\end{aligned}
$$

Pick $\epsilon^{\prime \prime}=\min \{A, B\} \frac{\tilde{c}_{1}}{4 \tilde{c}_{2}}$ and optimize over all possible values of $\alpha$ and $\beta$ to get

$$
L^{\eta} \geq \inf _{\alpha \geq 0, \beta<1: \alpha+\beta>1}\left\{\eta^{\beta-\alpha} \min \{A, B\} \frac{\epsilon}{4}+s \max \left\{\frac{1}{2} \eta^{\alpha}, \frac{\eta^{1-\beta} \lambda^{*}}{2(\theta+\phi)}-0.5\right\}\right\} \stackrel{*}{=} \Omega\left(\eta^{1 / 3}\right),
$$

where $(*)$ follows as the minimum is achieved when $\beta-\alpha=\alpha=1-\beta$.

\section{K. Proof of Proposition 5}

Proof of Proposition 5 Rewriting the Bellman equation using the approximation of the bias function gives us the following optimization problem with finite variables and infinite constraints.

$$
\min _{\left(\gamma, \mathbf{b}_{l} \forall l \in[r]\right)} \gamma
$$

subject to,

$$
\gamma \geq \mathcal{R}(\mathbf{q}, \mathbf{z})+c \mathbb{E}_{\mathbf{q}, \mathbf{z}}\left[\sum_{l=1}^{r}\left\langle\mathbf{b}_{l}, V\left(\mathbf{q}^{l}, \mathbf{z}\right)\right\rangle\right]-c \sum_{l=1}^{r}\left\langle\mathbf{b}_{l}, \mathbf{q}^{l}\right\rangle \forall \mathbf{q}, \mathbf{z} \in S \times Z(\mathbf{q}),
$$


The decision variables in the above optimization problem are $g$ and $\mathbf{b}_{l} \forall l \in[r]$. By approximation, we have projected our original space of variables $\mathbf{h} \in \mathbb{R}_{+}^{b^{m+n}}$ to a lower dimensional space $\mathbf{b} \in \mathbb{R}_{+}^{(m+n) \times r}$. Let the optimal solution to the above optimization problem be $\left(\mathbf{b}_{l}^{*} \forall l \in[r], g^{*}\right)$. Now define

$$
h(\mathbf{q})=\sum_{l=1}^{r}\left\langle\mathbf{b}_{l}^{*}, \mathbf{q}^{l}\right\rangle \forall \mathbf{q} \in S .
$$

The above defined $h(\mathbf{q})$ along with $\gamma^{*}$ will be a feasible solution to the optimization problem (16) (17). Thus, the optimal value of (16) (17) will be less than or equal to $\gamma^{*}$. Thus, the proposition follows. 